LA-7656-MS

Informal Report
DR. 2349

Toward Assessing the Geothermal Potential of the

Jemez Mountains Volcanic Complex:

A Telluric-Magnetotelluric Survey

to

$\frac{7}{\omega}$

更

$\stackrel{\text { L }}{\mathrm{L}}$ 


\section{DISCLAIMER}

This report was prepared as an account of work sponsored by an agency of the United States Government. Neither the United States Government nor any agency Thereof, nor any of their employees, makes any warranty, express or implied, or assumes any legal liability or responsibility for the accuracy, completeness, or usefulness of any information, apparatus, product, or process disclosed, or represents that its use would not infringe privately owned rights. Reference herein to any specific commercial product, process, or service by trade name, trademark, manufacturer, or otherwise does not necessarily constitute or imply its endorsement, recommendation, or favoring by the United States Government or any agency thereof. The views and opinions of authors expressed herein do not necessarily state or reflect those of the United States Government or any agency thereof. 


\section{DISCLAIMER}

Portions of this document may be illegible in electronic image products. Images are produced from the best available original document. 
This work was supported by the US Department of Energy, Geothermal Energy Division.

This report was prepared as an account of work sponsored by the United States Government. Neither the United States nor the United States Department of Energy, nor any of their employees, nor any of their contractors, subcontractors, or their employees, makes any warranty. express or implied, or assumes any legal liability or responsibility for the accuracy. completeness, or usefulness of any information, apparatus, product, or process disclosed, or represents that its use would not infringe privately ow ned rights. 
LA-7656-MS

Informal Report

UC-66a

Issued: February 1979

\title{
Toward Assessing the Geothermal Potential of the Jemez Mountains Volcanic Complex: A Telluric-Magnetotelluric Survey
}

\author{
John F. Hermance*
}

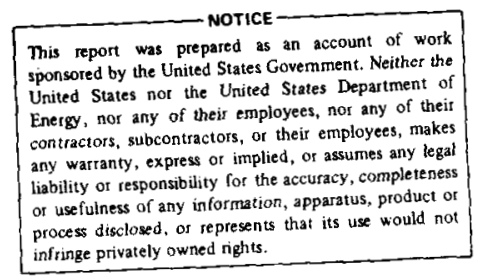

"Brown University, Providence, RI 02912

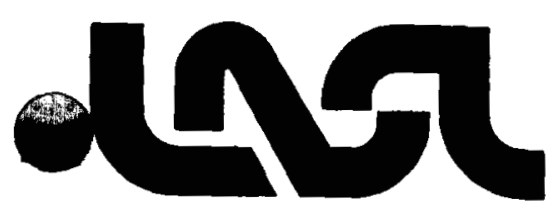


TOWARD ASSESSING THE GEOTHERMAL FOTENTIAL OF THE

JEMEZ MOUNTAINS VOLCANIC COMPLEX: A TELLURIC-MAGNETOTELLURIC SURVEY

\author{
by
}

John F. Hermance

Brown University

\begin{abstract}
Telluric-magnetotelluric studies were performed in the Jemez Mountains of north-central New Mexico to characterize the total geothermal system of the Valles Caldera and to be integrated with an east-west regional survey supported by the United States Geological Survey. The data from the regional survey indicate that electrically the San Juan Basin to the west of the Jemez Mountains is rather homogeneous in contrast to the eastern side near Las Vegas where the presence of a broad heterogeneous structure is clearly sensed. The data from the Jemez Mountain area are strikingly similar to other Rio Grande rift data and suggest a conducting layer at a depth of approximately $15 \mathrm{~km}$. The telluric data indicate that the hydrothermal system in the area is of a localized nature.
\end{abstract}

\title{
I. INTRODUCTION
}

The central objective of this telluric-magnetotelluric research frogram funded by the LC.S Alamos Scientific Laboratory (LASL) was to provide constraints on the characterization of the total geothermal system associated with the evolution of the Jemez Mountains in north-central New Mexico and was closely integrated with a regional survey initiated by our group and supported by the United States Geological Survey (USGS).

For those sites where reasonable data were available, conventional magnetotelluric parameters were calculated leading to standard estimates of the tensor impedance, which are included as an appendix to this report. Moreover, in many cases, two sites were operated simultaneously and telluric transfer tensors were calculated as well.

For those sites that exhibit a consistent two-dimensional quality, models of interpreted resistivity versus depth were calculated using a "continuous" inversion scheme for the resistivity determined from the electric field polarized parallel to geologic strike. In some cases, data from several sites close to each other can in fact be overlaid and lead to consistent, though somewhat generalized models of the actual resistivity as a function of depth. 
A. The Regional Survey

Maps of north-central New Mexico on which normalized telluric ellipses at each site are shown represent the local polarization of the horizontal electric field when referred to a circularly polarized electric field of unit amplitude.

The telluric ellipse at a site on the Colorado Plateau near Farmington has practically a circularly polarized telluric field. Apparently, this site situated in the broad San Juan Basin is not affected by local inhomogeneities. In contrast, sites on the eastern side of the Sangre de Cristo Mountains near Las Vegas are clearly sensing the presence of a broad heterogeneous structure. Telluric ellipses at sites located in the Rio Grande rift near Santa Fe have similar qualities to those from some sites in the Jemez Mountains (JE-B, Baca-4, $\mathrm{JE}-10$, JE-11). Moreover, broadband magnetotelluric data from these sites are remarkably similar.

B. The Baca Grant Survey

Through the cooperation of the Geothermal Division of Union $0 i 1$ and personnel at LASL, seventeen short-period (10-100 s) telluric sites were occupied in the Baca Grant within the Valles Caldera. Maps have been plotted showing rormalized telluric ellipses calculated at 20 seconds for these sites. Anomalously large telluric ellipses at two sites in Valle Grande (southeastern part of the caldera) apparently represent a local perturbation due to a resistive resurgent dome. In sharp contrast, sites in the north-western part of the caldera have anomalously low amplitudes which may be associated with locally enhanced electrical conductivities in the shallow crust as a result of local hydrothermal activity.

On the other hand, one is impressed with the similarity of the telluric field polarization at many sites. In general, the telluric field pattern allows one to discriminate between those sites representative of regional features and those sites influenced by local perturbations.

C. Deep Structure of the Jemez Mountains

Magnetotelluric data from the Jemez base site has an inflection in the magnetotelluric response between 100 and 1000 seconds strikingly similar to the data from the southern Rio Grande rift reported by Pedersen and Hermance 1 and the north-central rift reported by Hermance and Pedersen. 2 The Jemez data suggest the presence of a conducting layer at a depth of approximately $15 \mathrm{~km}$ beneath the Jemez Mountains.

Magnetotelluric data recorded at a number of satellite sites in the Jemez Mountains show a similar feature and suggest that the conducting layer, present beneath the Rio Grande rift and perhaps representing a magma accumulation, extends laterally beneath the Jemez Mountains and the Valles Caldera.

Perhaps the resurgence of volcanic activity in the Valles Caldera several million years ago was associated with the creation of this zone at that time. Since then, the thermal imprint of this event has declined in intensity at shallow levels, al though molten magma, probably of a tholeiitic composition, still persists at $15 \mathrm{~km}$ depth. The telluric data in the Jemez Mountains do not suggest a major conducting anomaly at shallow depth such as might be associated with a major magma body or even a widespread hydrothermal system. At present, significant concentrations of crustal hydrothermal activity appear to be localized in nature and controlled more by shallow structure than by a regional convective system associated with an active magma body. 


\section{OVERVIEW OF THE F.ESEARCH PROGRAM}

The central objective of the telluric-magnetotelluric research program described below was to provide constraints on the characterization of the total geothermal system associated with the evolution of the Jemez Mountains in rorthcentral New Mexico. Clearly, the most vigorous geothermal systems are those for which the hydrothermal, the hot dry rock, and the magmatic source rock are intimately coupled to one other. Therefore, our goal was to assist in evaluating the coupling of these three geothermal regimes beneath this area.

This research was closely integrated with a parallel program of our group supported by the USGS. This involved a regional telluric-magnetotelluric traverse beginning in the Great Plains, crossing the Rio Grande rift in the vicinity of the Jemez Mountains and terminating on the Colorado Plateau. The two research programs were designed to complement each other in both a practical and a philosophical way. In a fractical sense, assembling equipment, performing the field programs, reducing the data, and interpreting the data were optimized in that redundant activities and costs were minimized.

In a philosophical sense, the USGS program provided regional constraints on thermal processes in the deep crust and upper mantle, whereas the LASL program was a site-specific characterization of not only the geothermal potential of the Jemez Mountains and the Valles Caldera, but the relation of this geothermal activity to deeper processes in the crust and mantle as well.

Upon completion of the survey the sites shown in Table I had been cccupied. However, good data were not retrieved from all the sites. This was due to a number of factors, such as local lightning storms destroying data quality, the inability to transfer data from tapes, and in some cases inexperience cn the part of the field crew in discriminating between good and bad data sets while on-site.

However, for those sites where reasonable data were available, conventional magnetotelluric parameters were calculated leading to standard estimates of the tensor impedance 3,4 for computer codes. 5 Moreover, in many cases, two sites were operated simultaneously and telluric transfer tensors were calculated as well 6,7

Attached as an appendix to this report are the various telluric and magnetotelluric parameters for each site. In particular, principal resistivity values as a function of period are tabulated and plotted in graph form.

For those sites that exhibit a consistent two-dimensional quality, models of true resistivity versus depth were calculated using the inversion scheme of Francis Bostick 3 for the resistivity determined from the electric field polarized parallel to geologic strike. As is well-known, apparent resistivities determined from this component (as opposed to the perpendicular component) are the least affected by near-surface structural heterogeneities. Often the parallel apparent resistivity leads to reasonable estimates of the regional vertical electrical structure. In our case, data from several sites close to each other can in fact be overlaid and lead to consistent, though somewhat generalized models of the actual resistivity as a function of depth. On the other hand, a number of data sets show the contaminating effects of geologic structure local. to the specific site; hence these data cannot be directly used in delineating the lateral character of deep coherent structures within a given area. Nevertheless, our study has suggested the results summarized in the following section. 
TABLE I

TELLURIC-MAGNETOTELLURIC SITES OCCUPIED BY BROWN UNIVERSITY IN NORTH-CENTRAL NEW MEXICO

\begin{tabular}{ll}
\multicolumn{1}{c}{ AREA } & TYPE OF SITE \\
\hline Farmington & 2 base \\
& \\
& \\
Jemez Mtns. & 12 base \\
& 17 remote \\
& (Baca Grant) \\
& 1 base \\
Las Vegas, NM & 2 satellites \\
& 1 base \\
Santa Fe & 2 satellites \\
& 2 remote
\end{tabular}

PERIOD RANGE

10-10000 s

w/short period

data from indus-

trial source

$10-10000 \mathrm{~s}$

$10-4000 \mathrm{~s}$

17 remote

$10-10000 \mathrm{~s}$

$10-3000 \mathrm{~s}$

$10-10000 \mathrm{~s}$

$10-4000 \mathrm{~s}$

$10-100 \mathrm{~s}$

\section{SCIENTIFIC RESULTS}

A. The Regional Survey

Included in this report is a map (Fig. 1) of north-central New Mexico on which our telluric-magnetotelluric sites are located. At each site a normalized telluric ellipse is shown, which represents the local polarization of the horizontal electric field, when referred to a circularly polarized electric field of unit amplitude induced in a homogeneous earth having a resistivity of $20 \mathrm{ohm}-\mathrm{m}$ (Hermance and Pedersen, manuscript in preparation). For this presentation, we assume that local distortion of the telluric field is of surficial origin so that the telluric transfer matrix has only real elements. The advantage of presenting data in this way is that one circumvents the question of two-dimensionality or three-dimensionality of geologic structures at a field site. The telluric ellipses represent basic information concerning the distortion of regional telluric fields above and beyond any consideration of two or three dimensionality.

For example, site $\mathrm{F}-2$, on the Colorado Plateau near Farmington has an almost circularly polarized telluric field. Apparently, this site situated in the broad San Juan Basin is not affected by local inhomogeneities. In contrast, the sites LV-Base and LV-2 on the eastern side of the Sangre de Cristo Mountains are clearly sensing the presence of a broad heterogeneous structure.

Other heterogeneous features appear on the map. For example, in the Jemez Mountains, JE-6 shows a strong east-west polarization, whereas JE-5, although nearby, has a relatively uniform polarization. Such an effect could be explained by JE- 6 being located above a basement horst block, whereas JE-5 is located a.t the edge of the block. 


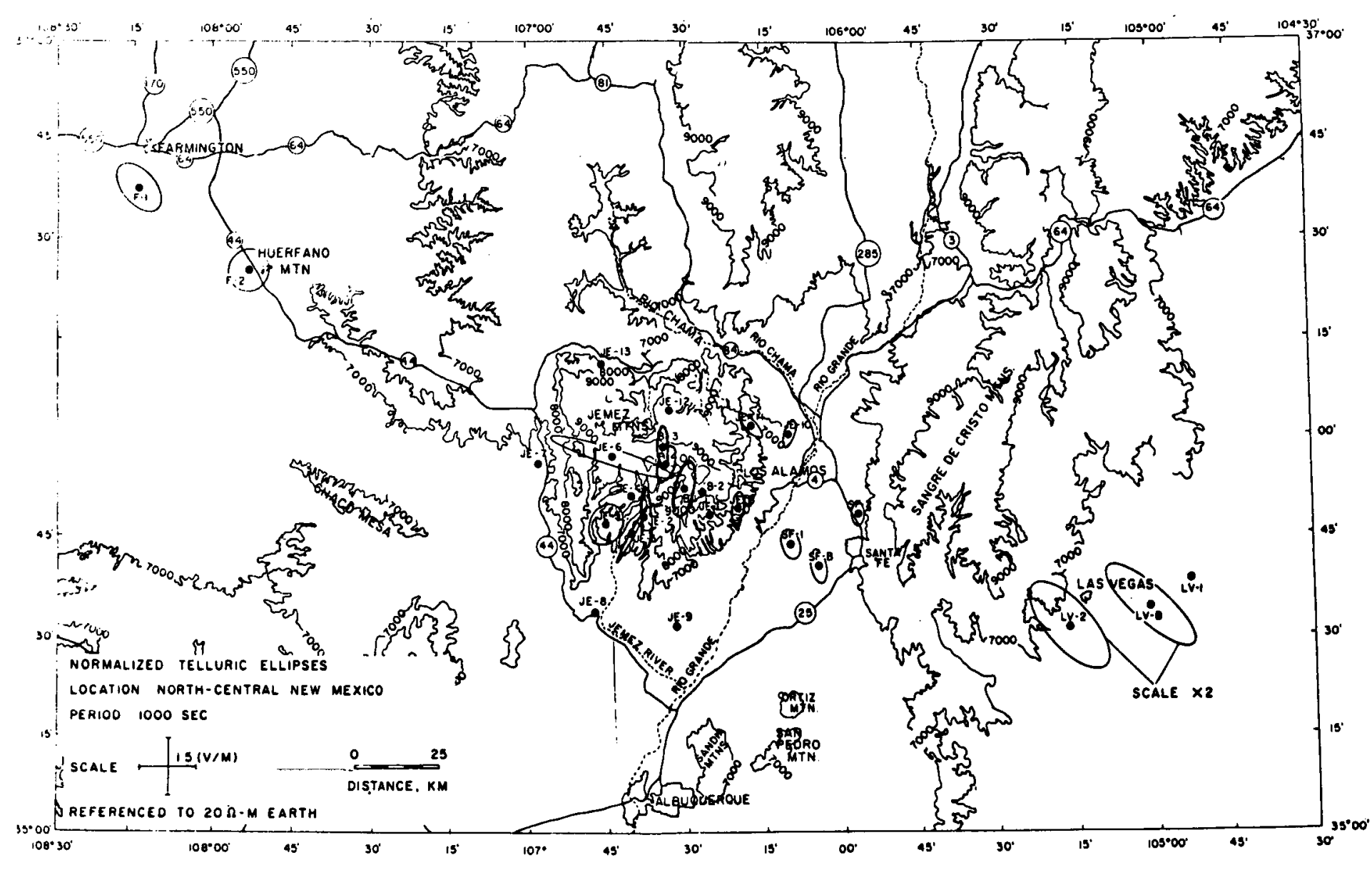

Fig. 1.

Normalized telluric ellipses - North Central New Mexico. 
Sites JE-2 and JE-3 were located on top of a sharp-walled mesa and apparently are disturbed by local topographic effects.

In the Baca Grant, B- 1 is decidedly larger that JE-Base, while B-3 is only moderately enhanced. $B-1$ is apparently seeing a local perturbation due to the resurgent dome on whose flank it is located.

We feel that it is significant that the telluric ellipses at sites located in the Rio Grande rift have similar qualities to those in the Jemez Mountains. We villl see that the broadband magnetotelluric data from these six sites (seven, including $B-3$ ) are remarkably similar.

B. The Baca Grant Survey

Through the cooperation of the Geothermal Division of Union $0 i 1$ and personnel at LASL, seventeen short-period (10-100 s) telluric sites were occupied in the Baca Grant within the Valles Caldera. Included as part of this report is a map (Fig. 2) showing normalized telluric ellipses calculated at $20 \mathrm{~s}$ for these sites.

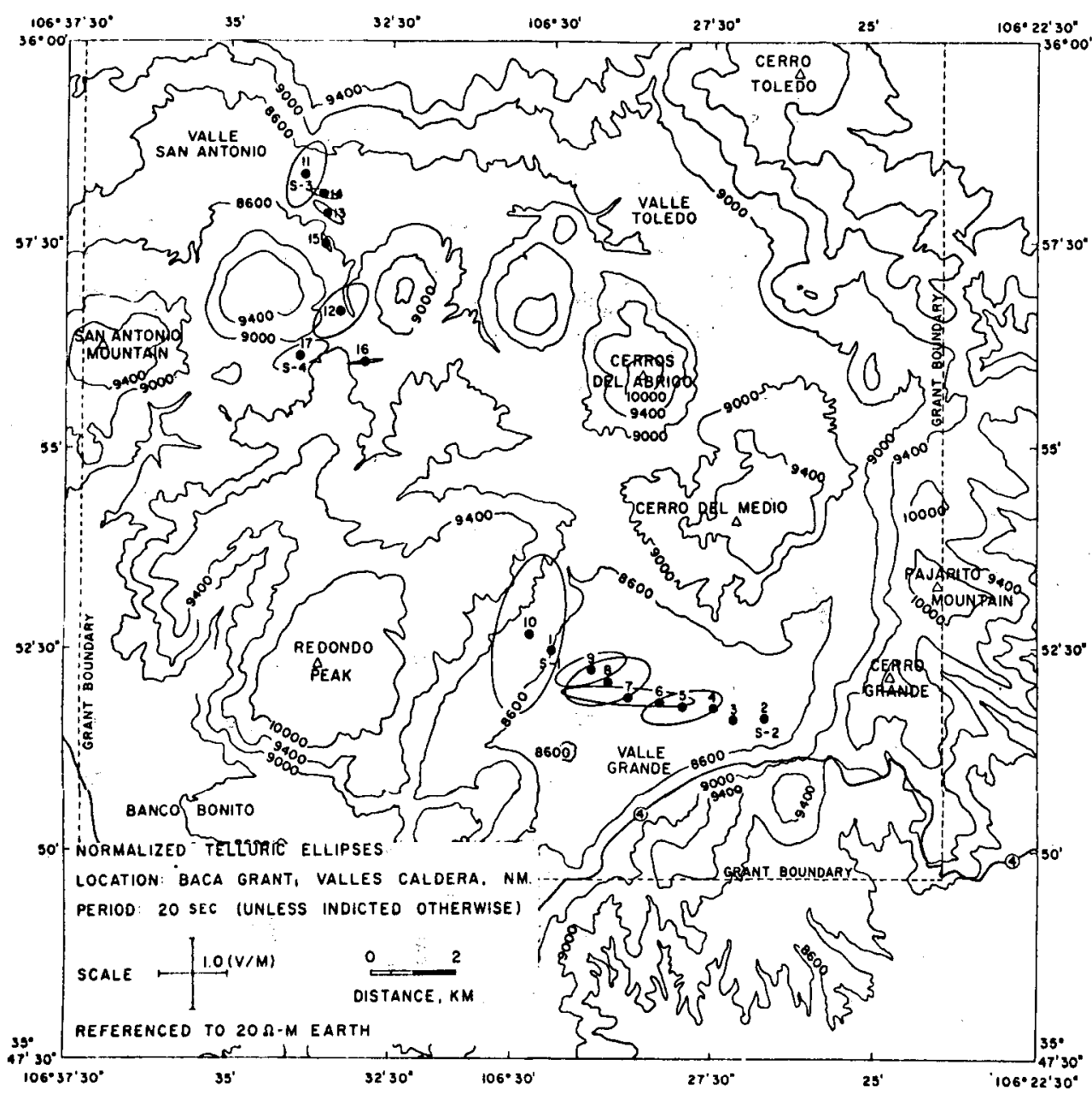

Fig. 2.

Normalized telluric ellipses - Baca Grant. 
Inspection of the map reveals anomalously large telluric ellipses at sites 1 and 10, apparently representing a local perturbation due to the resurgent dome. In sharp contrast, sites 13-16 have anomalously low amplitudes. However, one must be cautioned that sites 14-16 also have low telluric field predictabilities (on the order of 0.3 ) whereas site 13 has predictabilities on the order of 0.75 . Although we have not evaluated any possible bias in the telluric transfer tensor due to these low predictabilities, we nevertheless feel that these four sites (13-16) may indeed be associated with locally enhanced electrical conductivities in the shallow crust,perhaps a result of local hydrothermal activity. Clearly, a more detailed survey should be considered for this area.

A somewhat different impression emerges when the ellipses at sites $5,8,9$, 11 , $(B-3), 12$, and $17(B-5)$ are compared. Here one is impressed with the similarity of the telluric field polarization of these sites.

In general, we feel the telluric field pattern allows one to discriminate between those sites representative of regional features and those sites influenced by local perturbations.

C. Deep Structure of the Jemez Mountains

Magnetotelluric data from the Jemez base site (JE-B) are presented in Fig. 3, where data are shown in which the electric field predictability for either component is greater than 0.8 . In Fig. 4 , we show data where both predictabilities are greater than 0.9 . This appears to be a very restrictive condition.

In Fig. 5, we show data where either predictability (usually E-Max) has to be larger than 0.9 and where the skewness coefficients are less than 0.4 . The circled points represent data for which the maximum electric field component has a predictability less than $0.85(0.82$ to 0.84$)$. These data may be biased downward somewhat from their true values by noise on the magnetic component. Nevertheless, the inflection in the magnetotelluric response between 100 and $1000 \mathrm{~s}$ is strikingly similar to the data from the southern Rio Grande rift reported by Pedersen and Hermance ${ }^{1}$ and the north-central $r i f t$ reported by Hermance and Pedersen ${ }^{2}$.

The interpretation of magnetotelluric data from those two areas indicated a zone of melt accumulation at a depth of $15 \mathrm{~km}$ beneath the north-central rift (Santa Fe) and at a depth of $27 \mathrm{~km}$ beneath the southern rift (El Paso). Since the best-resolved parameter of this layer was its section-conductance (thickness-conductivity product), a layer $750 \mathrm{~m}$ thick consisting of $100 \% \mathrm{melt}$ $\left(\mathrm{T}=1200^{\circ} \mathrm{C} ; \quad=2 \mathrm{~S} / \mathrm{m}\right)$ is an upper limit on the melt concentration, whereas a layer $6 \mathrm{~km}$ thick consisting of $15 \%$ melt is a lower limit on the melt concentration.

It is instructive to consider the data from the Jemez base site in the light of these other results. In Fig. 6 , we show a range of curves which might fit the principal apparent resistivity data parallel to geologic strike. Using Francis Bostick's continuous inversion scheme 3 , we have considered six models that fall within this range and have the responses indicated in Figs. 7 and 8 . The interpreted resistivity in ohm-m as a function of depth in kilometers for each model is shown is Figs. 9-14, with a composite of all models shown in Fig. 15.

The degree to which apparent resistivity data in the period range 100-1000 s is biased downward by magnetic noise significantly affects the interpretation.

One does however obtain the impression from both the magnetotelluric data in Fig. 5 as well as the composite of the interpretated resistivities in Fig. 15, that the data do suggest the presence of a conducting layer at a depth of approximately $15 \mathrm{~km}$ beneath the Jemez Mountains. 


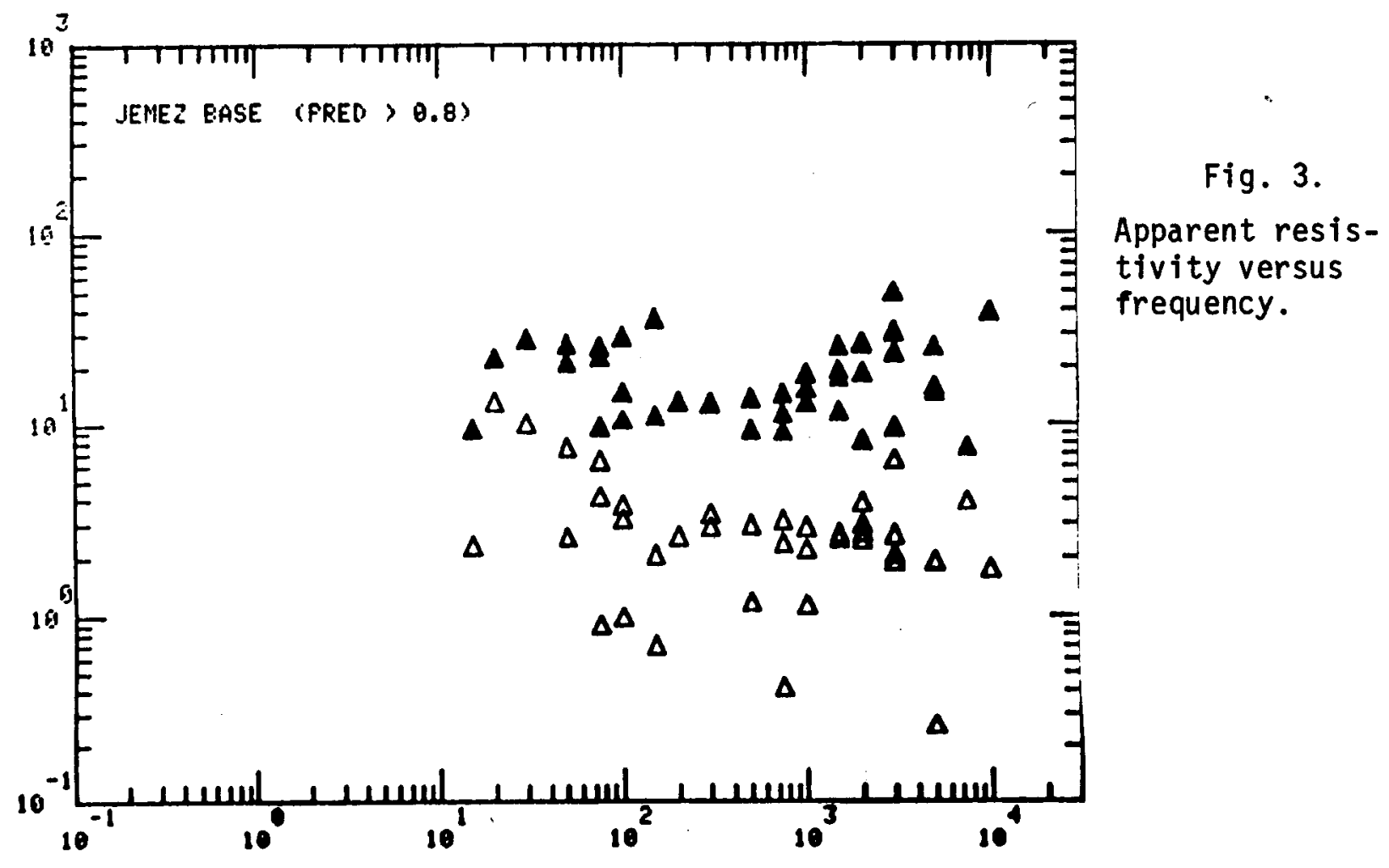

Fig. 4.

Apparent resistivity versus frequency.

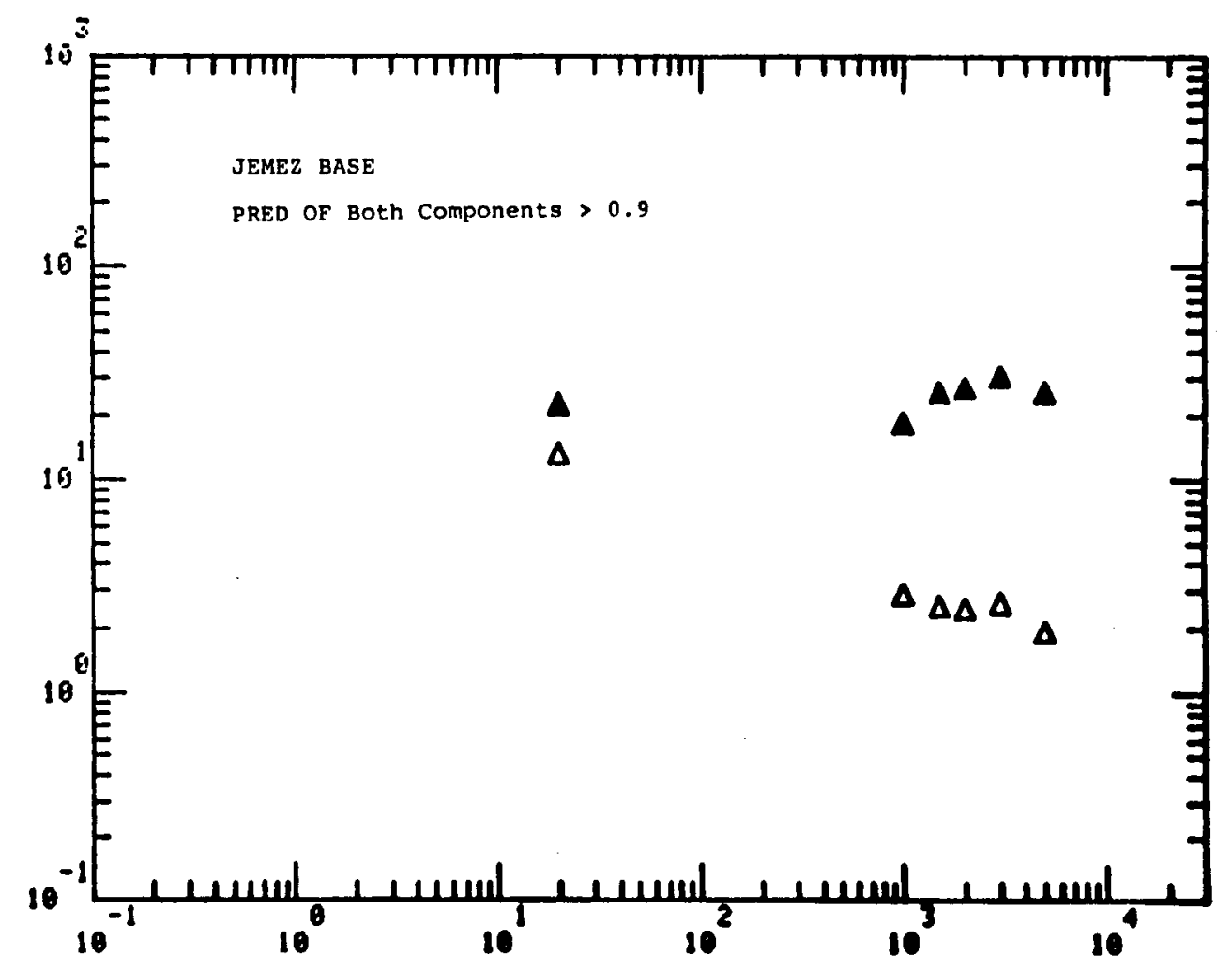



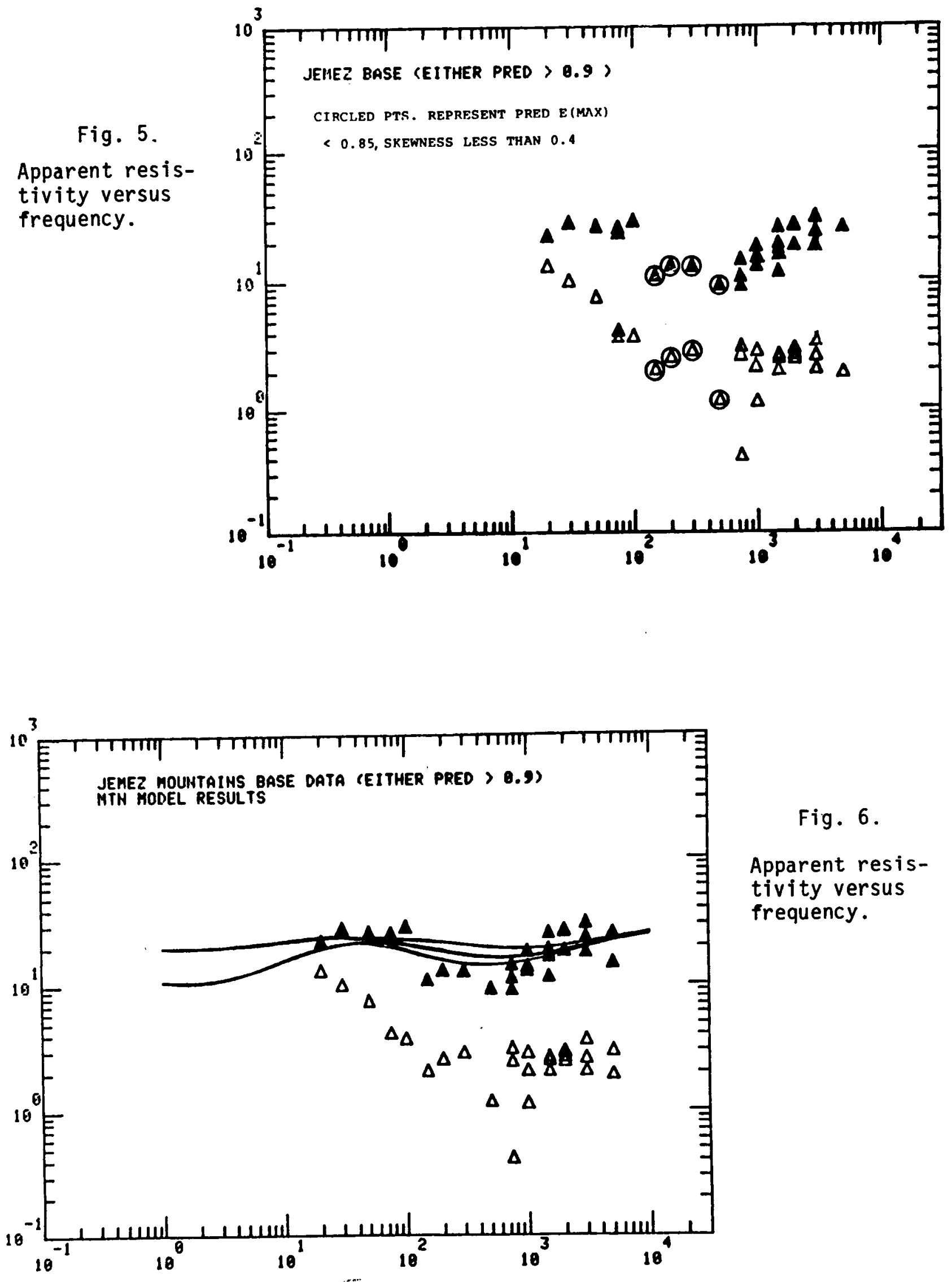

Fig. 6 .

Apparent resistivity versus frequency. 

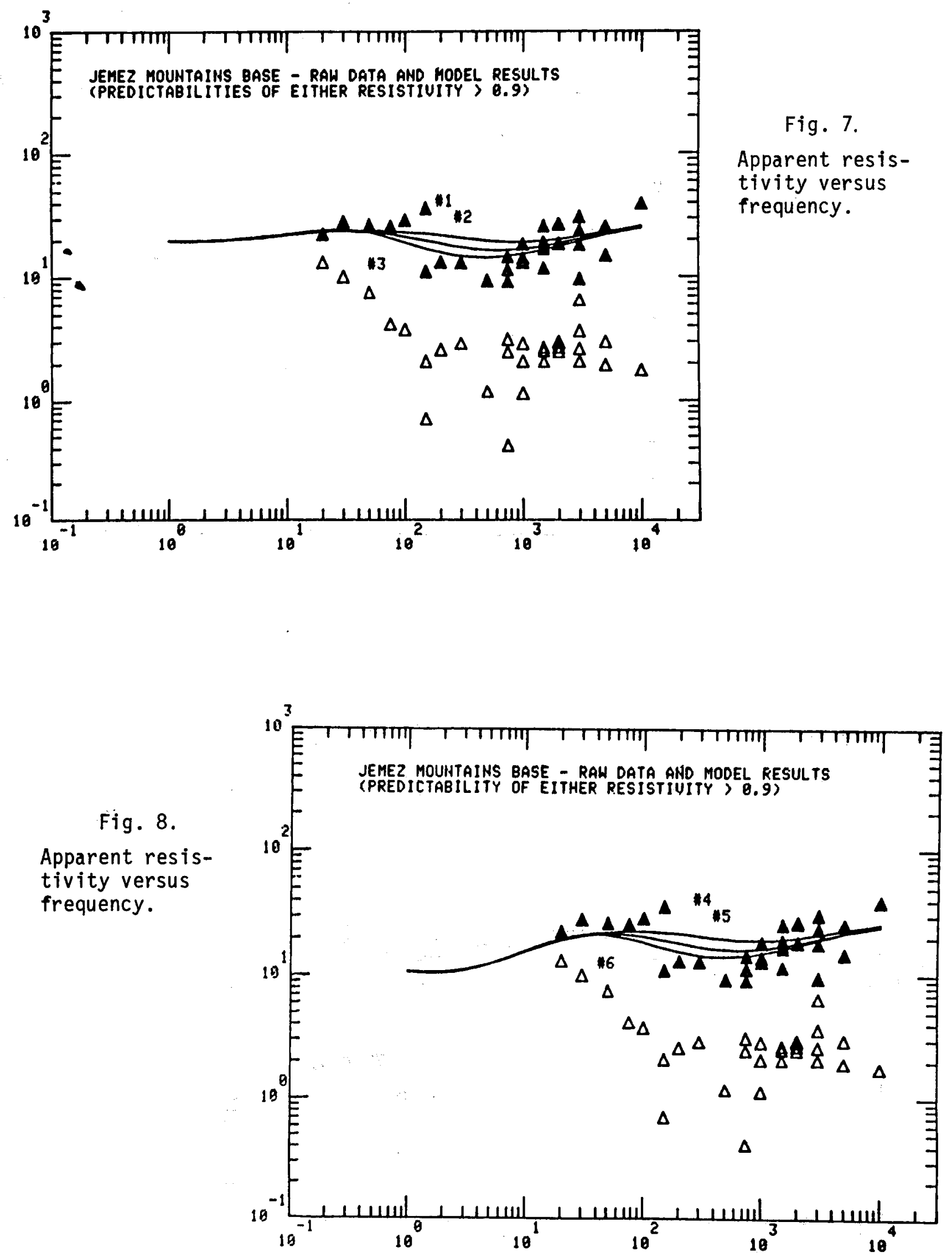

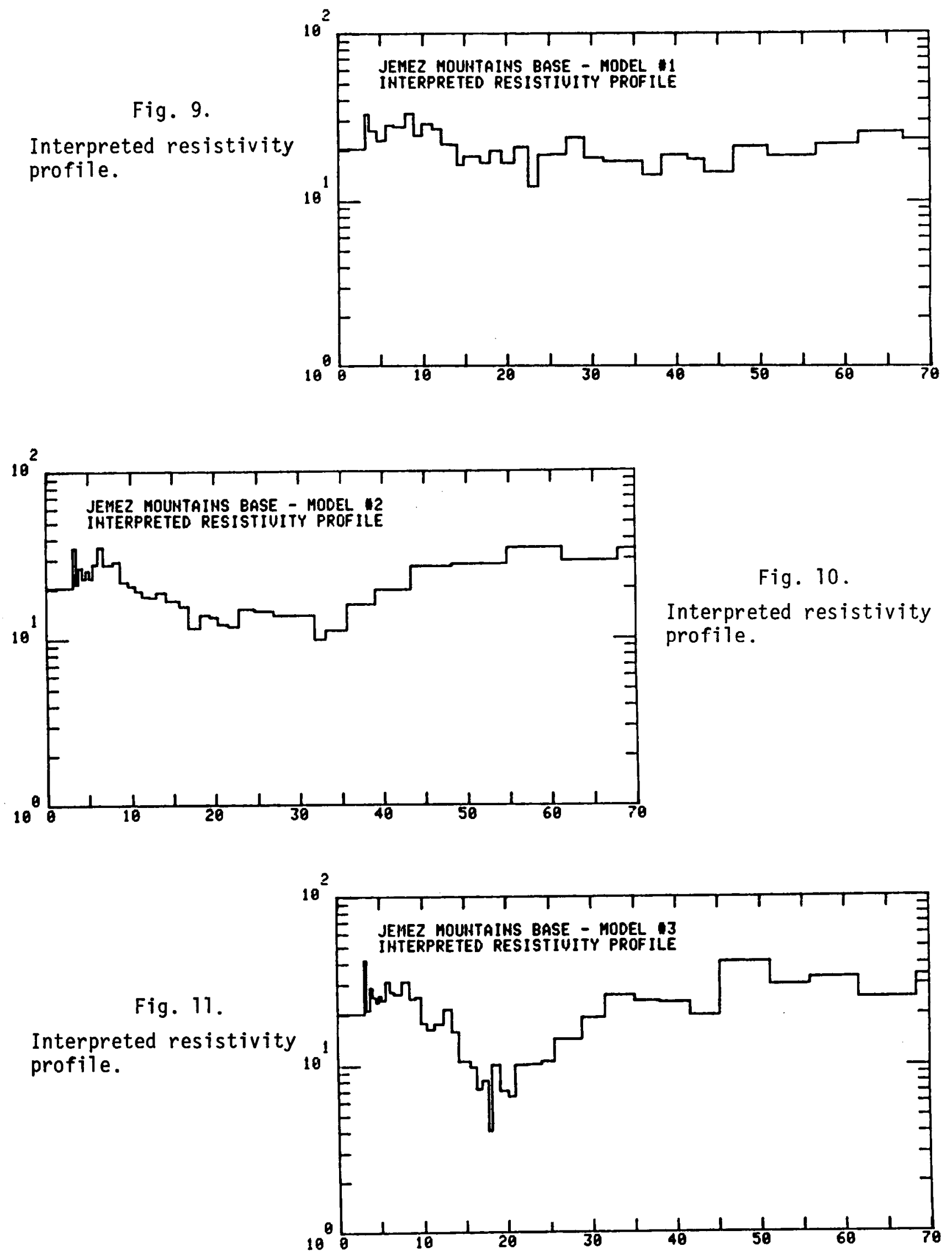


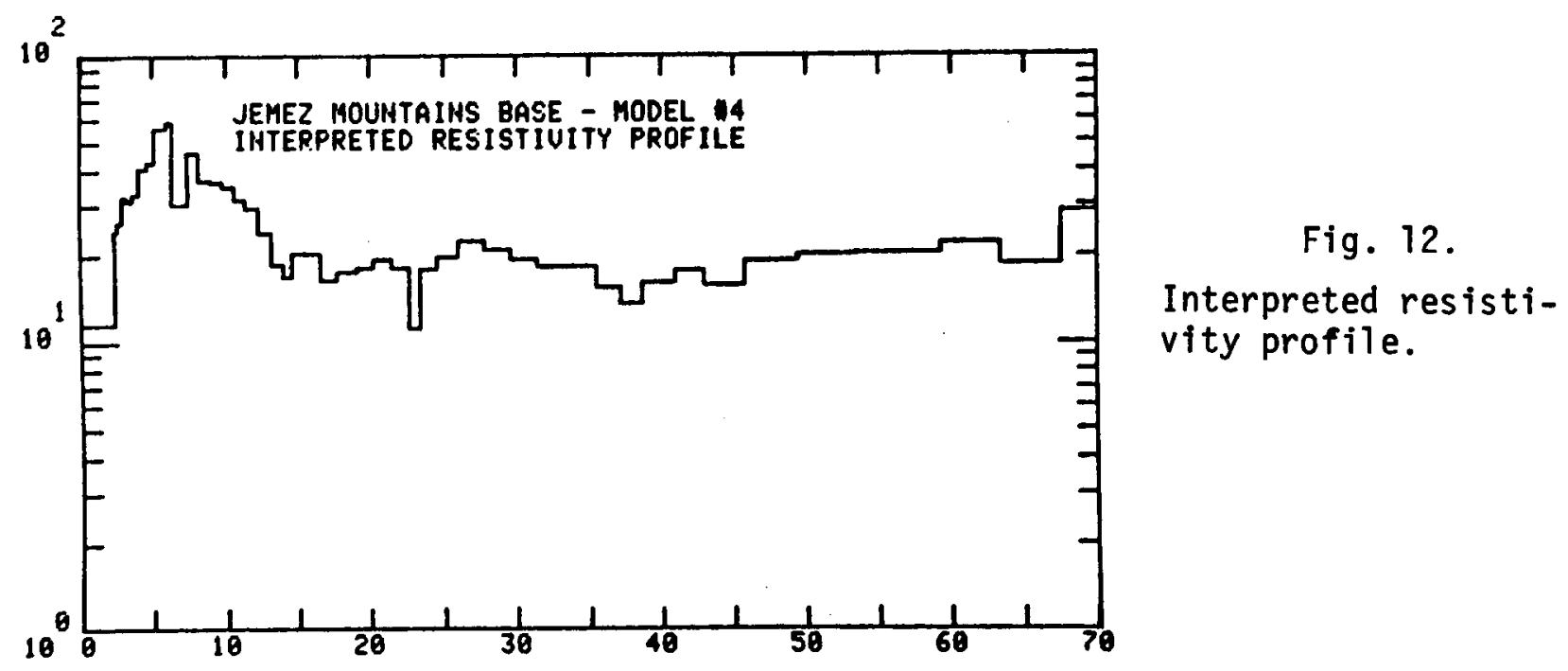

Fig. 13.

Interpreted resistivity profile.
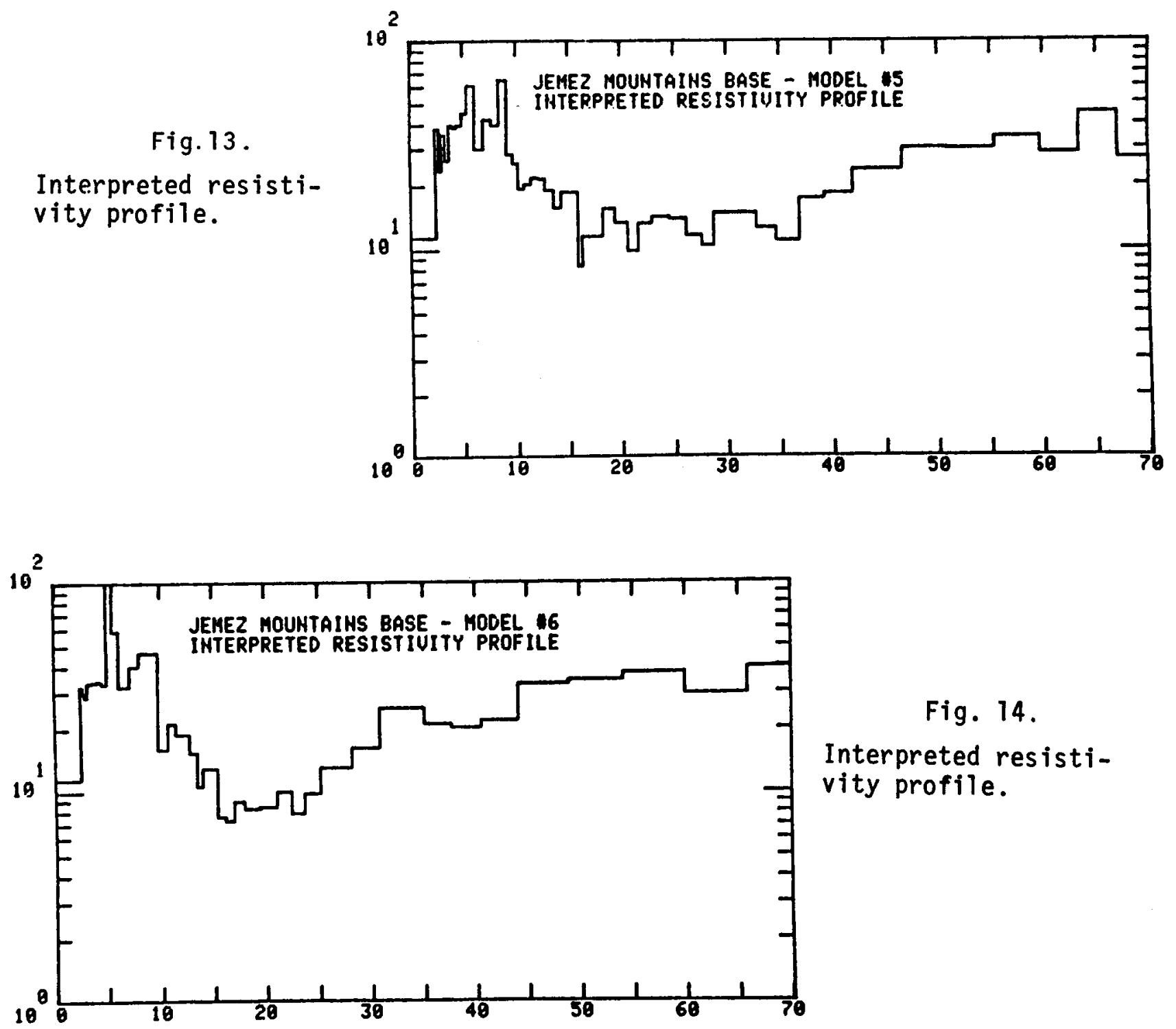

Fig. 14 .

Interpreted resistivity profile. 


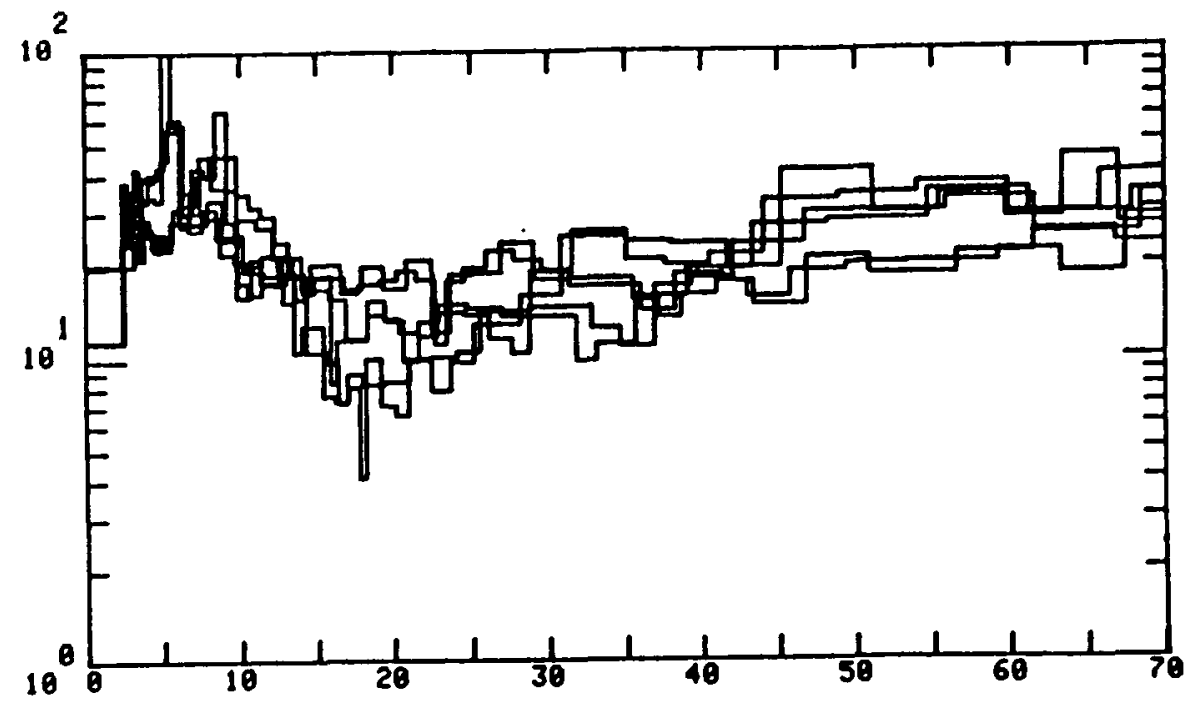

Fig. 15.

Interpreted resistivity profile.

In Figs. 16-17, the responses of Models 1-6 are compared with magnetotelluric data recorded at a number of satellite sites in the Jemez Mountains. At this point we feel that the similarities tetween these sites dominate over the differences and that the conducting layer, present beneath the Rio Grande rift and perhaps representing a magma accumulation, extends laterally beneath the Jemez Mountains and the Valles Caldera.

We might speculate that the resurgence of volcanic activity in the Valles Caldera several million years ago was associated with the creation of this zone at that time. Since then, the thermal imprint of this event has declined in intensity at shallow levels, al though mol ten magma still persists at $15 \mathrm{~km}$.

The telluric data in the Jemez Mountains do not suggest a major conducting anomaly at shallow depth such as might be associated with a major magma body or even a widespread hydrothermal system. Al though present manifestations of geothermal activity in the Valles Caldera are ultimately related to volcanism, we see the present activity as a product of much more intensive activity over the last several million years. At present, significant concentrations of crustal hydrothermal activity appear to be localized in nature and controlled more by shallow structure than by a regional convective system associated with an active magma body.

\section{ACKNOWLEDGMENTS}

We would like to acknowledge the extraordinary assistance provided by personnel at LASL, particularly Paul Kintzinger, Tom Shankland, Francis West, and John Rowley. Also, our work on the Baca Grant in the Valles Caldera would not have teen possible vithout the permission of the Geothermal Division of Union $0 i 1$ and the kind assistance of Dick Dondanville and Alan Lattanner. Finally we'd like to thank Pat Dunigan for allowing access to his ranch. 

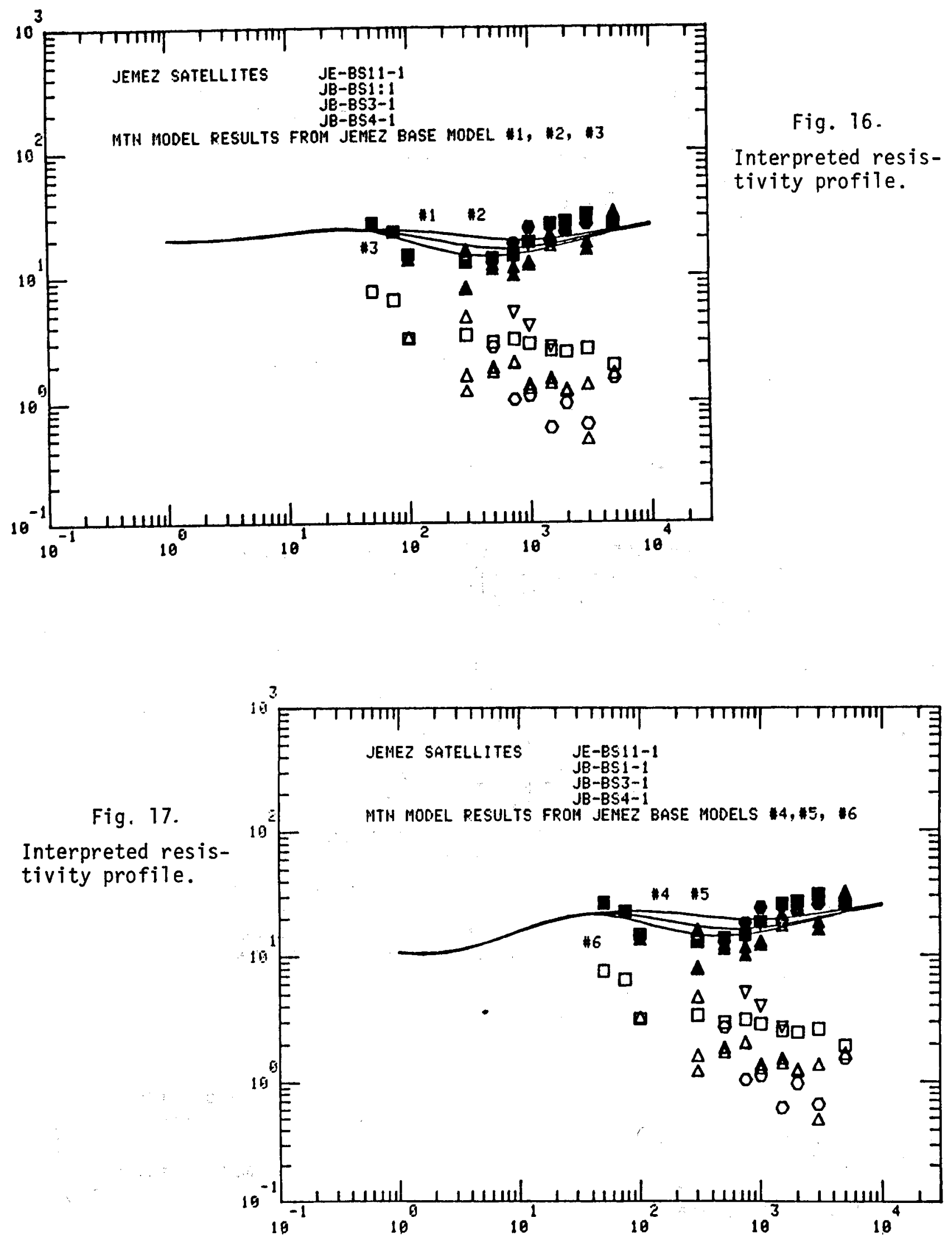


\section{REFERENCES}

1. J. Pedersen and J. F. Hermance, "Towards Resolving the Absence or Presence of an Active Magma Chamber Under the Southern Rio Grande Rift Zone", (abstract), EOS, Trans. Am. Geophy. Un. 57, 1014 (1976).

2. J. F. Hermance and J. Pedersen, "The Deep Structure of the Rio Grande Rift: A Magnetotelluric Study," (in press).

3. W. E. Sims and F. X. Bostick, Jr., "Methods cf Magnetotelluric Analysis," Elec. Res. Lab Tech. Rept. 58, 86 pp., Univ. Tex., Austin (1969).

4. K. Vozoff, "The Magnetotelluric Method in the Exploration of Sedimentary Basins," Geophys. 37, 98-141 (1972).

5. R. E. Thayer, "Telluric-Magnetotelluric Investigations of Regional Geothermal Processes in Iceland," Phd. Diss., Brown Univ., 255 pp., (1975).

6. J. F. Hermance and R. E. Thayer, "The Telluric-Magnetotelluric Method," Geophys. 40, 664-668 (1975).

7. J. F. Hermance, R. E. Thayer, and A. Bjornsson, "The Telluric-Magnetotelluric Method in the Regional Assessment of Geothermal Potential," Proc. Second U.N. Symp. on Devel. and Use of Geothermal Resources, Vol. 2, pp. 1037-1048, UG0-000-00005-1, Supt. Doc., U. S. G.P.0., Wash. DC (1976).

\section{APPENDIX \\ NORMALIZED TELLURIC ELLIPSES}

I. LOCATION - BACA GRANT: VALLES CALDERA, NEW MEXICO

These data were acquired in cooperation with the Union Geothermal Division.

Section A.

Normalized telluric ellipses for a complex telluric transfer tensor driven by a unit amplitude telluric field having clockwise and counterclockwise circular polarizations.

\section{Section B.}

Normalized telluric ellipses for a real telluric transfer tensor driven by a unit amplitude circularly polarized electric field induced in a homogeneous

medium having a resistivity of $20 \mathrm{ohm}-\mathrm{m}$.

$$
\begin{array}{ll}
\text { Part 1. } & 20 \text { seconds } \\
\text { Part 2. } & 30 \text { seconds }
\end{array}
$$


Section $C$.

Normalized and compensated telluric ellipses for Baca Satellite 1 (B-1) determined at $50 \mathrm{sec}$. and $65 \mathrm{sec}$.

II. LOCATION - REGIONAL DISTRIBUTION OF SITES ACROSS NORTHERN NEW MEXICO

These data were acquired through a joint research program supported by the Los Alamos Scientific Laboratory and the U. S. Geological Survey. The cooperation of the Union Geothermal Division was extended by way of allowing access to Union's leased properties in the Baca Grant, Valles Caldera.

The following data is arranged alphabetically by site:

$$
\begin{array}{ll}
\text { Farmington } & \text { Las Vegas } \\
\text { Jemez Mountains } & \text { Santa Fe }
\end{array}
$$

and consists of two figures from each location representing the telluric ellipse referred to a unit amplitude circularly polarized electric field induced in a homogeneous medium having a resistivity of $20 \mathrm{ohm}-\mathrm{m}$. One figure represents the transformation generated by applying only the real elements of the telluric transfer tensor, and results in a single ellipse. The other figure represents the transformation generated by applying a clockwise and counterclockwise electric field to the complex elements of the telluric transfer tensor, and results in an ellipse for both polarizations. 7

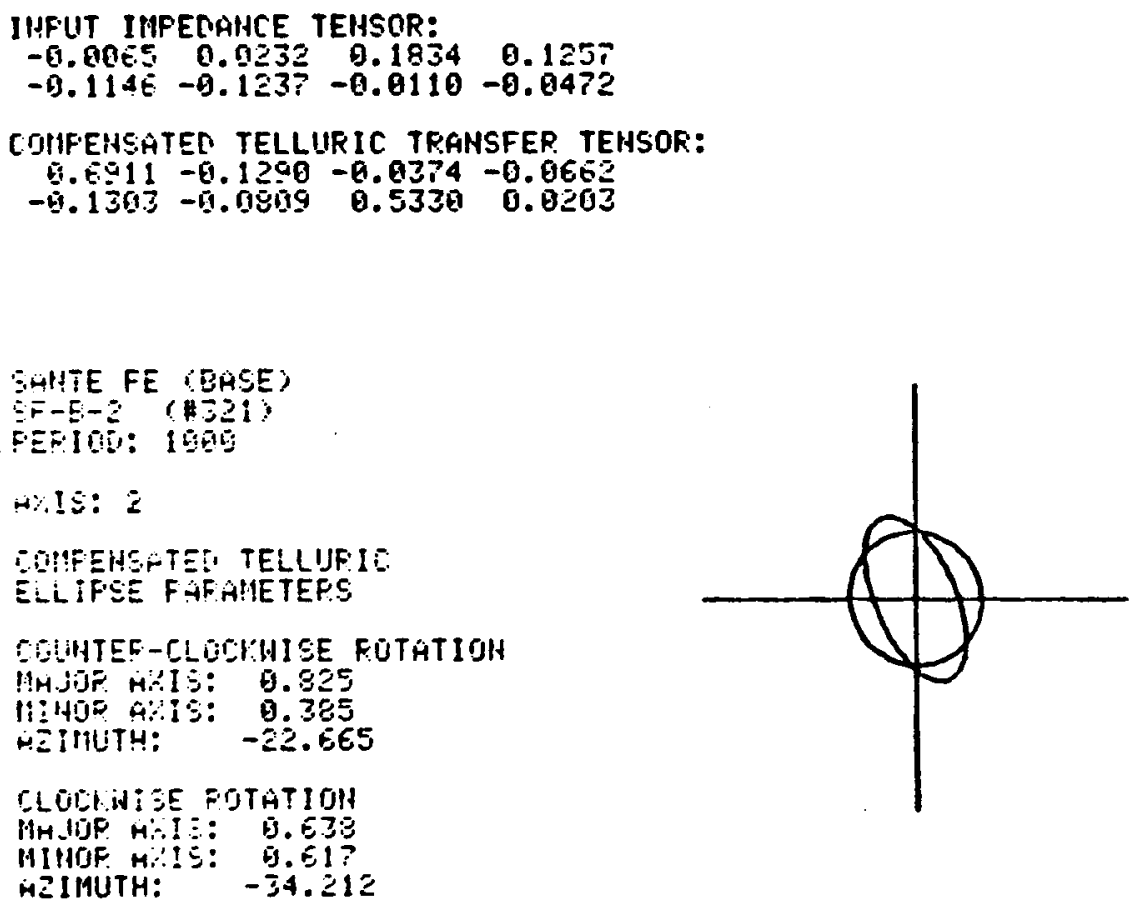


IHFUT IMPEOAHCE TEHSOR:

$\begin{array}{llll}0.0468 & 0.0871 & 0.2974 & 0.3322\end{array}$

$-6.2641-0.2386-0.0866-0.0544$

COMFEHSATET TE'LUP.IC TRAMSFER TEHSOR:

- .2006 0.2792 $-0.2992-0.9901$

FAFHIHCTOH (BASE)

FA-E-1 ( 4 LE?

FEPIOI: $16 \overline{60}$

A.!IS: ?

CORPE!SATELT TELLUPIC

ELLIFSE FAFAMETEPS

COUHTEF-CLOEKHIEE POTATIOH

LiW IOJF HIIS: 1.496

Miliof 4 EIS: 1.104

HE!HUTH: - -27.790

CLICHHISE ROTATIOH

MMJUF AOI:: 1.566

MIHOP ATIS: 9.613

A2I!IUTH: $-4 \overline{1} .341$

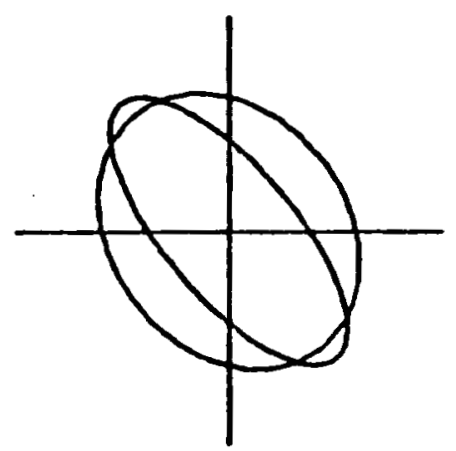

INPUT IMPEDANCE TEHSOR

$0.0465 \quad 0.0871 \quad 0.2074 \quad 0.3322$

HORMALIZEO TELLURIC TRAHSFER TEMSOR

$-0.2641-0.2386-0.0866-0.0544$

$\begin{array}{llll}1.2966 & 0.2792 & -0.2992 & -0.0991\end{array}$

$\begin{array}{llll}-0.3152 & 0.6720 & 1.1246 & -0.0569\end{array}$

$F-1$

FARMIHETOH (BASE)

FA-E-1 (\$4)Q:

PEFIOL: 1 BEE

Aิำ: 1.5

PHASE-CORFECTED TOMPEISEATED TELLURIC

ELLIFSE PARAMETERS

MÂJUR HYY.TS: 1.475

MINUR AXIS: E.855

AEIHUTH: $\quad-41.369$

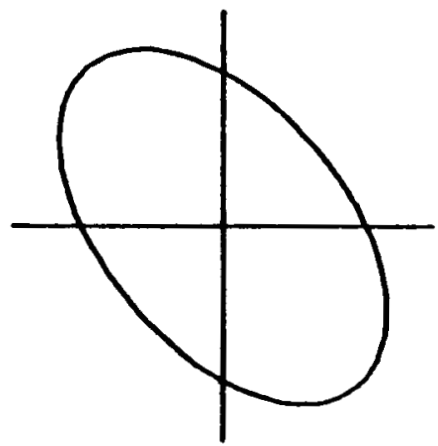


IHPUT IHPEDANCE TENSOR

$\begin{array}{llll}-0.0399 & 0.0263 & 0.2166\end{array}$

$-0.1943-0.2758 \quad 0.0232$

0.2505

0.0032

NORMAL IZED TELLURIC TRAMSFER TEMSOR

$1.04460 .0760 \quad 0.0301-0.1476$

$0.0591-0.0446 \quad 1.0512 \quad 0.1824$

$F-2$

FHFHIHGTOH (EASE)

FA-E-3 (IOA2:

PEPIOLO: 1099

MXIS: 1.5

PHAEE-CORRECTE[

COIAFEHSATEO TELLUPIC

ELLIFSE FARAAHETERS

IHGJOP AGIS: 1.093

MIHIJE ARIS: 1.653

HEIHUTH: 47.503

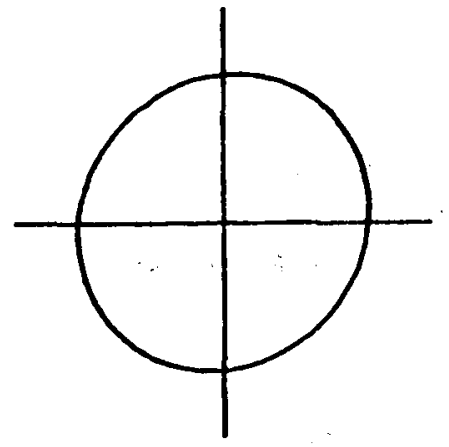

IHFUT IHFELAHCE TEHSOR:

$-0.0315-0.0992 \quad 0.2929 \quad 0.2692$

$-0.2103-0.2568-0.0031 \quad 0.0052$

COMFEHSATEC TELLURIC TRAHSFEF TEHSOR:

$1.03350 .1302 \quad 0.0911-0.0499$

$0.0147 \quad 0.0186 \quad 1.0444 \quad 0.104 i$

FAF!I:HETOLA REASE）

TS-E-Z

FEFIOE: 10 in

คำ15: 2

TOUTEEISATED TELLUR IC

ELLIFEE FÁRAHETERS

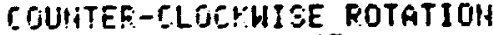

MP JUF fiols: $1.14 \mathrm{r}$

HIIlOF: A.PL: 1.923

GEIHITH: 35.937

CLOCYHISE POTATIJH

ViA JOF AGIS: 1.048

HIIHIF AYIS: 0.967

GZIMUTH: $\quad 62.656$

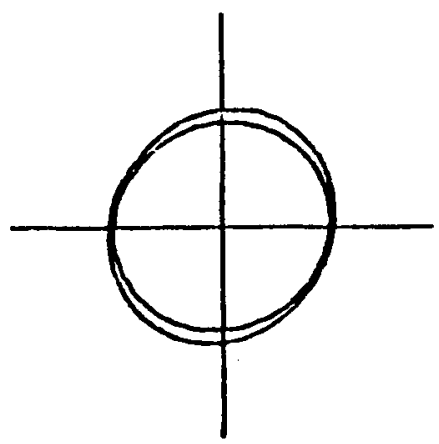




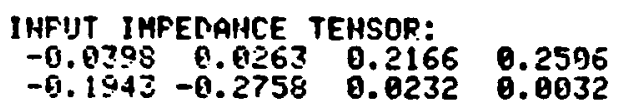

COMPENSATEL TELLURIC TRANSFEF TENSOR:

$\begin{array}{lll}1.0446 & 0.0769 & 0.0301-0.1476 \\ 0.0591-0.9446 & 1.0512 & 0.1824\end{array}$

FAFHINGTOH (BASE)

FA-E - Z ( $42 Z$ Z)

FEFIOT: 1900

ARIS: 2

COMFEHSATED TELLURIC

ELLIFSE PHFIAIETEFS

CHINTEF-OLOCYHISE POTTATIOH

Hin JUF AYIS: 1.199

NIIHUF 4\%15: 1.012

ÄIMITH: -S.EZ4

CLOILWISE PCTATIOH

Hí.JOF AOIS: 1.146

MINÜF A:21S: 0.867

ALINUTH: 71.832

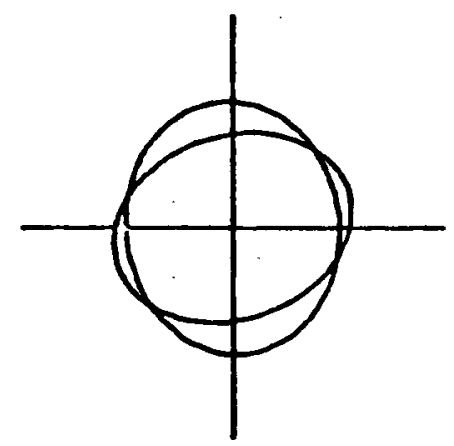

IHFUT IMPEDANCE TENSOR $-0.0615-0.0085$ O. 1691

$-0.0593-0.0896$

0.0104

0.1972

$0.016 ?$

MOFHALIZEO TELLURIC TRAHSFER TENSOR

$0.8190 \quad 0.05290 .1564-0.1184$

$0.0604 \quad 0.0141 \quad 0.3340 \quad 0.0665$

JE - BASE

IEHEZ MOENHTAIHS (BASE)

JE-E-1 (ST.45)

FEFIOD: 1609

15: 1.5

FHHSE-COFFECTEO CONFE!H BHTE! TE!LLUPIC ELLIFSE PAFGIUETEFS

HAJINR AY:IS:

MI!HOF AXIS: NZIHUTH:

0.844

9.313

9.654

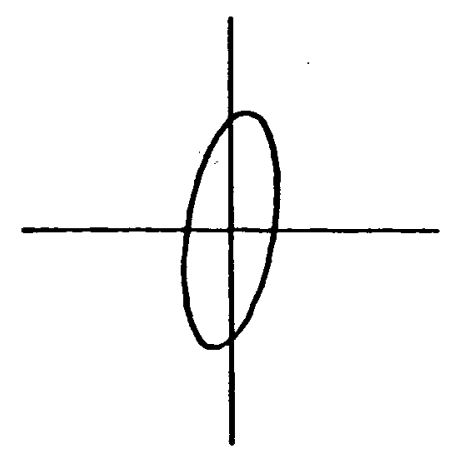


c)

IHFUT IHPEDAHCE TENSOR:

$\begin{array}{lllll}-0.0 E 15 & -0.0065 & 0.1691 & 0.1972\end{array}$

$-0.0598-0.0896 \quad 0.0104$ 0.01E

COMPENSATED TELLURIC TRANSFER TENSOR:

$0.8190 \quad 0.56290 .1564-0.1184$

G. DEG $0.0141 \quad 0.3340 \quad 0.0665$

IEVEZ MOUMTALIHE CEASE;

IE-E:-1 (A3AS)

FEE IOIS: 1595

H.:S:

COAREMGATED TELLUTEIC

ELLIF EE FHETATIETEF'S

COUHTEF-CLOCIRISE POTATION

NA JUT 4 OE: 9.950

M!IIJF A.:1S: 0.539

MEIIUTH: 4.95

CLOL!WIEE FGTATIÜH

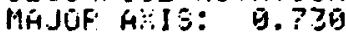

MINIJF Gals:

HEIHUTH: 15.972

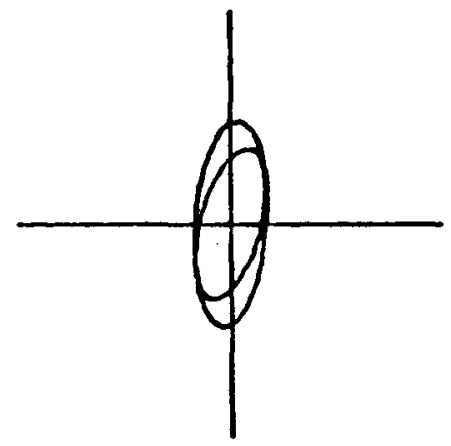

IHFUT IMFEDAHCE TEHSOR:

$-0.0796-0.071 ? \quad 0.2106 \quad 0.1489$

$-\overline{0} .0720 \quad-5.1048$ 0.0057 0.0039

COMFENGATEL. TELLUFIC TRAHSFER TENSOR:

$0.8938-0.13910 .3393-0.0176$

$0.9215-0.0940$ 0.3954 0.0733

JEMEZ MOLUHTAINS (BASE)

IE-E:-2 (n:4)

FEFIOTO: 1900

A.?IS: 2

COMFE!YSHTED TELLURIC

ELLIFSE FATEAHETERE

COU!ITEP-CLOCKLISE POTATIOH

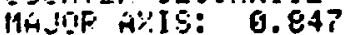

MIHîf A.:IE: 0.392

GEI!IUTH:

3. 643

CLOIVHIEE POTATION

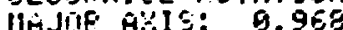

HIHUF GXIS: G.Z̈TE

MEIMUTH: 19.924

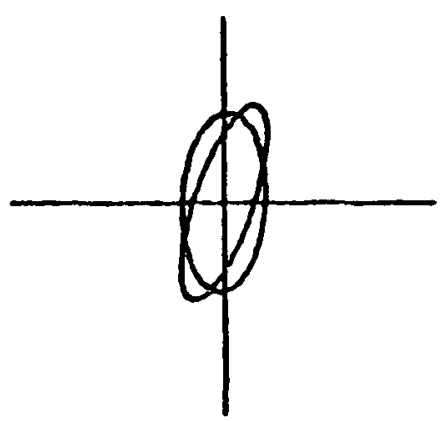




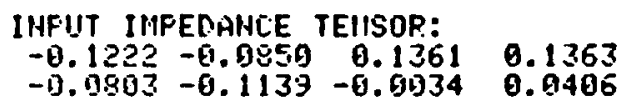

JEIIEZ HJUHTANIHS (BASE)

IE-ESZ-2 (A3TS)

FEF IOT: 10190

Mis: 2

EOHFEHSATED TELLURIC

ELLIFSE PHRGHETERS

CDUNTEF-CLOCKHISE ROTATIOH

iT JUP H IS: 5.902

HIHUF HiIs: 0.454

HEIINJTH: 25.449

LLOCKHISE ROTATIOH

MA JUE AHIE: O.PE?

MINIT AOIS: 0.132

ÄIMITH: ZE. 758

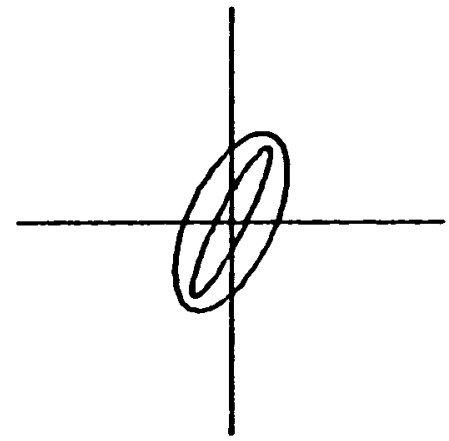

\footnotetext{
IHFUT IMPEDAHCE TEHSOP:

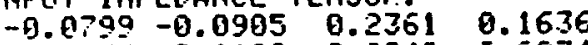

$-0.0936-0.1122-0.0042-0.0674$

COHFEHSATED TELLURIC TRANSFER TEHSOR:

$\begin{array}{llll}11.8536 & -11.1621 & 0.3809 & 0.0239\end{array}$

$-0.0259-0.19972$ B. $4378 \quad 0.6638$
}

IEMEZ-EACA (BASE)

IE-ES1-1 (A335:

PEC IOC: 10Й

$4 \div 15: 2$

TOMPENSATEO TE!LLURIC

ELLIFSE PARAAHETEFS

COIITTEP-CLOCKHISE POTATION

11.jOF AIS: 0.89 ?

IIHT MU:

HEIHISTH:

1. 521

CLDIKWISE ROTATIDH

HG JUF FOיIS: 1.191

HIHOR AOIS: 0.352

A2 IHISTH: $\quad 15.275$

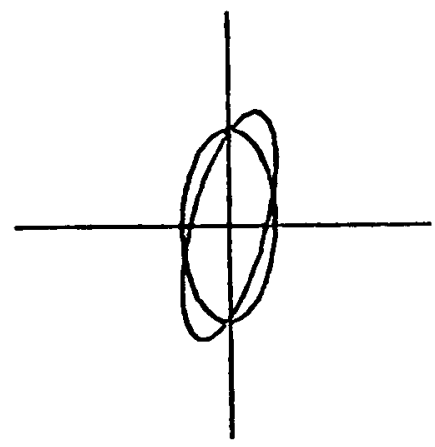


INPUT IMPEDANCE TENSOR

$\begin{array}{llll}-0.1851 & -0.1848 & 0.3048 & 0.2769\end{array}$

$-0.0618-0.0317-0.0297-0.0401$

NORMAL IZED TELLURIC TRANSFER TENSOR

$1.3005-0.0623 \quad 0.8271-0.0007$

$-0.1560-5.0233 \quad 0.2090-0.0672$

\author{
JE - 2 \\ JEAEZ (SATELLITE) \\ JE-ESZ-2 ( $380 \%$ \\ FERIOO: 1000 \\ AxIS: 1.5 \\ PHASE-CORRECTED \\ COMFEHSATED TELLURIC \\ ELLIFSE FAREAHETEFS \\ MH.JOR AXIS: 1.541 \\ MINUP A\%IS: 0.269 \\ AEINUTH: $\quad-6.744$
}

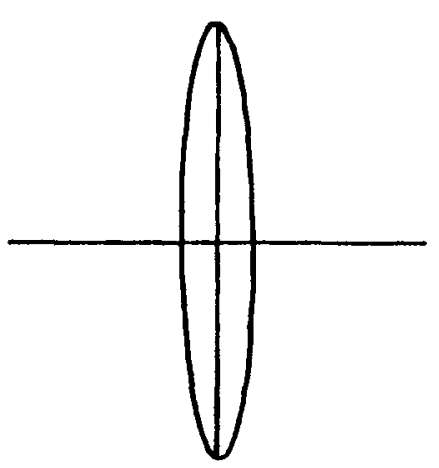

IHPUT IMPEDAHCE TEIISOR:

$\begin{array}{llll}-0.1851 & -0.1848 & 0.3048 & 0.2769\end{array}$

-0.06.18-0.8317-0.0297 -0.0401

COFPE!HSATED TELLURIC TRANSFER TENSOP:

1. $0005-0.5623$ 9.8271 -0.000 ?

$-\overline{0} .1560-0.9233 \quad 0.2090-0.06 .32$

IEMEZ (SATELLITE)

JE-EST-2 (\# 380 )

FEFIOT: 1000

ABIS: 2

COMPEHSATED TELLUFIC

ELLIFSE PAPANHETEPS

COUHTEF-CL GCYHISE POTATIOH

MH JOTF A.RIS: 1.599

HIHUF G.:15: 9.205

MEIMLTH: G. ER?

CLUEKHISE FOTATIOH

HAJJF: A. IS: $1.57 E$

HIHJP AिIS: 0.318

AEIIUTH: $\quad-2.807$

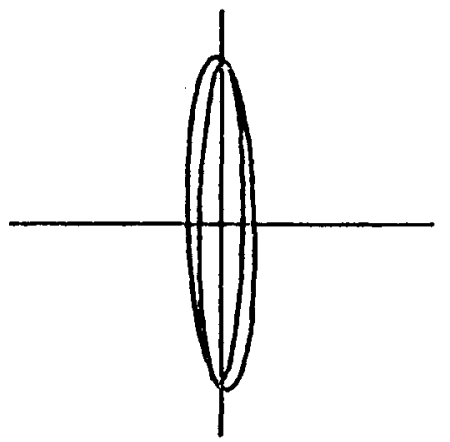


IHPUT IMPEDAHCE TEHSOR:

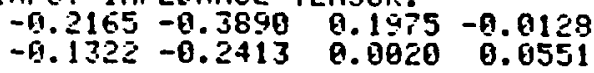

COIIFEHSATET TELLURIC TRAHSFER TEHSOR:

$\begin{array}{cccc}0.4131 & -0.4793 & 1.3539 & 9.3856 \\ 0.1275 & 0.1187 & 0.8352 & 0.2440\end{array}$

JEMEZ ISGTELLITE;

IE-EST-1 OAZAT:

FEFIOO: 1996

A.:1::

COMFEHSATEL TELLUFIC

ELLIFEE FARGMETERS

LOUHTET-OLOCKUISE RÜTATIOH

MA JOFF ÂIS: 1.302

HillOP A.:IS: 0.099

HIIHITH: $\quad 4 \hat{\imath} .419$

LLICKUISE POTATIOII

HAJUF AOIS: 2.148

HIHOP AOIS: 0.049

AEIHUTH: ZZ.032

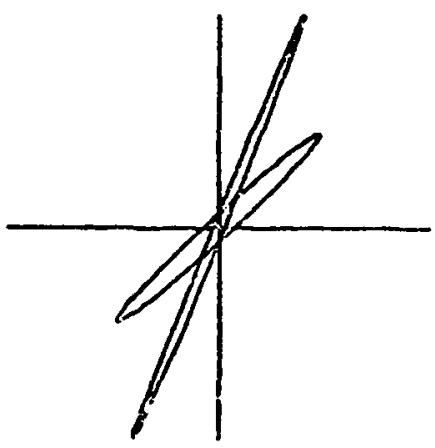

IHPUT IMPEDANCE TENSOR

$-0.2165-0.3890 \quad 0.1975-9.0128$

$-0.1322-0.2413$

$\begin{array}{rr}0.1975 & -9.0128 \\ 0.0020 & 0.0551\end{array}$
MORMALIZED TELLURIC TRAHSFER TEHSOR

$\begin{array}{lllll}0.4131 & -0.4703 & 1.3539 & 0.3856\end{array}$

$\begin{array}{llll}0.1276 & 0.1187 & 0.8352 & 0.2446\end{array}$

$J E-3$

JEMEZ (SATELLITE)

JE-ES3-1 (\$343)

PERTOOD: $190 B$

AXIS: 1.5

PHASE-CORPECTED

COMFENSATED TELLURIC

ELLIFSE PAFAHETERS

MAJJTE A:IS: 1.645

HIHOF AYIS: D.105

A? !HITTH:

35.797

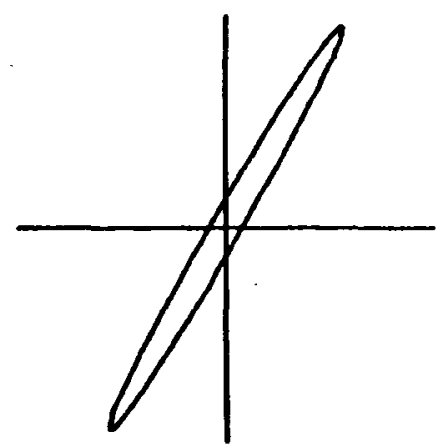


IHPUT IMFEDAHCE TEHSOR

$\begin{array}{lllll}-0.1014 & -0.1168 & 0.2276 & 0.1701\end{array}$

$-0.1398-0.2500-0.0919-0.0330$
MORMALIZEO TELLURIC TRAHSFER TEHSOR

$0.8893-0.1287 \quad 0.48790 .0346$

$\begin{array}{llll}-0.2793 & 0.1315 & 0.8715 & 0.2463\end{array}$

\author{
$\mathrm{JE}-5$ \\ JEMEZ (SATELLITE) \\ JE-555-2 (354) \\ PERIOD: 1000 \\ H.SE: 1.5 \\ FHASE-GORRECTED \\ COMFEHSATED TELLUFIC \\ ELLIFSE PARAMTETERS \\ $\begin{array}{ll}\text { MAJOF AXIS: } & 1.055 \\ \text { HINOF HXIS: } & 0.856 \\ \text { AZIMUTH: } & 35.796\end{array}$
}

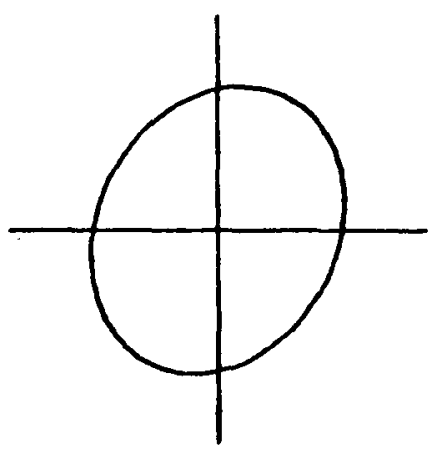

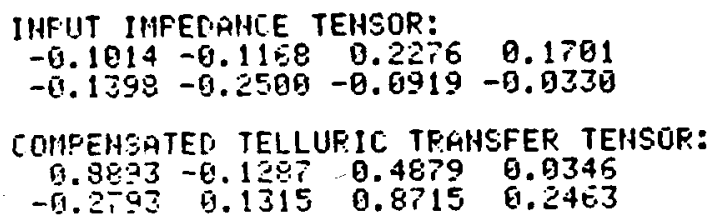
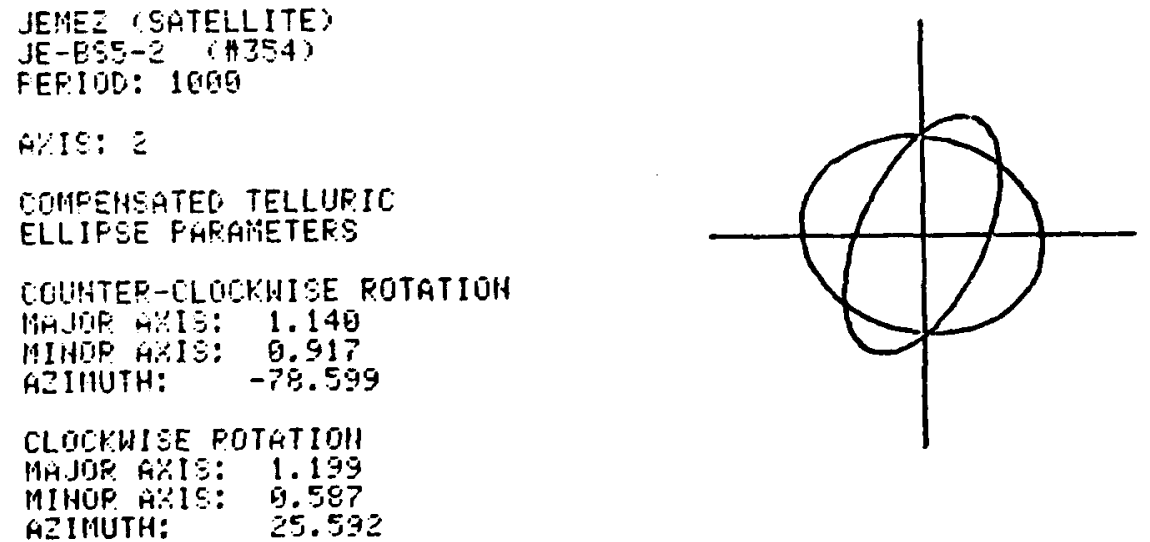
INPUT IMPEDANCE TEHSOR

$\begin{array}{lll}-0.0504 & -0.0661 & 0.1503 \\ -0.0619 & -0.0934 & 0.0279\end{array}$

0.0038

PRET-E\%:

0.918

PRED-EY:
NORMALIZED TELLURIC TRANSFER TENSOR

$\begin{array}{llllll}0.6461 & -0.0263 & 0.2604 & 0.0351\end{array}$

$\begin{array}{llll}0.0691 & -0.0556 & 0.3474 & 0.0704\end{array}$

JE -10

JEMEZ (SATELLITE)

JE-BS10-1 (" 368 )

FERIOD: 1000

A\%IS: 1.5

PHASE-CORRECTED

COMPEHSATED TELLURIC

ELLIPSE PARAMETERS

HAJOR AKIS: 0.728

MINOR AKIS: 0.284

AिZIMUTH: $\quad 18.456$

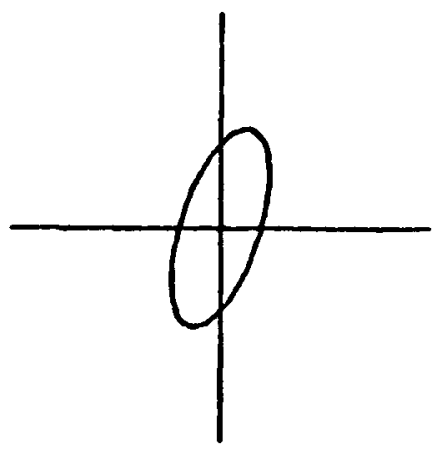

INPUT IHFEOAHCE TEHSOR

$-0.0594-0.0651 \quad 0.1503$

$-0.6 \leq 19-0.0934 \quad 0.0279$

0.1386

0.0030

PREC-EY: 0.918 PRED-EY: 0.876
HORMALIZED TELLURIC TRANSFER TENSOR

$0.6461-0.0263 \quad 0.2664 \quad 0.0351$

$\begin{array}{llll}0.0691 & -0.0556 & 0.3474 & 0.0704\end{array}$

$J E-10$

JEMEZ (SATELLITE)

JE-B.S10̈-1 ( $3 E 8)$

PERIOD: $100 \overline{0}$

Aै:IS: 2

COHPEHSGTED TELLURIC

ELLIPSE FARAHETERS

COUHTER-LLOCKNISE ROTATION

MA JÜF ÂXIS: $0.6 E 4$

MIHOF AXIS: 0.269

GEIMUTH: 19.744

CLOCKNISE ROTATION

HGJOP AXIS: 0.802

HIHOF AXIS: 0.293

AZIMUTH: 24.572

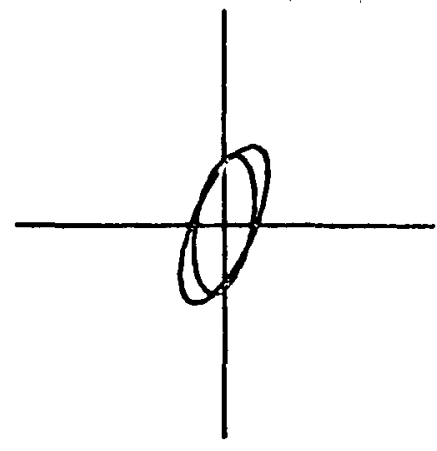


INPUT IMPEDANCE TEHSOR

$\begin{array}{llll}-0.0332 & 0.0233 & 0.2045 & 0.0944\end{array}$

$-8.0641-0.0992-0.1364-0.0796$

$J E \cdot-11$

JEHEZ (SATELLITE)

JE-ES11-1 (B370?

FEFIOC: $18 B D$

AXIS: 1.5

FHASE-COFFESTEO

COATFE!SEATED TELLURIC

ELLIF SE PAFGMIETERS

HA JOR ARIS: 0.847

HIHOT A.2IS: 9.308

A2IMUTH: -41.188
HORMALLIZED TELLURIC TRANSFER TEMSOR

$0.6683-0.2462 \quad 0.0356-0.1399$

$\begin{array}{llll}-0.4816 & 0.1282 & 0.3651 & 0.0784\end{array}$

\footnotetext{
IHPUT IHPEDAHCE TEHSOR:

$\begin{array}{llll}-0.0392 & 0.0233 & 0.2045 & 0.0944\end{array}$

- $6.0641-0.0992-0.1364-0.0790$

EDMFEMSATED TELLURIC TRAHSFER TENSOR:

B. $6683-0.2462 \quad 0.0356-0.1390$

$\begin{array}{llll}-19.4816 & 0.1282 & 0.3651 & 0.0784\end{array}$
}

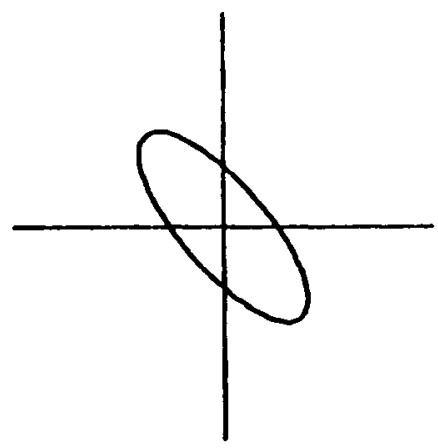

IEMEZ (SATELLITE)

IE-ES11-1

PEFICIO: 1000

Hi:IS: 2

COMPEHEATED TELLURIO

ELLIFSE FARAHETERS

COUHTEP-CLDCKHISE ROTATIOH

MA JOF: AिL15: 1.990

liiliúf 9.xis: 0.258

HEIMUTH: $\quad-41,454$

CLOCKMISE POTATIOH

HAJOR A.YIS: O. E?

MINOP AKIS: $9.35 E$

HEIHUTH: -3Z.210

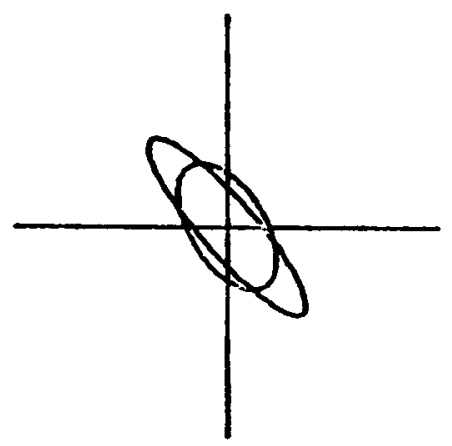


IHFUT IHPEOAHCE TEHSOR:

$\begin{array}{llll}-0.0368 & 0.0123 & 0.1845 & 0.1142\end{array}$

$-8.01 .13-0.1002-0.1362-0.0762$

COHFENSATED TELLURIC TRAHSFER TEHSOF:

1. E. $79-15.1571 \quad 0.0549-0.1098$

$\begin{array}{llll}-1.449 & 0.1343 & 0.3612 & 0.0379\end{array}$

IEVEE (SATELLITE)

IE-ECI1-1 (43?G)

FEFIOT: 1900

A:i: : 2

COIAFEHSATE!; TELLUFIC

ELIIPSE FARAIIETEPS

COUHTEFI-CLOEKHIEE PÖTATTIOH

I1M.OOF HFIS: 1.0338

MILUT H.IS: 9.316

HE!HITH: $\quad-43.414$

CLOCHHEE POTHTIOH

MH.JIF: G\%IS: G. ESE

MINIJP GLIS: 0.397

AZIHUTH: $\quad-32.682$

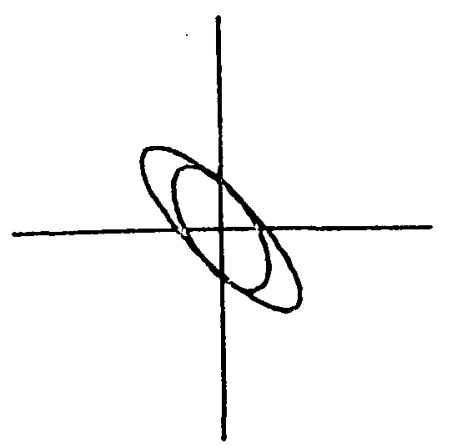

IHPUT IMFEDAHCE TEHSOR $-5.1431-0.0942 \quad 0.3233$

$-0.0971-0.17540 .0093$

0.2619

0.0478
HORMALIZED TELLURIC TRAHSFER TENSOR

$\begin{array}{llll}1.3084 & -0.1372 & 0.5305 & -0.1093\end{array}$

$\begin{array}{llll}0.1277 & 0.0860 & 0.6094 & 0.1750\end{array}$

B - I

JEMEZ-EACA (SATELLITE)

JE-ES1-1 (\#386)

FEFIOD: 10015

Axis: 1.5

FHASE-COFPECTEO

COHAENEATER TELLURIC

ELLIFSE FÁFÂHETERS

MAJUER A?IS: 1.469

MIHUF ÂIS: 0.500

A2IHITH: $\quad 15.710$

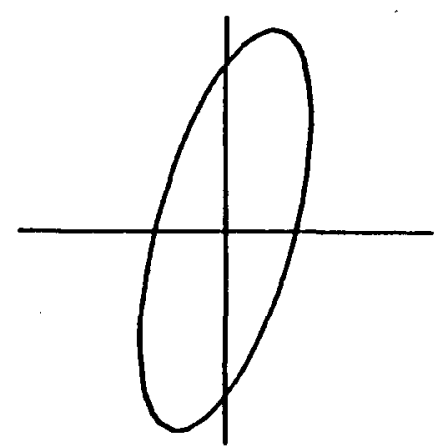




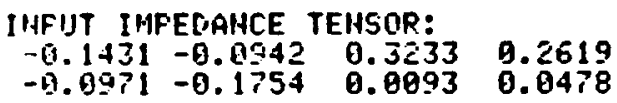

CIIPEISSATED TELLURIC TRAHSFER TEHSOR:

$1.3194-0.1372 \quad 0.5305-0.1093$

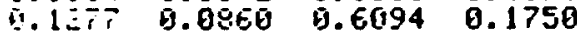
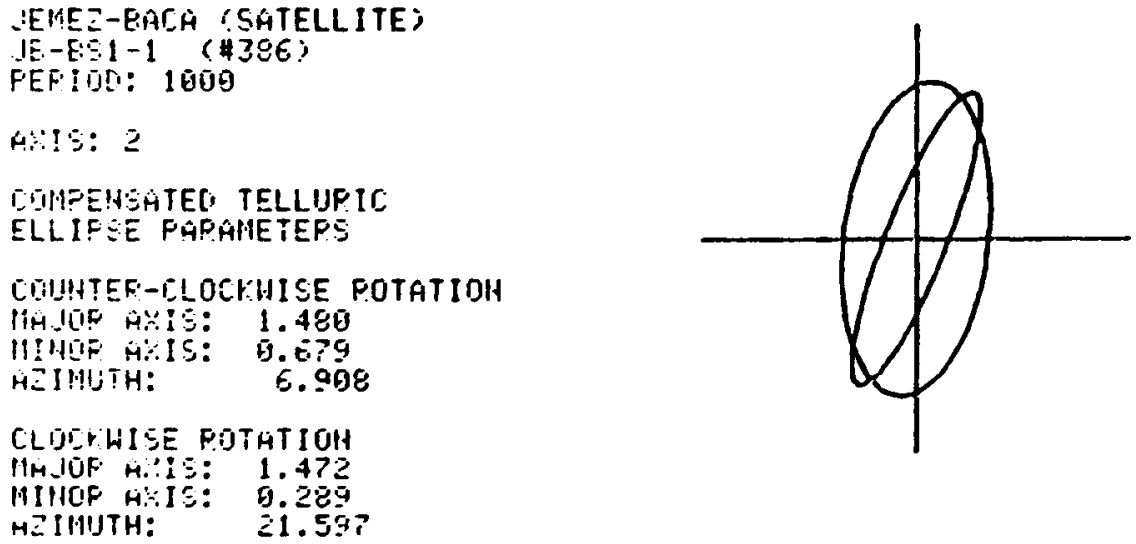

INPUT IMPEGANCE TEHSOR

$B-3$

JENEZ-EHACA (SATELLITE)

JE-ESZ-1 (HA1B)

FEFIOO: 1000

Arits: 1.5

PHASE-CDPEECTEL,

COHFEHSHTED TELLURIC

ELLIF'SE PARAMILTEPS

MH.JCF H.LIS: 1.149

MIHUF H.IS: 0.276

HEIMUTH: -5.845

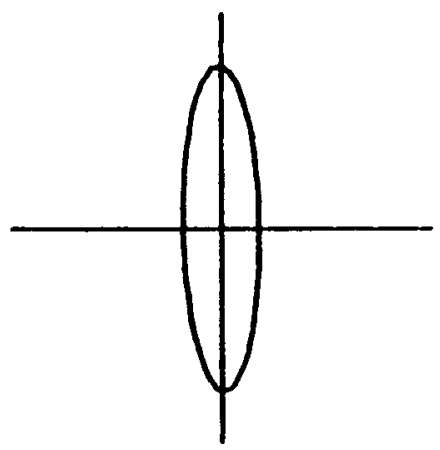


IHFIT IHPEDAHACE TELSOR:

G. $16210.07410 .23150 .246 \%$

-0.1581-0.0602 0.01840 .0176

COMFENSATEO TELLURIC TRANGFEF TENSOR:

1. BE.96 $0.0341-0.3949 \quad 0.0624$

$0.9505-6.0919 \quad 0.2614 \quad 0.0047$

!E MEZ-EACÁ (SATELLITE)

$J E-E Z Z-1$ (\$413)

FEFIOC: 1 GQñS

His: 2

TOMFËHSATE[ TELLUF IC

ELLIFSE PGFAMETEFS

COIMHTEF-ELOEKHISE FITTATIOHA

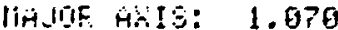

HIIUF: A.XIS: $9.2 ? 3$

HEIMIJH: -9.979

CLOLFHISE POTATIOH

MA JIF AOEIS: 1.210

MIHIJF AXIS: G.

HEIHUTH: -

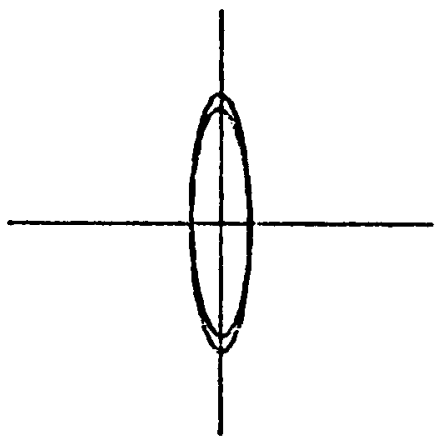

IHPUT IMFEDAHCE TENSOR

$0.0696 \quad 0.0467 \quad 0.2339$

6. 1790

HORMAL IZED TELLUPIC TRAHSFER TENSOR

$-0.0697-0.1255 \quad 0.1086$

0.0434

$0.9031-0.1436-0.1259-0.0831$

$0.3398-0.1457 \quad 0.4365 \quad 0.1246$

B- 4

JEHEZ-BATEA (SATELLITE)

IE-ES4-1 (\#417)

FERIOQ: 1009

AXIS: 1.5

PHASE-CORPECTEO

COIMPEHSATEO TELLITRIC

ELLIFSE FAFAMETEPS

MH.JIF AXIS:

MIHOR AKIS:

0. 965

HEIHUTH:

0.452

21.896

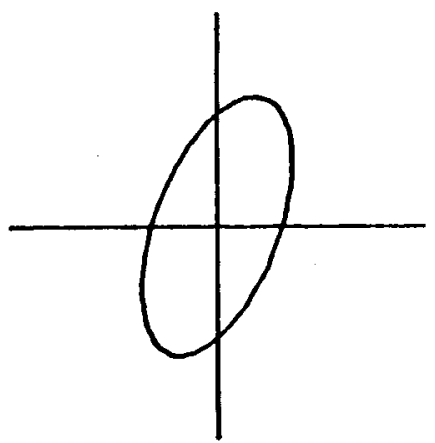




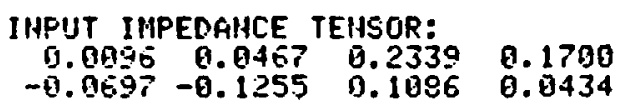

COMPEHSATED TELLURIC TRAHSFER TEHSOR:

9. $9031-0.1430-0.1259-0.0831$

i. $3395-9.145 \% \quad 0.4365 \quad 0.1246$

IEMEZ-EACA CSATELLITE)

IE-E $34-1$ :41??

FEF IOL: 1909

HOBO: 2

CATFESHATED TELLUP IC

ELLIF'E FARAAHETERS

COUHATEF-ELGCKHISE RTJATIOH

lif !OT f.:S: 1.0.32

MIHOF AKIs: 0.334

AEI!UTH: 8.171

CLIJTHISE PDTAT!ÜH

MH.JOF $4: 15: 1.904$

HIIIOF A.RIS: 5.46 ?

HEIHUTH: $4 \dot{0} .704$

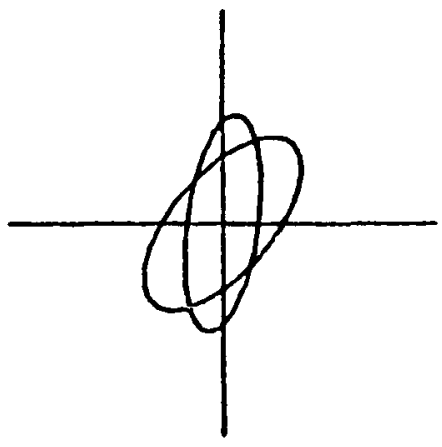

INFUT IMPEDAHCE TENSOR

$\begin{array}{llll}0.5591 & 1.0882 & 0.5712 & 0.3043\end{array}$

NORMALIZEO TELLURIC TRAHSFER TENSOR

$-0.4575-1.5981 \quad 0.4965-0.2790$

$1.9575-0.5968-3.6834-1.1832$

$0.4730-1.7207 \quad 4.5963 \quad 2.5505$

LV - B

LAS VEGAS (RASE)

LU-E-2 O 309 ?

FEFIOD: 1000

A.YIS: 3

FHASE-COFRECTEO COMFENSATED TELLURIC ELLIFSE FAFGMETERS

MAJUR AYIS: $\mathbf{5 . 9 5 8}$

MIHOR AHIS: 1.892

AZIMUTH: -48.518

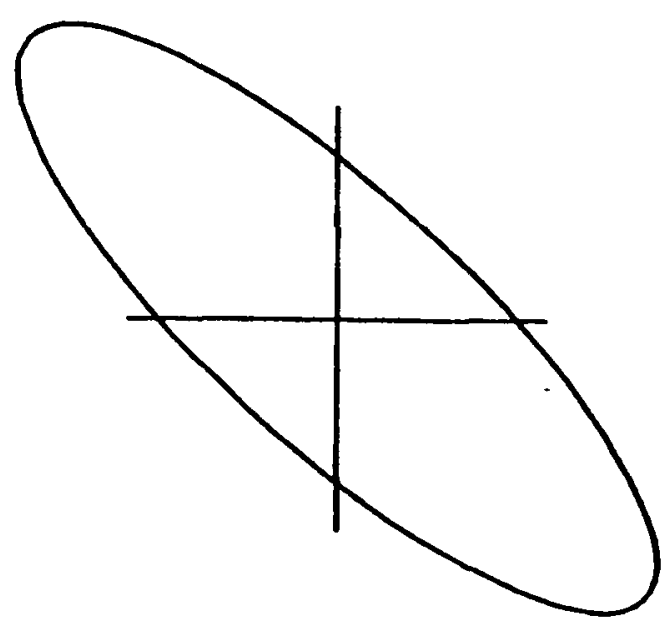




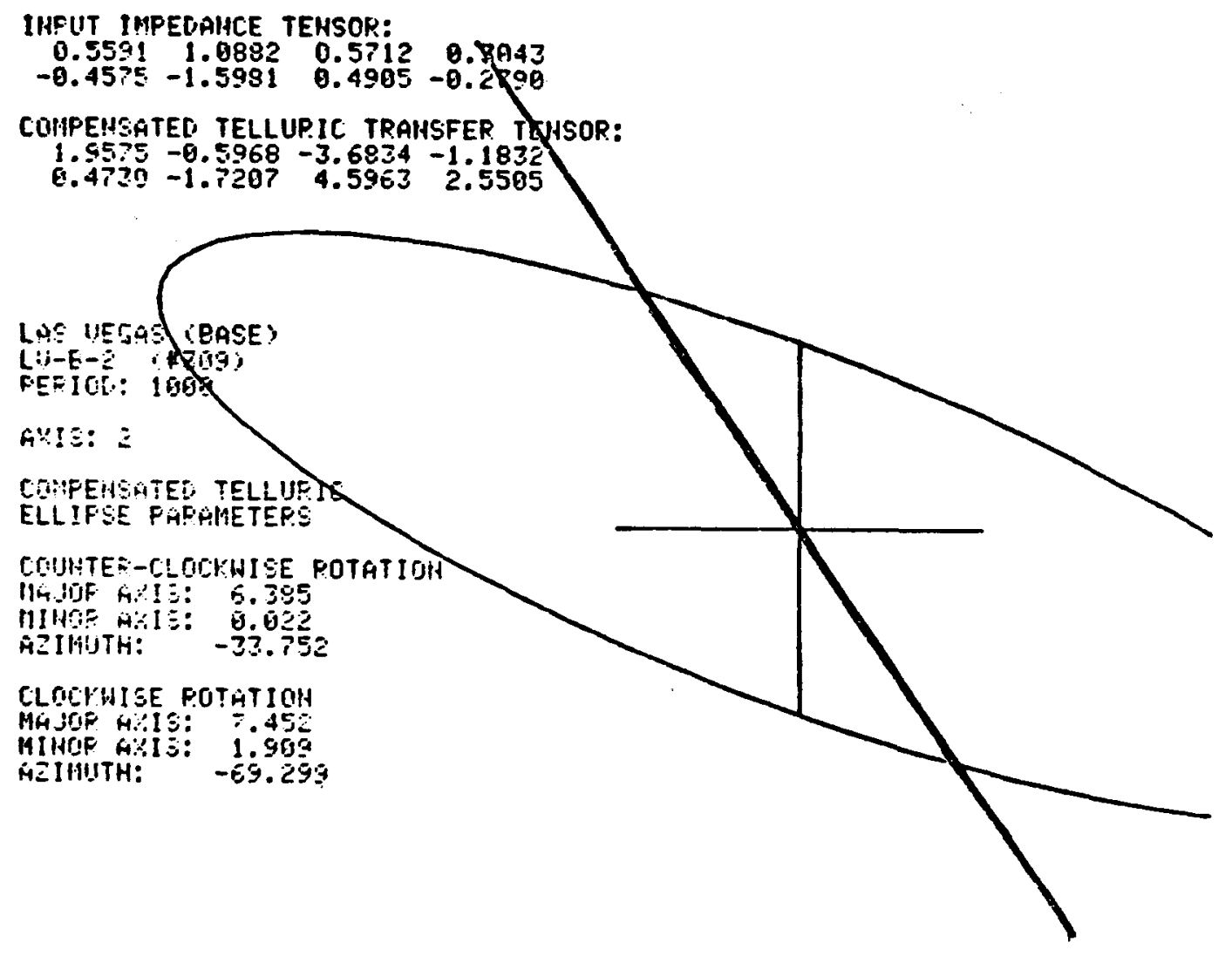

IHPUT IMPEQANCE TEHSOR

$\begin{array}{llll}0.555 \% & 0.4635 & 0.9990 & 0.6995\end{array}$

MORMALIZED TELLURIC TRAHSFER TENSOR

$-0.8420-0.9479-0.3311-0.0757$

$3.7978-6.6697-2.2791$
-0.9996

0.2061

(1)

$-8.075$
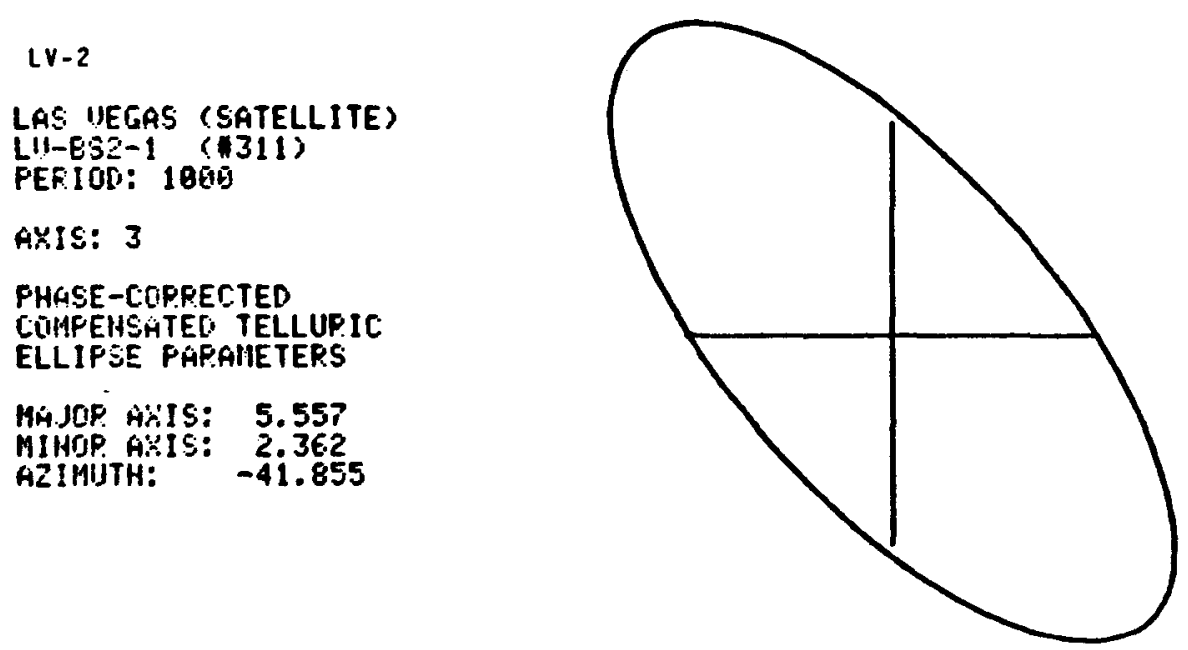
IHFUT IIPPEDAHCE TEHSOR:

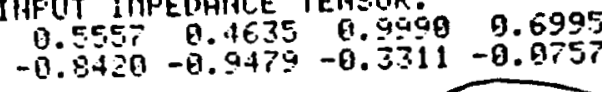

$-0.8420-0.9479-0.3311-0.075$

CONFEHSHTED TELLURIC TP AHSFER TENSCD.

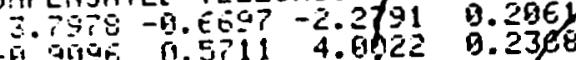
- Ex. ATing
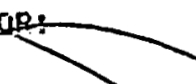

LGS IETOES (SATELLITE)

L:LES:- (H311)

FEFIOLI: 19000

ก:I5: 2

COMFEHEATEL TELLURIC ELLIPSE PGEAHETEFE

COUHTEF-LLQLILISE POTATIOH

MÂJOE AXIS: $6.28 E$

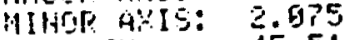

AEIMUTH: $-45.51 E$

CLOEKHISE FITATIOHA

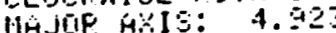

MIHITE HAIS: 2.579

GIINUTH: -34.36E

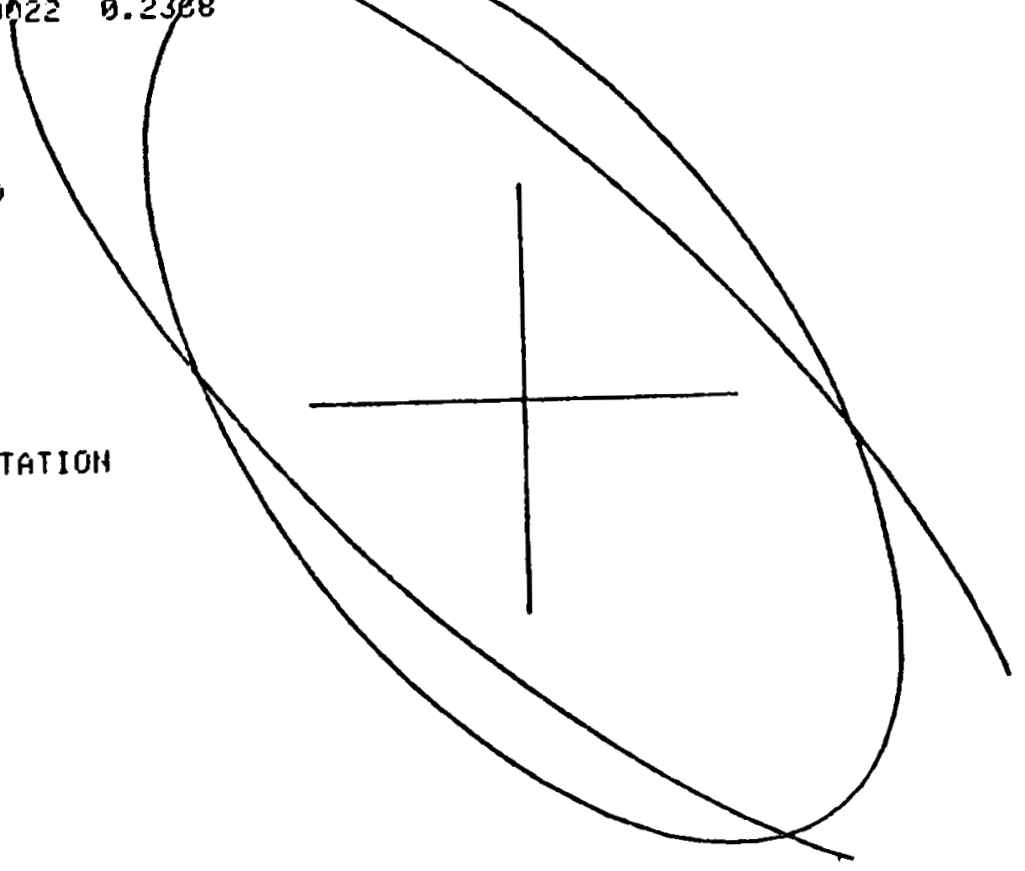

\section{IHFUT IHFEDANCE TEHSOR \\ Q. 008 ? 0.02390 .21110 .1470 \\ MORMAL I ZED TELLURIC TRANSFER TEHSOR \\ $\begin{array}{lllll}-9.0801 & -0.1094 & 0.0049 & -0.0394\end{array}$ \\ $0.8067-0.1434-0.0728-0.8339$ \\ $\begin{array}{llll}-0.0772 & -0.0989 & 0.4238 & 0.0657\end{array}$}

SF - 3ASE

SANTE FE (EASE)

SF-E:S $3-4$ ( $\$ 350)$

FERIO0: 1905

AVIS: 1.5

FHÂE-CORPECTER COMPEHSATED TELLURIC

ELLIFEE F'HFAMETERS

HAH JOF AXIS: 9.815

MINUF GOLS: 0.459

GEINUTH: -19.940

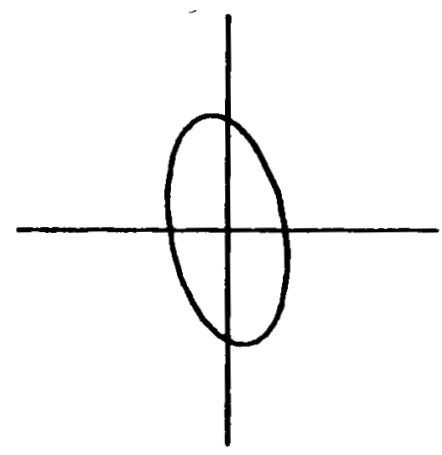


INPUT IMPEDANCE TENSOR:

$\begin{array}{llll}-0.0065 & 0.1232 & 0.1954 & 0.1237\end{array}$

$-0.1146-0.1237-0.0110-0.0472$

COMPENSATED TELLIIRIC TFAHSFEP TEHSCIF:

$0.6911-0.1290$ - E. GIZT - S. EEE

$-0.1303-1.6809 \quad 0.5330 \quad 0.0203$

SANTE FE (BASE)

SF-B-2 $(\$ 321)$
PERIOO: 1000

Ax15: 2

COMOENSATED TELLURIC

ELLIPSE PARAMETERS

COUNTER-CLOCRWISE RUTHTIOH

MAJOR AX!S: 2. 82:

MINOR AXIS: $0.3 E 5$

AZIMUTH: $\quad-2 Z . E E S$

CLOCKNISE ROTATION

MAJUOP AXIS: 0.638

MINOR AXIS: O.EI?

AZIMUTH: -34.212

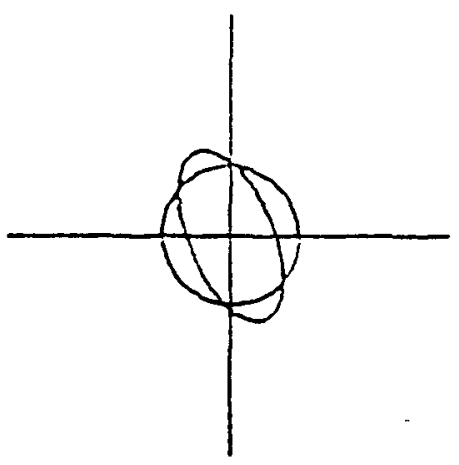

INAUT IMPEDANCE TEHSOR:

B. GOS 0.0339 0.2111 0.1470

$-0.0801-9.1094 \quad 0.0049-0.0394$

COHFEHSATEO TELLURIC TRAHSFER TEHSOR:

$0.8007-0.1434-0.0728-0.0339$

$-3.0172-0.9939 \quad 0.42380 .065 \%$

CAHTE FE (BQSE)

SF-ESE-4 (HES?

FEFIOIS: 1909

$415: 2$

COTPEISGATES TELLURIO

ELLIFSE PÄFHHETEFS

ETURTEF-ELUCRHISE PUTTATIOH

MA UIF ACIS: 9.893

HIHIF $4 \therefore$ IS: 0.259

HEIHUTH: - \$5.

CLOCHMISE FOTATTIUAH

MA.JOF MXIS: B. TRZ

MIHITP AXIS: 0.521

ARIIIITH: $\quad 4.39$ ?

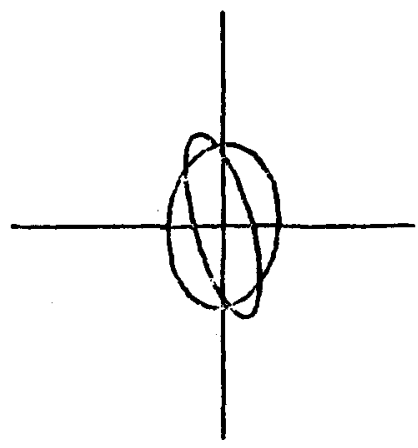


HAJOF AXIS: D.

HIHIOE AKIS: 9.469

AZ!MUTH: -13.251
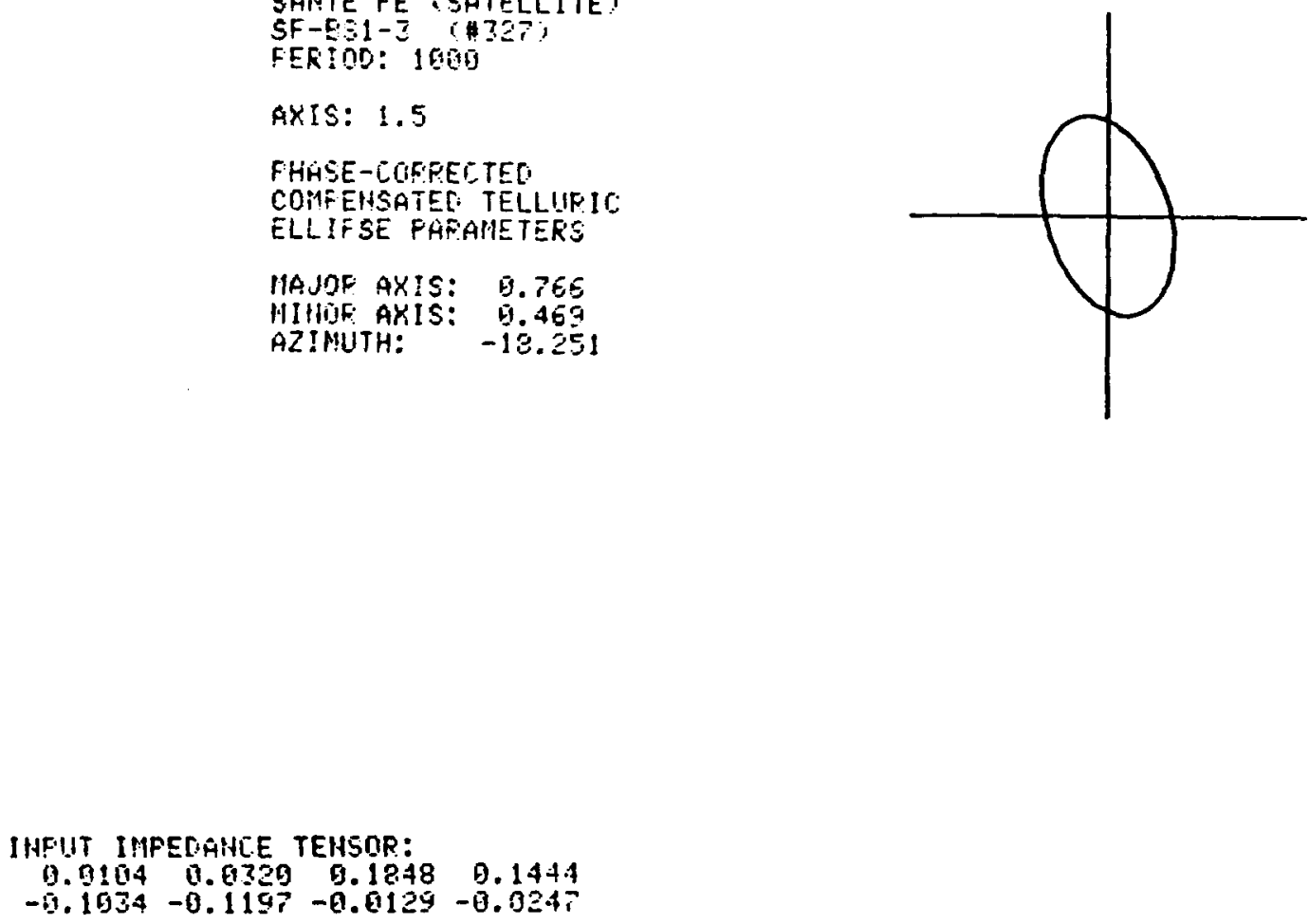


\section{IHFUT IMPEGAHCE TENSOR

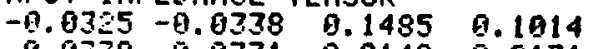 \\ - $.0758-0.0731-0.0149-0.0171$}

$S F-2$

SAISTE FE SATELLITE:

SF-ESE-2 C\#332?

FEFI IOC: 1050

AXIS: 1.5

FHASE- OOFFECTEO

COHFEHSHTES TELLUPIC

ELLIFSE FHFÂHIETEPS

MAJUT AOIS:

MIHOF AOIS:

AIIIIUTH:

6. 579

0.344

3.655
NORMAL IZED TELLUR IC TRANSFER. TENSOR

$0.5587-0.1053 \quad 0.14810 .0928$

$-0.0715-0.0051 \quad 0.3374-0.0104$
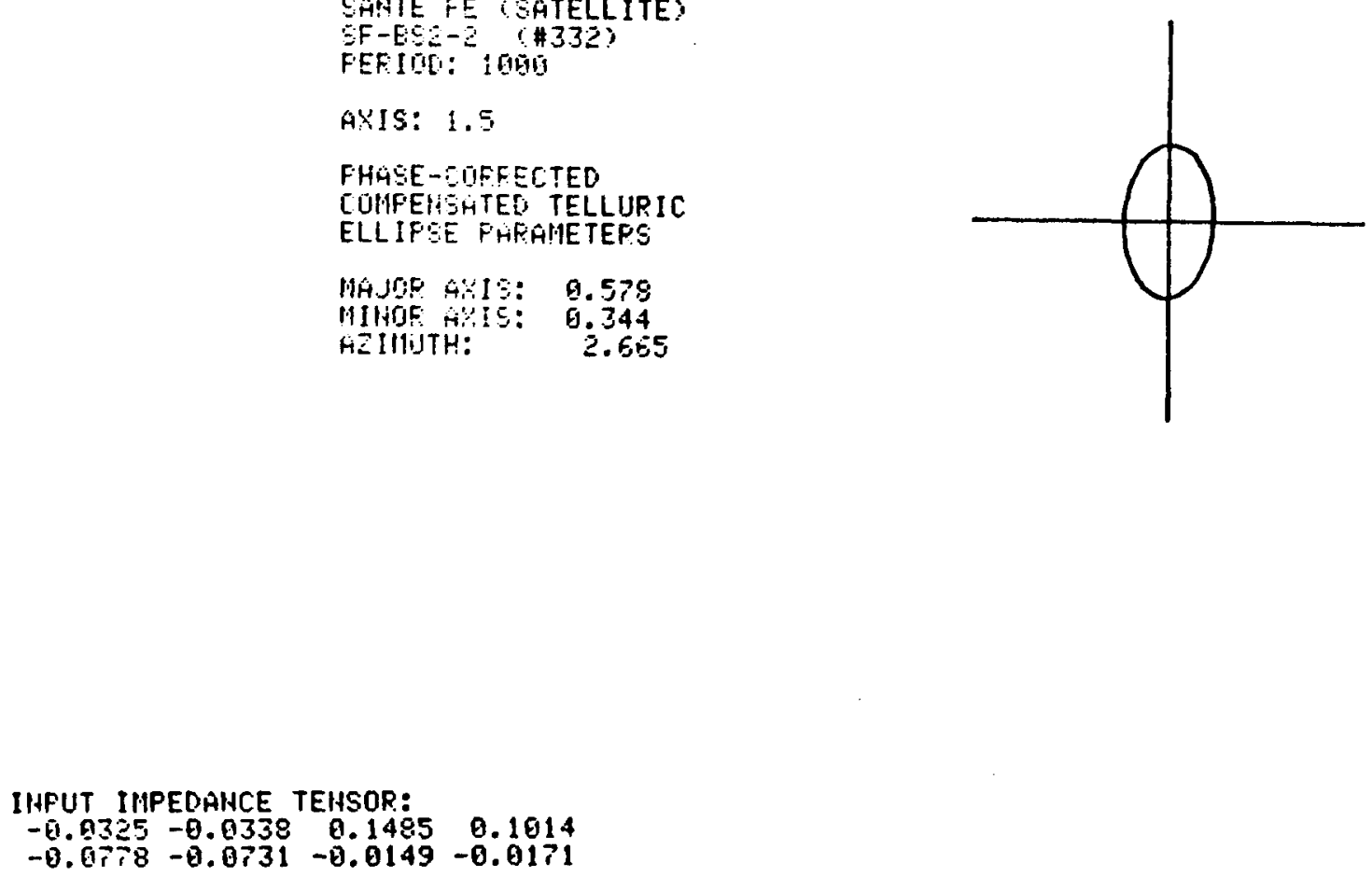
Normalized Telluric Ellipses (Unit Amplitude Circularly

Polarized electric Field)

$$
T=20 \text { Seconds }
$$

IHPUT IMPEDANCE TENSOR

$\begin{array}{rrr}-0.7814 & 0.1217 & 0.7029 \\ -6.3824 & -3.4264 & -0.7648\end{array}$

BOSE IMPEDAHCE TEMSOR:

BASE IMFEDAHCE TENSOR:

FRED-ES: 0.996 PRED-EY:

0.6352

0.9577

REMOTE TELLURIC TENSOR:

HORMALIZED TELLURIC TRANSFER TEHSOR

$0.4232-0.02140 .2086-0.2856$

$\begin{array}{lllll}0.0610 & 0.5447 & 1.2044 & 0.9626\end{array}$

PRED-EX: 6.623 PRED-EY: 0.722

JEMEZ-BACA LOCATION (REMOTE)

JE $-\mathrm{B} 5-\hat{\mathrm{A}}$

FEPIOR: 20

A.:15: 1

COMPEHSATEO TELLURIC ELLIFSE PARAMETERS

COUHTER-CLOCKHISE ROTATION

MHJOP AYIS: 1.975

HINOR GXIS: 0.P13

AZIMUTH: -84.708

CLOCKHISE POTATIOH

MAJTSP AOIS: 1.241

MIHOP AY.1S: 0.117

AZIMUTH: 8.739

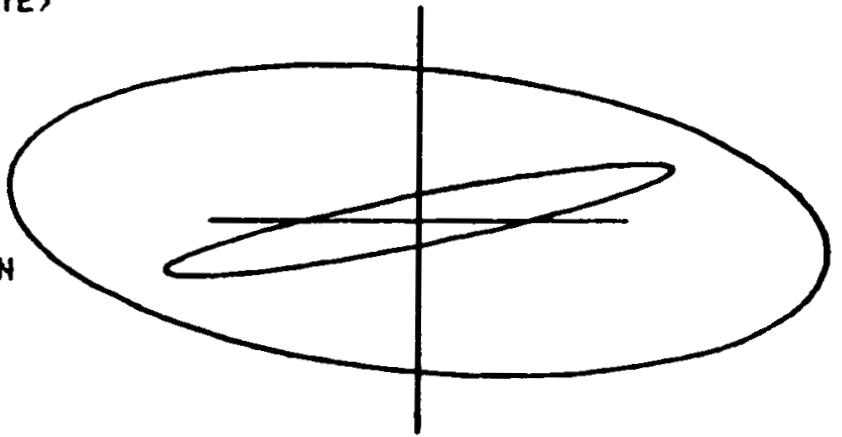

IHPUT IMPEDAHCE TENSOR

$-0.8395 \quad 1.1512 \quad 0.5336$

$-1.5019-4.1574-1.3935$

3. 3164

NORMALIZED TELLURIC TRANSFER TENSOR

EASE IMPEOAHCE TEHSOR:

B. 1726

$0.2688-0.0687-0.1082-0.6389$

F.FEL-E: 0.980

PRED-EY:

0.944

REMOTE TELLURIC TEHSOR:

PRED-EX: 0.757 PRED-EY: 0.83

JEMEZ-BACA LOCATION (REMOTE)

$\mathrm{JB}-\mathrm{EP}-\mathrm{A}$

PEFIOO: 20

AิำIS: 1

COMFENSATED TELLURIC

ELLIFEE F'ARAAIETERS

COUHTER-CLOOKKHISE ROTATION

MÂJOF: AXIS: 2.659

MIHDF HXIS: 0.763

AEIMUTH: -70.519

CLOCKHISE ROTATIOH

MA.JOF AिZIS: 1.393

MINOR GYIS: 9.337

A2IMUTH: -83.261

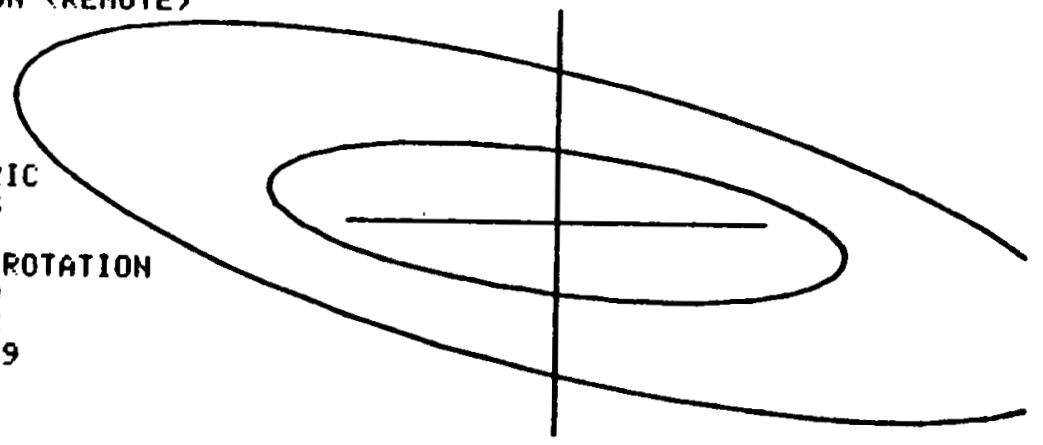


IHPUT IMPEDAHCE TEHSOR

$\begin{array}{rrr}-1.1419 & 0.3316 & 0.835 ! \\ -1.2578 & -2.8143 & -0.1494\end{array}$

1.0871

0.1886

REMOTE TELLURIC TENSOR:

BASE IMPEDAHCE TENSOR:

FFEQ-EX: 0.906

PREO-EY:

0.944
MORMALIZED TELLURIC TRAHSFER TEHSOR

$0.6078 \quad 0.0797 \quad 0.2563-0.4660$

$0.0124 \quad 0.1069 \quad 1.28780 .4922$

PRED-EK: 0.94 PRED-EY: 0.989

JEMEZ-BACA LOCATION (REMOTE)

JE-EE-Á

PERIOQ: 28

AำIS: 1

COMPENSATEO TELLURIC

ELLIFSE PARAMETERS

COUHTEP-CLOCKNISE ROTATIOH

MAJOP AYIS: 1.476

HIHOR AYIS: 1.124

GEIHUTH: -87.065

CLOCKUISE ROTATION

MGJOR AXIS: 1.303

MINOR AYIS: 0.060

AZIMUTH: 89.338

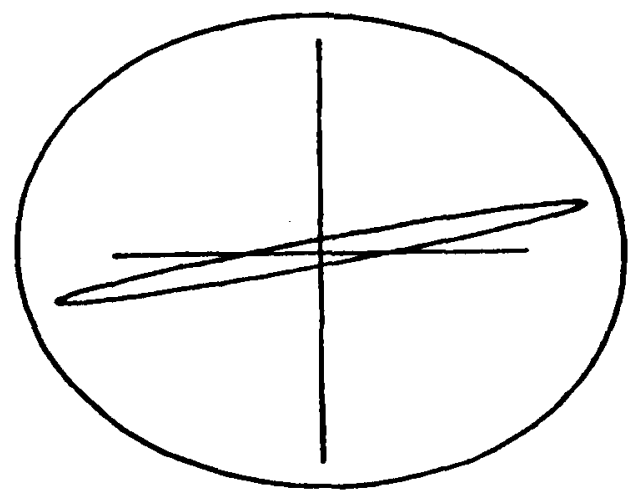

IHPUT IMPEDANCE TEHSOR

$\begin{array}{llll}-1.0942 & 0.1018 & 0.4511 & 0.6022\end{array}$

$-1.1359-2.2656-0.2372-0.0536$

BASE IMPEDANCE TEHSOR:

FFED-EY: $0.9 B 6$

FRED-EY: 0.944
NORNALIZEO TELLURIC TRAHSFER TEHSOR

$\begin{array}{rrrr}0.3331 & 0.0478 & 0.3138 & -0.3782 \\ -0.0920 & 0.0581 & 1.0756 & 0.3572\end{array}$

RENOTE TELLURIC TENSOR:

PRED-EX: 0.829 PREQ-EY: 0.956

JEMEZ-BACA LOCATION (REMOTE) JE-E:S-A

PEPIOT: 20

A\%IS: 1

COMPEHSATED TELLURIC

ELLIPSE PARAMETERS

COUHTER-CLOCK.WISE ROTATIOH

MAJUR AKIS: 1.223

HIHOR ÂIS: 0.792

ADIMUTH: 83.997

CLOCKWISE ROTATIOH

MAJOR AYIS: 1.080

HIHOR AYIS: G.158

AZIMUTH: 7G.

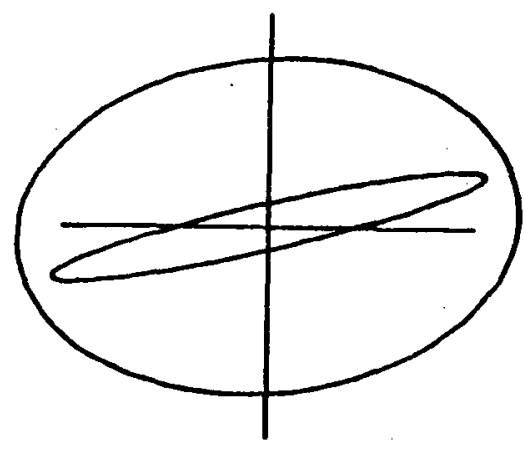


IHPUT IMPEDANCE TEHSOR $\begin{array}{llll}-3.1935 & 0.4992 & 2.6951\end{array}$

$-0.966 .6-2.5465-0.3867$

4.0566

0.3539

EASE IMPEDAHCE TENSOR:

FRED-EY: $0.90 E$ PRED-EY:

0.944

JEMEZ-EÁCA LOCATIOH (REMOTE)

JE-B $1 \mathrm{Q}-\hat{\mathrm{H}}$

FERIÚD: $2 \theta$

AOIS: 1

COMPEHSATED TELLUPIC

ELLIFSE FARGMETERS

COUHTER-CLOCKWISE ROTATIDH

MAJUR AXIS: 3.527

MIHOR A.OIS: 1.438

HZIMUTH:

0.513

CLOCKWISE ROTATIOH

MÂJOR AYIS: 1.351

HIHOR AXIS: 0.467

AZIMUTH: $\quad 44.508$
MORMALIZED TELLURIC TRANSFER TENSOR

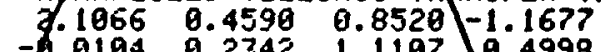

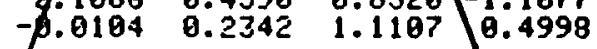

REMOTE TELLURIC TENSOR:

PRED-EX: U.7E6 PREO-EY: 0.706
IHPUT IMPEDANCE TENSOR

$-0.3414-0.320 \% \quad 1.58141 .3473$

$-0.7014-1.0377 \quad 0.1290 \quad 0.5101$

EASE IMPEDAHCE TENSOR:

FFED-EX: 0.906 PRED-EY: 0.944
NORMALIZED TELLURIC TRANSFER TENSOR

$0.9261-0.0740 \quad 0.2094-0.0065$

0.20210 .12050 .5506

REMOTE TELLURIC TENSOR:

PRED-EX: 0.965 PREC-EY: 0.815

JEMEZ-BACA LOCATION (REMOTE)

JE-EI1-A

PERIOD: 20

AXIS: 1

COMPENSATEO TELLURIC

ELLIPSE PARAMETERS

COUHTER-CLOCKHISE ROTATIOH

MA JOP AKIS: 0.977

MIHOR AKIS: 0.627

AZIIUTH: 19.991

CLOCKWISE ROTATIOH

MAJOR AXIS: 1.059

MINOR AXIS: 0.290

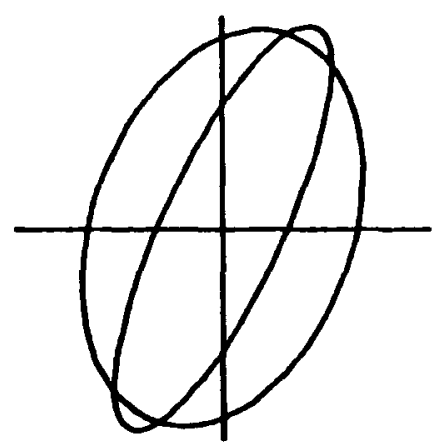


IHFUT IMPEDANCE TEHSOR

$\begin{array}{llll}-0.2914 & -0.6041 & 1.1827 & 1.1066\end{array}$

$\begin{array}{lllll}-0.0603 & -1.5337 & 0.2216 & 0.4703\end{array}$

BASE IMPEDAHCE TENSOR:

FRED-EY: 0.906 PRED-EY:

0.944
NORHALIZED TELLURIC TRANSFER TEHSOR

$\begin{array}{llll}0.7239 & -0.0241 & 0.2832 & 0.0989\end{array}$

$\begin{array}{llll}0.2188 & 0.0786 & 0.7887 & 0.1813\end{array}$

REMOTE TELLURIC TENSOR:

PRED-EY: 0.937 PRED-EY: 0.965

JEHEZ-EACA LOCATION (REHOTE)

$J \mathrm{E}-\mathrm{B} 12-\mathrm{A}$

FEFIUO: 20

AYIS: 1

COHFEHSATED TELLURIC

ELLIFSE FARAMETERS

COUHTEF-CLDCKWISE ROTATIOH

MAJUR AXIS: 0.946

HINOP ANIS: 0.563

A?IHUTH: $\quad 69.399$

CLOCKWISE ROTATIOH

MAJIJR GKIS: 1.126

HINOP GXIS: 0.416

ADIMUTH: $4 \dot{2} .205$

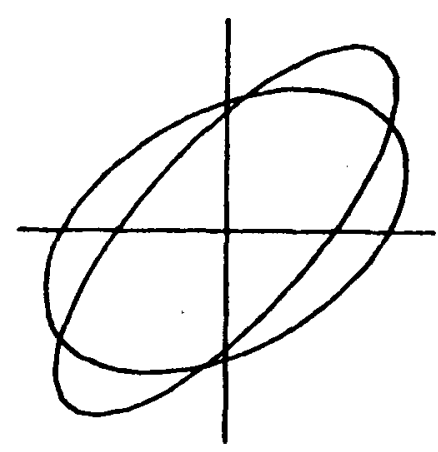

IHFUT IMFEDAHCE TENSOR

0.917E - $0.09190 .7250-0.0640$

$-0.3668-0.9762-0.0879-0.2922$

HORMAL IZED TELLURIC TRANSFER TEMSOR

$0.2280-0.2306-0.26110 .3192$

$\begin{array}{llll}-0.1202 & -0.0646 & 0.4247 & 0.1927\end{array}$

BÁSE IMFEDANHCE TEHSOR:

FFE[T-EX: U. GOE FRE[I-EY: 0.944

REMOTE TRLLURIC TENSOR:

PRED-EX: 0.732 PRED-EY: 0.778

JEMEZ-EACA LOCATIOH (REMOTE)

JE - E $13-A$

FERIOD: 29

Aि:1S: 1

COMPEHSATED TELLURIC

ELLIFSE FÂRAMETEPS

COUHTER-CLOCKWISE ROTATIOH

MAGJUR AYYIS: 0.623

HIHIT GEIS: 0.300

GEIMUTH: -42.838

CLUCKWISE ROTATIOH

HÁ JUR AXIS: O.55?

MIHUR AÝ1S: 0.485

AZIMUTH: $2 \dot{0.805}$

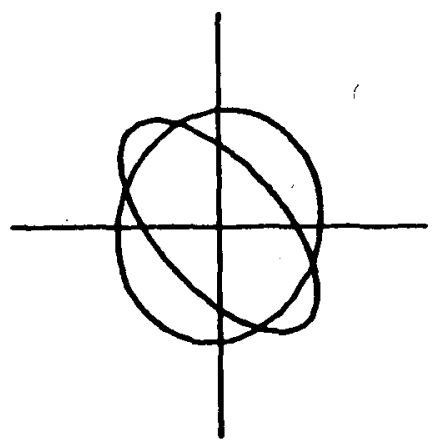


IHPUT INPEOAHCE TENSOR

$\begin{array}{lllll}-0.24 .3 & 0.4942 & 0.1219 & 0.2403\end{array}$

$0.0183-1.3397$

$0.0788-0.1827$

BASE IMPEDAHCE TENSOR:

FREL-EX: $\bar{\theta} .986$

PRED-EY: 0.944
NORMALIZEO TELLURIC TRANSFER TENSOR

$0.1145 \quad 0.0374-0.0781-0.2345$

$\begin{array}{llll}-0.0329 & -0.0827 & 0.4179 & 0.4294\end{array}$

REMOTE-TELLURIC TEHSOR:

PRED-EX: 0.206 PRED-EY: 0.304

JEMEZ-EACA LOCATIOH (REMOTE)

JE-E14-A

FERIOUL: 20

HิXIS: 1

COHFENSATED TELLURIC

ELLIFSE FARAMETERS

COUHTER-CLUCKHISE ROTATIOH

MAJUP AKIS: 0.653

MIHOF AKIS: 0.150

AZIHUTH: -60.033

CLGEKWISE POTATIOH

MAJOR AXIS: G. EEG

MINDP AKIS: 0.022

ALINUTH: -75.495

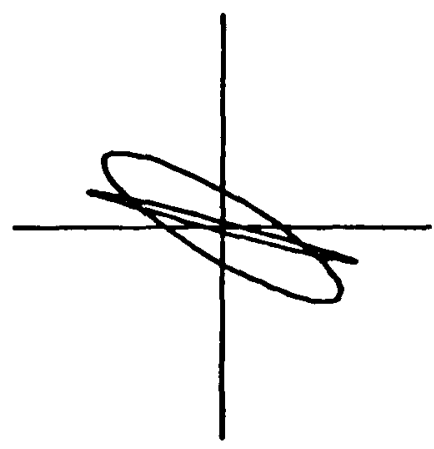

IHFUT IMPEDANCE TENSOR

$$
0.62790 .1693 \quad 0.1780 \quad 0.0991
$$

NORMALIZED TELLURIC TRAHSFER TENSOR

$-0.1371-0.1975-0.2201-0.1905$

$0.0877-0.0250-0.2521$

B. 1458

BHSE IPFEDAHCE TEHSOR:

FPED-EK: 6.9Q6 PRED-EY: 0.944

REMOTE TELLURIC TEHSOR:

PRED-EX: 0.321 PRED-EY: 0.094

JEHEZ-PACA LOCATIOH (REMOTE)

JE-E $15-A$

PEFIOC: 29

ARIS: 1

COMPEHSATED TELLURIC

ELLIPSE PARAAIETEPS

COUNTER-CLOCKHISE ROTATIOH

MAJOR AYIS: 0.311

MIHUR ÂIS: 0.131

A2IHUTH: $\quad-27.665$

CLOCYHISE POTATIOH

MGJOF AXIS: 0.342

MIHOR FXIS: 0.013

AZIMUTH: -18.151

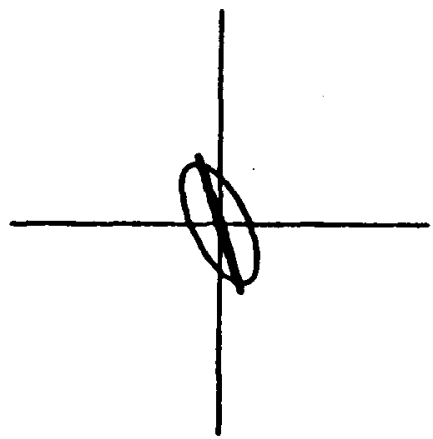


IHPUT IMPEDANCE TENSOR

$0.1886-0.41120 .0942-0.0365$

- घี. $3208-1.2652-0.1126-0.2924$

BASE IMFEOAHCE TEHSOR:

PREQ-EX: 0.906 PRED-EY: 0.944

JEMEZ-BACA LOCATION (REAOTE)

$J E-B 16-A$

PERIOD: $2 B$

AXIS: 1

CONPEHSATED TELLURIC

ELLIPSE PARAMETERS

COUHTER-CLOCYWISE ROTATIOH

HÂJOF AYIS: 0.632

MINOR AYIS: 0.101

AZIHUTH: 76.889

CLOCKWISE ROTATIOH

MAJOR FYIS: B. EG9

MIHOR AYIS: B.159

ARIMUTH: 72.7P1
HORHALIZED TELLURIC TRANSFER TEMSOR

$\begin{array}{rlll}0.0182 & -0.0413 & 0.0704 & 0.1897 \\ -0.1281-0.0569 & 0.5015 & 0.2987\end{array}$

REMOTE TELLURIC TENSOR:

PRED-EX: 0.297 PFEQ-EY: 0.452

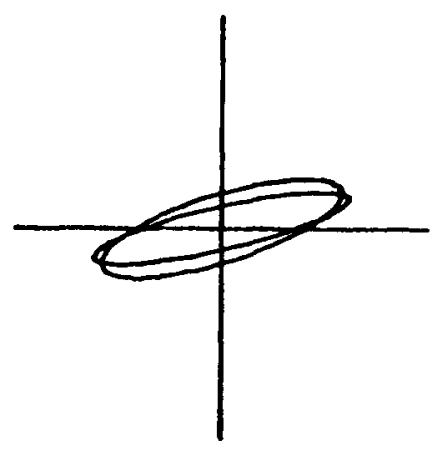

MORMALIZED TELLURIC TRANSFER TEHSOR

$\begin{array}{llll}0.4038 & -0.0186 & 0.1603 & 0.2857\end{array}$

$\begin{array}{llll}0.2564 & -0.0457 & 0.7875 & 0.2523\end{array}$

REMOTE TELLURIC TEHSOR:

EASE IMPEDAHCE TEHSOR:

0.3331

FFED-E:: 0.906 PRED-EY: 0.944

JEMEZ-BACA LOCATION (REMOTE)

IE-B17-A

PERIOD: 20

A.:IIS: 1

COHFEHSATED TELLURIC

ELLIFSE FÂFAIHETERS

COUHTEP-CLOCKHISE ROTATIOH

MÂJOR AKIS: 0.756

MIHOR GKIS: $\bar{\theta} .115$

ACIMUTH: $\quad 38.871$

CLOCKWISE ROTATIOH

MAJOR AYYIS: 1.130

MIHOF AYIS: 0.428

AZIIUTH: SP.G15

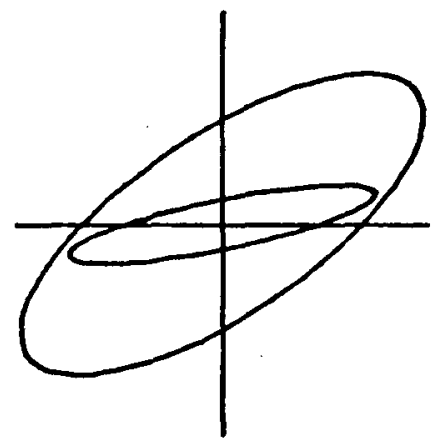


Normalized Telluric Ellipses (Unit Amplitude Circularly

Polarized Magnetic Field)

$$
T=30 \text { seconds }
$$

INPUT IMFEDAHCE TEHSOR

$-0.5122 \quad 0.40830 .2988$

$-0.5352-1.5022-0.8519$

0.2825

0. 2831

BGEE IHFEDAHCE TEHSOR:

FFE $1-E X:$ B. 9 PFE[-EY: 0.915
NORMALIZEO TELLURIC TRAHSFER TENSOR

$0.2251-0.0053 \quad 0.0403-0.3565$

$\begin{array}{llll}-0.2203 & 0.4396 & 0.7891 & 0.3745\end{array}$

REIHOTE TELLURIC TENSOR: PRED-EX: 0.585 PREC-EY: 0.701

IEHEZ-BACA LOCATIOH (REMOTE?

$J E-E \hat{Z}-\hat{A}$

PEFIOR: 30

A.:15: 1

COMPEHSATEO TELLURIC

ELLIFSE FARAHETERS

COUHTER-CLOCKHISE ROTATIOH

NA JOR AYIS: 1.386

MIHUT AYIS: 9.536

HEINUTH: -\$.117

CLOCKWISE POTATION

HAJOR AYIS: 0.382

MIHOR AXIS: 9.139

AZIIIUTH: -88.198

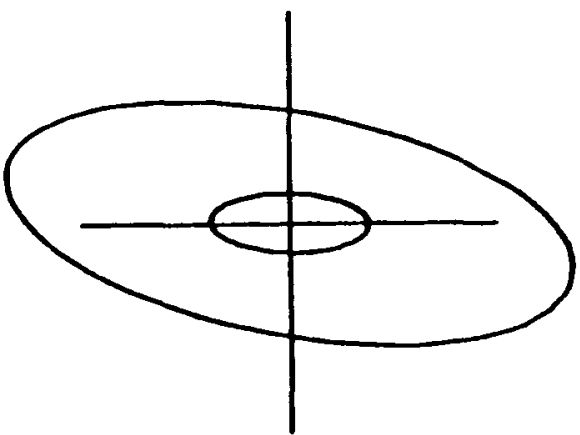

IHFUT IMPEDANCE TENSOR

$\begin{array}{rrrr}-0.5274 & -0.0621 & 0.7485 & 0.8199 \\ -1.1434-2.0075 & -0.6936 & 1.1314\end{array}$

MORMAL IZED TELLURIC TRANSFER TENSOR

$-1.1434-2.0075-0.6936$

1.1314

0.6074

Q. $02770.2283-0.1802$

$\begin{array}{llll}0.1696 & 0.7068 & 1.2203 & 0.3347\end{array}$

EASE IMPECHACE TENSOP:

FFE[I-EY: Q.9 FRE[-EY: B.915

REMOTE TELLURIC TEHSOR:

PRED-EK: 0.8 PRED-EY: 0.821

JEMEZ-EACA LOCATIOH (REHOTE)

JE-B $\bar{S}-\hat{A}$

FEDICI: 30

A

COHPENSATEO TELLIJPIC ELLIFSE PAFAHETEPS

COUNTEF-CLOCKNISE ROTATIOI

MHJOF A.OIS: 1.945

MINISP AXIS: 0.892

A?IMUTH: $\quad 83.312$

CLOCY.WIEE POTATIOH

MÚJUR GYIS: 9.849

MINOF G\%IS: 9.139

G?IIUTH: 57.431 
IHPUT IMPEDANCE TEHSOR

$-1.0984-0.14350 .6465$

1.1372

NORMALIZED TELLURIC TRANSFER TENSOR

$-0.5472-1.8747-0.3645$

0.2133

0.6908

0.1908

$0.4810-0.3698$

EHSE IMPEDAHIIEE TEHSOR:

FREO-E : : 0.9 PRED-EY: 0.915

REMOTE TELLURIC TEHSOR:

PRED-EX: 0.934 PRED-EY: 0.985

JEHEZ-EGCA LOCATION (PEMOTE) JE-E:T-A

PEFIOQ: 30

A\%1S: 1

COHPEHEATED TELLURIC

ELLIPEE PARAMETERS

COUNTEP-CLOCKHISE ROTATIOH

MAJIJR AYIS: 1.344

MIHIT ARIS: 1.293

AZIHUTH: $\quad 5.3 .292$

CLUCKHISE FOTATIÖH

MAJIJR GYIS: 0.946

MINGP A\%IS:

AZIHUTH:

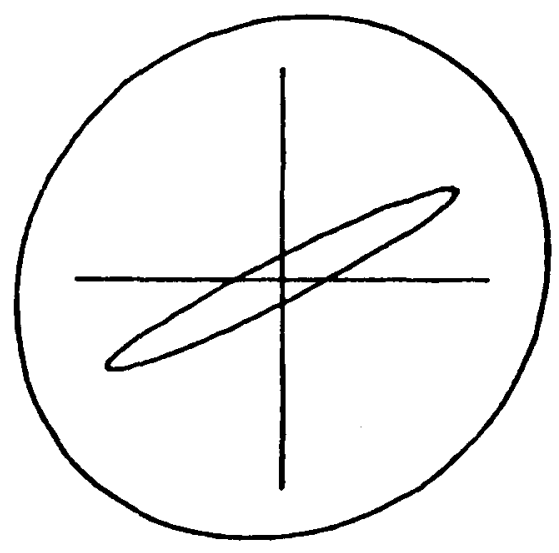

IIFEUT IHPEDAHCE TEHSOR.

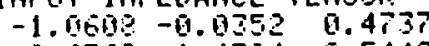

$-0.560-1.4784-50.5448$

0.9228

9.0775

EHSE IHFEDAHYE TENSOR:

FFE[1-E: B.

PFE[I-EY:
MORIAALIZED TELLURIC TRAMSFER TEMSOR

$0.54 \dot{0} \quad 0.1739 \quad 0.4245-0.3972$

$-0.1810$

0.2410

0. 8270

0.3182

REMOTE TELLURIC TEHSOR:

PREO-EY: 0.846 PRED-EY: 0.9

JEMEZ-EÁCA LJCATIOH (REMOTE)

$J E-E: \hat{Y}-\hat{M}$

FEP IOO: 30

H.XIS: 1

COMFENEATED TELLURIC

ELLIFSE PGFAMETERS

COUHTER-CLOCKUIISE POTATIOH

MAJJP AYYIS: 1.225

MILITR HUIS: 1.GEZ

A2IHUTH: 5E.977

CLOCYWISE POTATIOH

MHJOR AOIS: $9.6 \in 3$

HIHOR HEIS: 9.075

GZIMUTH: 64.965

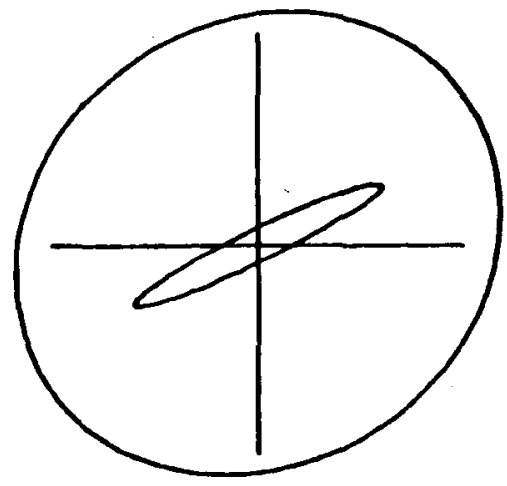


INFIIT IMFERAHCE TEHSOR $-3.6558-1.4509 \quad 2.6943 \quad 4.5658$ $-6.6169-1.6525-0.2167$

0.0724

BASE IMFEDANCE TENSOR:

FFER-E : 1.9 PRED-EY: 0.915

IEIIE- - BACG LOCATION (REMOTE)

JE -E:1Ci-M

FEFINT: 30

AXIS: 1

COMFEHSATED TELLURIC

ELLIPSE PAFGHETERS

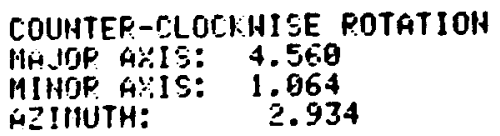

CLOCKHISE PRTATIOH

MATJOP A\%IS: 2.434

14INOR GXIS:

AEIMIJTH:

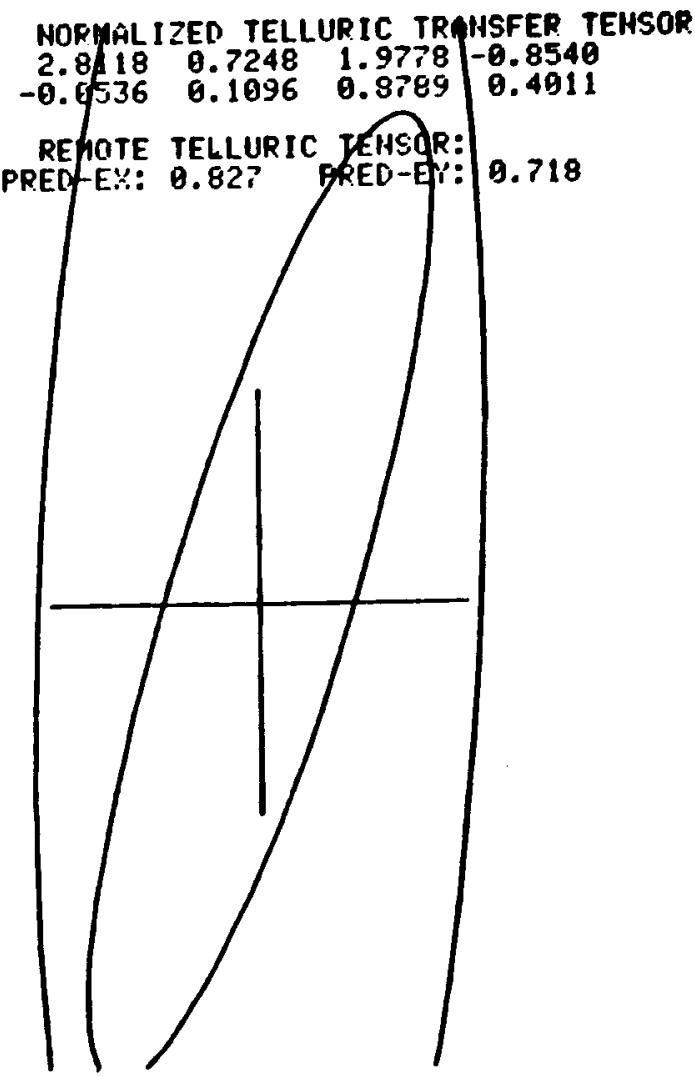

INPUT IMFECANCE TEHSOR

$\begin{array}{llll}-0.3351 & -0.3118 & 1.4215 & 1.3354 \\ -0.40 .55 & -0.6179 & 0.0668 & 0.4049\end{array}$

EASE IMFELAHCE TENSOR:

FFE[D-EX: 0.9 PRED-EY: 0.315
HORIAAL IZEO TELLUPIC TRAHSFER TENSOR

$1.067 ?-0.03340 .2517-0.0102$

G.1826 $0.1310 \quad 0.4269 \quad 0.9596$

FEHOTE TELLURIC TENSOP:

FRED-EY: 0.966 PPET-EY: 0.684

JEMEZ-EAIA LOCATIOH (PEMOTE) JE:-E11-A

FEFIOIS: 30

AXIS: 1

COHFEHSATED TELLURIC

ELLIPSE FARAAIIETERS

CDUNTEP-CLDCKWISE ROTATION

MAJJF: A.YIS: 1.139

HIHOF A.XIE: 0.592

HEIHUTH: 14.349

CLOCHWISE POTATIOH

MAJUF AXIS: 1.139

MIHOE AXIS: 0.298

GZIHUTH: $\quad 16.270$

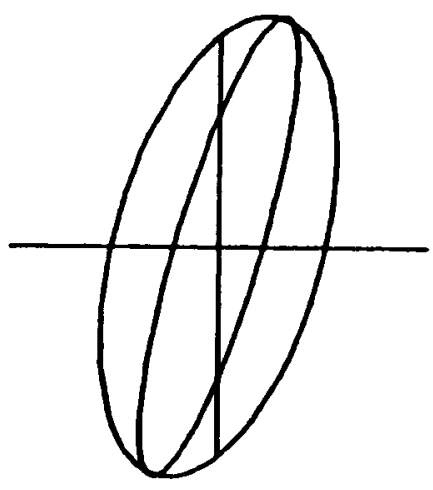


IMPUT IMPEDANCE TEHSOR.

$-0.2441-9.49640 .9387$

1.2619

0.3968

BASE IMPEDANCE TENSOR:

FFET-EX: 0.9 PFED-EY: 0.915
NORMALIZED TELLURIC TRANSFER TENSOR

$0.8523 \quad 0.1251 \quad 0.2868 \quad 0.0977$

$0.14010 .16720 .6578 \quad 0.2903$

REMOTE TELLURIC TENSOR:

PRED-EX: 0.834 PRED-EY: 0.96

JEME?-BACA LOCATION (REMOTE)

JE-B12-A

FERIOT: 38

AX15: 1

COMEENSATED TELLURIC

ELLIFSE PARAMETERS

COUNTER-CLOCKHISE ROTATIOH

MAJOP AYIS: 1.004

MIHOR A\%IS: $\quad 0.645$

AZIMUTH: 42.345

CLOCKWISE ROTATION

MG.JOR AXIS: 1.066

MIHOR AXIS: 0.336

AZIMUTH: $27.31 B$

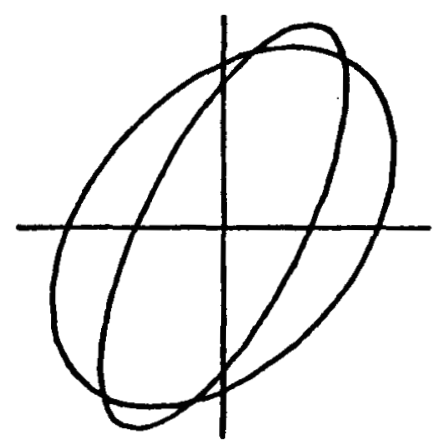

INFUT IMPEDANCE TENSOR

$0.4931-0.1114 \quad 0.8524$

0.1799

HORMALIZED TELLURIC TRANSFER TEHSOR

$-0.2364-0.78900-0.5661$

0.0096

$\begin{array}{rrr}0.2190 & 0.2195 & 0.3971\end{array}$

0.2341

PASE IMPECAHCE TEHSOR:

FFET,-EY: 6.9 PFED-EY: 0.915

REMOTE TELLURIC TENSOR:

PRED-EX: 0.82 PRED-EY: 0.859

JEMEZ-BACG LOCATION (REMOTE)

JR-E13-A

PERIOC: 30

AXIS: 1

COMPENSGTED TELLURIC

ELLIPSE PAFAMETERS

COUNTER-CLOCKWISE POTATIOH

MA.JOR AYIS: 9.869

MIHDR AXIS: 9.986

AZIMUTH: -59.992

CLOCKHISE POTATIOH

MAJOR A.UIS: 8.644

MIHOP AYIS: 0.176

AZIMUTH: Z.525

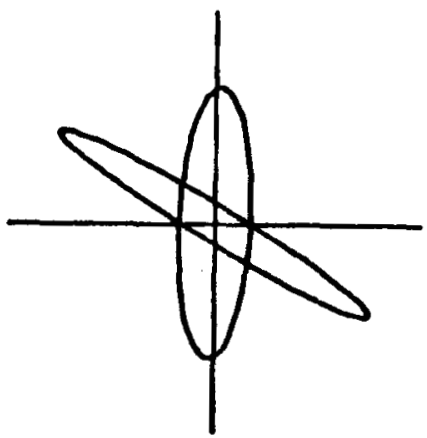


INFUT IHPEDAHCE TENSOR

- $5.1400 .7999 \quad 0.5195 \quad 0.9135$

$0.1=0-0.5463-0.3630-0.3620$

EAEE IHIFEOAHCE TEHSOR:

FFE[I-EX:

JEMEZ-EACA LOCATIOH (REMOTE)

IE $-E$ 1 $14-\hat{A}$

FEFIOC: $3 B$

A.:15: 1

COHFEHSATEO TELLURIC

ELLIPSE FARANETERS

COUHTEP-CLOCKHISE ROTATIOH

MAJUF AXIS: 1. DE9

MINUF Aris: 0.070

A2IMUTH: $\quad-31.857$

CLOCKHISE POTATION

MAJOP H\%1S: 0.469

MINOP AYIS: $9.05 ?$

MCIMUTH: -16.584
MORMALIZEO TELLURIC TRAHSFER TEHSOR

$0.5550 \quad 0.1526-0.2548-0.3648$

$\begin{array}{llll}-0.2808 & 0.0004 & 0.1447 & 0.2784\end{array}$

REMOTE TELLURIC TENSOR:

PRED-EX: 0.554 PRED-EY: 0.256
IMPUT IMPEDAICE TENSOR

$\begin{array}{llllll}0.1968 & 0.5414 & 0.5437 & 0.0471\end{array}$

-0.2725 0.0451-0.3727 0.0609

Base IMPEDANCE TENSOR:

FRET-EK: 0.9 PRED-EY: 0.915
MORMALIZED TELLURIC TRAMSFER TEHSOR

$0.2288-0.1923-0.2859-0.1334$

$\begin{array}{llll}-0.1211 & 0.1676 & 0.0880 & -0.1230\end{array}$

REMOTE TELLURIC TENSOR:

PRED-EX: 0.362 PRED-EY: 0.176

JEME2-BACA LOCATIOH (REMOTE)

JE -E15-A

FEP IOO: 30

AxIs: 1

COMPENSATED TELLURIC

ELLIP'SE PARAMETERS

COUHTEP-CLOCKHISE POTATIOH

MÂJOR AXIS: 0.635

MINTR AYIS: 0.147

ALIHIJT: $\quad-19.366$

CLOCKHISE POTATIOH

MAJOR AKIS: 0.266

MIIIOP A.XIS: 0.114

A2IHUTH: $\quad-73.309$

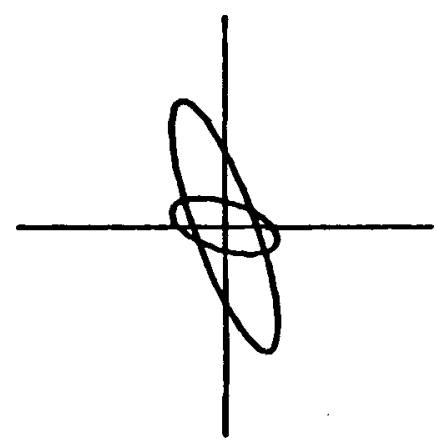


INPUT INPEDAHCE TEHSOR

$0.3458-0.4446 \quad 0.1680-0.1802$

$-0.4062-0.8633-0.4316-0.0984$

BASE IMFEDAHCE TEHSOR:

FFECI-EY: 0.9 PFED-EY: 0.915
MORMALIZED TELLURIC TRAHSFER TEHSOR

$\begin{array}{llll}-0.0547 & -0.1349 & 0.0383 & 0.3061\end{array}$

$\begin{array}{llll}-0.2053 & 0.1291 & 0.4917 & 0.1770\end{array}$

REMOTE PELLURIC TEHSOR:

PRED-EX: 0.457 PRED-EY: 0.455
JEMEZ-EACA LOCATION (PEMOTE)

JE:-E:16-A

PEFIIIL: 35

4.15: 1

COMPEHSATEO TELLURIC

ELLIFGE PARAMETEPS

COUHTEP-CLOCKWISE ROTATIOH

MAJUF AXIS: 0.734

HIHOF AYIS: 0.313

AिLIMUTH: $8 \dot{2} .2 \epsilon 5$

CLOCKHISE POTATION

MAJUR AXIS: 0.426

MIHOF A.YIS: 0.268

ALIMUTH: $\quad 48.010$

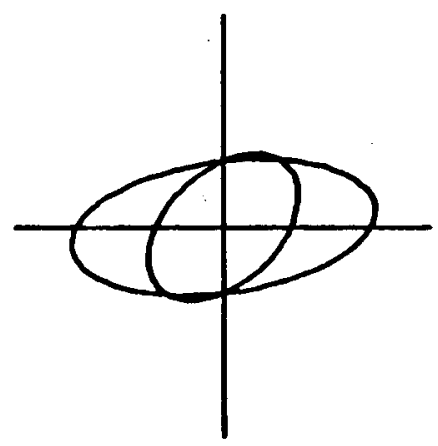

INPUT IMFEDAHCE TENSOR

$0.1169-0.2747 \quad 0.4040 \quad 0.2861$

$-6.5589-1.1913-0.6100 \quad 0.0426$

EÂSE IMPEDAHCE TENSOR:

FFEL-EX: 0.9 FRED-EY: 0.915
MORMALIZED TELLURIC TRAHSFER TENSOR

$0.2673-0.0457 \quad 0.06110 .1517$

$\begin{array}{llll}-0.2197 & 0.2527 & 0.6748 & 0.2480\end{array}$

REMOTE TELLURIC TENSOR:

PRED-EX: 0.547 PRED-EY: 0.689

JEHEZ-BACA LOCATIOH (REHOTE)

JE:-E 1 T $-\hat{H}$

FER IOUD: 30

AKIS: 1

COMPENSATED TELLURIC ELLIFSE FARAMETERS

COUHTER-CLOCKHISE POTATIOH

MAJOP AXIS: 1.640

MIHIR AKIS: 0.110

AZIMUTH: -87.866

CLOCKHISE ROTATIOH

MAJOR AXIS: 0.490

HINDR AKIS:

A?IMUTH: 42.983

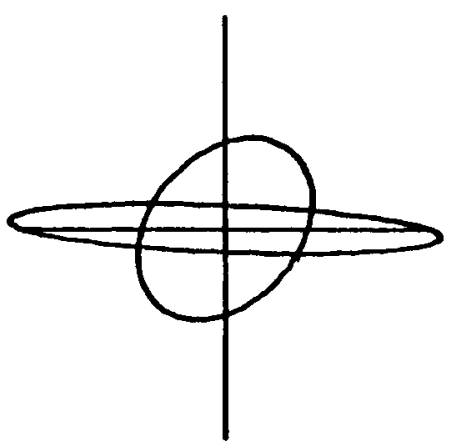




\section{SECTION B}

Part 1

Compensated Telluric Ellipses (20 ohm-m)

$T=20$ seconds

INFUT IHFEDANCE TEHSOR

$\begin{array}{rrr}-0.7814 & 0.1217 & 0.7029 \\ -0.3324 & -3.4264 & -0.7648\end{array}$

0.6352

$0.957 ?$

EASE IHFEDAHCE TEHSOR:

FFED-EX: $\overline{0} .906$

PFEO-EY:
MORMALIZED TELLURIC TRANSFER TENSOR

$0.4232-0.02140 .2086-0.2856$

$0.0610 \quad 0.5447 \quad 1.2044 \quad 0.9626$

REMOTE TELLURIC TENSOR:

PREO-EX: 0.623 PREO-EY: 0.722

JEHEZ-BACA LOCATIOH (REMOTE)

JB-E: $5-A$

FEFIOR: 20

AXIS: 1

FHÁSE-CORFECTED

CONHFEHSATED TELLURIC

ELLIPSE PARAMETEFS

MA.JUF AXIS: 1.236

MINOF AXIS: 0.494

A2IIIUTH:

77.889

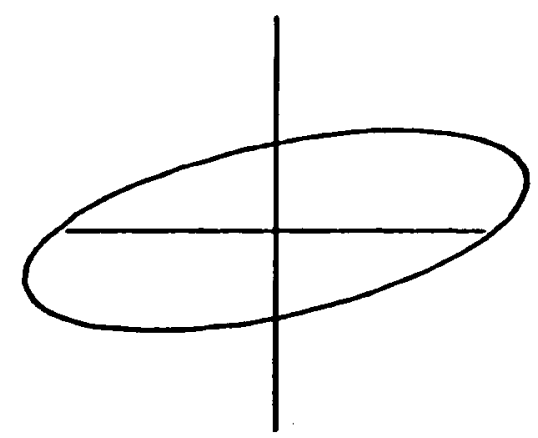

IHPUT IMPEDAHCE TEHSOR

$\begin{array}{lll}-0.8300 & 1.1612 & 0.5336\end{array}$

$-1.7391-4.4630-1.3764$

FFED-EY: B. 90 PRED-EY:

PRED-EY: 0.944
HORMALIZED TELLURIC TRANSFER TEMSOR

0.3164

0.2119 $\begin{array}{rrrr}0.2688 & -0.0687 & -0.1082 & -0.6389 \\ -0.3682 & 0.5023 & 1.9613 & 0.8614\end{array}$

T.9613

REMOTE TELLURIC TENSOR:

FRED-EY: 0.757 PREO-EY: 0.83

JEIAEZ-BACA LOCATIOH (REMOTE)

JE-E $\vec{T}-\hat{A}$

FERIO0: 20

Ar.IS: 1

PHASE-COPRECTED

COHPEHSATED TELLURIC

ELLIPSE PÂRAMETEPS

MÂJOR Â:IS: 2.002

MINDF AYIS: 0.243

AEINUTH: $\quad-85.464$

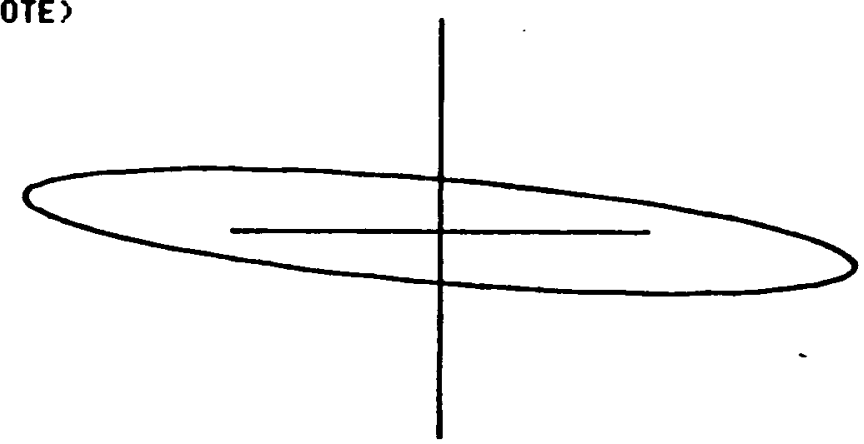


INPUT IMPEDANCE TENSOR

$\begin{array}{lll}-1.1419 & 0.3316 \quad 0.8351\end{array}$

$-1.2579-2.8144-0.1494$

1.0871

0.1886

BASE IMPEDAHCE TEHSOR:

PRED-EK: 0.906

PRE O-EY: 0.944
NORMALIZED TELLURIC TRANSFER TENSOR

$0.60780 .079 \% 0.2563-0.4668$

$\begin{array}{llll}0.0124 & 0.1069 & 1.2878 & 0.4922\end{array}$

REMOTE TELLURIC TENSOR:

PRED-EX: 0.939 PRED-EY: 0.989

JEMEZ-EACA LOCATION (REMOTE)

JB-E:-4

PERIOD: 28

AXIS: 1

PHASE-CORPECTEO

COMPENSATED TELLURIC

ELLIPSE PARAIIETEFS

MAJOR AXIS:

MIHOR GXIS:

1.321

AZLIMUTH:

0.595

75.555

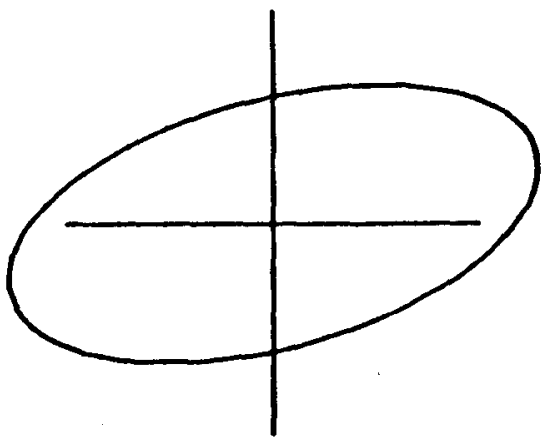

INPUT IMPEDANCE TENSOR

$\begin{array}{llll}-1.0942 & 0.1018 & 0.4511 & 0.6022\end{array}$

HORMALIZEO TELLUR.IC TRANSFER TENSOR

$-1.1359-2.2656-0.2372-0.0536$

$-6.0920$

0.0478

$0.3138-0.3782$

BASE IMPEOAHCE TEHSOR:

REMOTE TELLURIC TEHSOR:

FFED-EX: B̈. 9065 PFED-EY: 0.944

PRED-EK: 3.829

PREO-EY: 0.956

JEME?-EACA LOCATION CREMOTE:

$\mathrm{JE}-\mathrm{E} \overline{\mathrm{g}}-\mathrm{A}$

FERIÓD: 20

AX1S: 1

PHASE-CORRECTED

COMFENSATEO TELLIJRIC

ELLIPSE PARAMETERS

$\begin{array}{ll}\text { MAJOR AYIS: } & 1.129 \\ \text { MINOR A\%IS: } & 0.345 \\ \text { ADIMUTH: } & 73.649\end{array}$

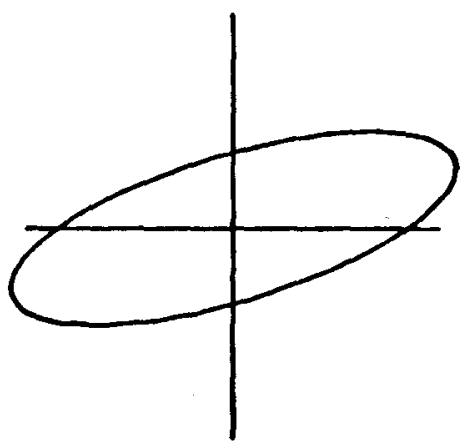


INPUT IMPEDAHCE TENSOR $\begin{array}{lll}-3.1935 & 0.4992 & 2.6851 \\ -0.9609 & -2.5465 & -0.3867\end{array}$

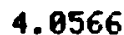

4.0566
0.3539

BASE IMPEDAHCE TENSOR:

F.PECI-E:: 0.986

PRED-EY:

0.944
HORMALIZED TELLURIC TRANSFER TEMSOR

$\begin{array}{llll}2.1966 & 0.4590 & 0.2520 & -1.1677\end{array}$

$\begin{array}{llll}-0.0104 & 0.2342 & 1.1107 & 0.4998\end{array}$

REMOTE TELLURTC TEASOR:

PFED-EX: g.\$66 PRED-EY: 0.706

JEMEZ-BACA LOCATIOH (REMOTE)

JE-E1B-A

PEFIOD: 20

AYYIS: 1

PHÂSE-COPPECTEO

COHPENSGTEO TELLURIC

ELLIFSE PAPAMHETEFS

MAJDR AYIS: 2.317

HINOR AH:IS: 1.014

AZIMUTH: $\quad 12.599$

IHPUT IMPEDANCE TENSOR

$-9.2914-6.6041 \quad 1.1827 \quad 1.1066$

$\begin{array}{llll}-0.0603 & -1.5337 & 0.2216 & 0.4703\end{array}$

EASE IMFE[MHCE TEHSOR:

FFECI-EX: U1.9TE PFER-EY: 0.944
MORHAL IZED TELLURIC TRAHSFER TEHSOR

$\begin{array}{llll}0.7239 & -0.0241 & 0.2832 & 0.0989 \\ 0.2188 & 0.0786 & 0.7887 & 0.1813\end{array}$

REMOTE TELLURIC TENSOR:

PRED-EY: 0.937 PRED-EY: 0.965

JEMEZ-EACA LQCATIOH (PEMOTE:

$\mathrm{J} \mathrm{T}-\mathrm{B} 1 \mathrm{2}-\hat{\mathrm{A}}$

FERIUO: 20

Aris: 1

PHASE-CORRECTEO

EOMFEHSATED TELLURIC

ELLIFSE FARAMETEPS

MÂ.JOP Aि\&IS: 1.010

MIINOF AOIS: 9.504

AEIMUTH:

47.457

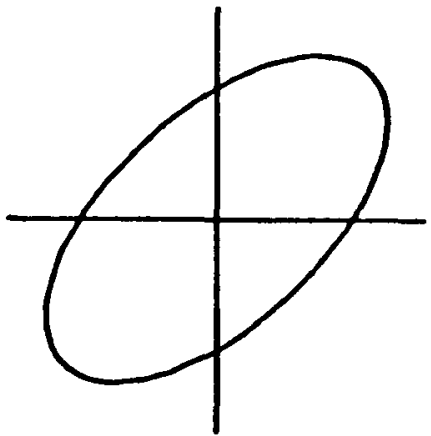


IHPUT IMPEDAHCE TENSOR

$\begin{array}{lll}-0.3414-0.3207 & 1.5814 & 1.3473 \\ -0.7014-1.0377 & 0.1290 & 0.5101\end{array}$

BASE IMFEOANCE TENSOR:

PRED-EX: 0.986

PRED-EY: 0.944
NORMALIZED TELLURIC TRANSFER TEMSOR

$\begin{array}{rrrr}0.9261 & -5.0740 & 0.2094 & -0.0065 \\ 0.2021 & 0.1295 & 0.5500 & 0.1063\end{array}$

REMOTE TELLURIC TEHSOR:

FRED-EX: 0.965 PRED-EY: 0.815

JEMEZ-EACA LOCATIOH (REMOTE)

JE:E $11-\hat{A}$

FERIO0: 20

AYIS: 1

PHASE-CORPECTED

COMPEHSATEO TELLURIC

ELLIFSE PARAHETERS

MAJUP AYYIS: 1.917

MIHOF AYIS: 0.459

AZIMUTH: 23.642

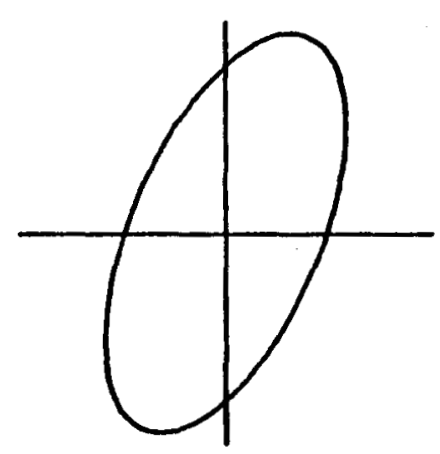

IHPUT IMPEDAHCE TENSOR

$0.9176-0.09190 .7250-0.0040$

$-0.3668-0.9762-0.0879-0.2922$

PASE INPEDANCE TENSOR:

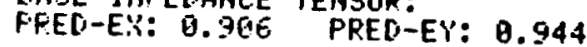

MORMALIZED TELLURIC TRANSFER TEMSOR

$0.2289-0.2306-0.26110 .3192$

$\begin{array}{llll}-0.1202 & -0.0646 & 0.4247 & 0.1927\end{array}$

REMOTE TELLURIC TEHSOR:

FRED-EY: 0.732 PRED-EY: 0.778
JEMEE-EACA LOCATIOH (REMOTE)

$J E-E 13-\hat{A}$

FEFIOT: 20

AxIS: 1

PHASE-CORRECTED

COHFEMSATED TELLURIC

ELLIFSE FARAGIIETERS

MA.JOR AXIS:

MIHOR G.:IS:

0.548

HZIMUTH:

0.119

$-52.554$

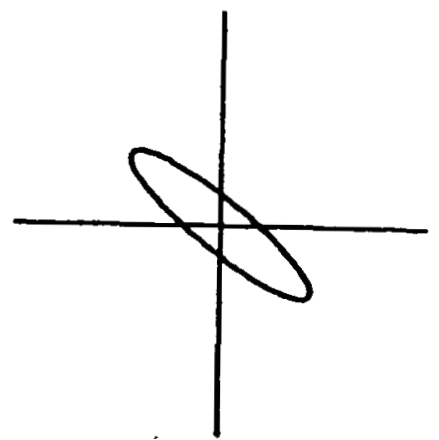


INPUT IMPEDAHCE TENSOR

$\begin{array}{llll}-0.2473 & 0.4942 & 0.1219 & 0.2403\end{array}$

B. $0183-1.33970 .0788-0.1827$

BASE IAPECAHCE TEHSOR:

FFE[-E:: 0.9日E) PREG-EY: 9.944
MORMALIZEO TELLURIC TRAHSFER TENSOR

$0.11450 .0374-0.0781-0.2345$

$\begin{array}{llll}-0.0329 & -0.0827 & 0.4179 & 0.4294\end{array}$

PEMOTE TELLURIC TEHSOR:

PREO-EK: 0.206 PPED-EY: 0.384

JEMEZ-EACA LOCATION (REHOTE)

IE-E 14-A

FEPIOL: 20

AिXIS: 1

PHASE-CORRECTED

CUHAEHSATEO TELLURIC

ELLIFSE PARAMETERS

MÁJDP ALIS: 0.429

MIHIOF A.:IS: 0.106

AEIHUTH: -?T.528

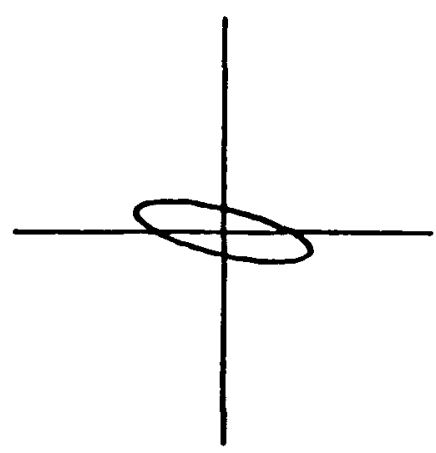

IHPUT IMPEDAHCE TEHSOR

G.6279 $0.1693 \quad 0.17890 .0991$

$-0.1371-0.1875-0.2200-0.1005$

BÂSE IMFEOAHHCE TENSOR:

PFE[1-E:: $0 . \overline{106}$ FRED-EY: 0.944
NORMAL IZED TELLURIC TRANSFER TEMSO

$0.0877-0.0250-0.25210 .1450$

$\begin{array}{llll}-0.1014 & 0.0378 & 0.1027 & 0.0159\end{array}$

REMOTE TELLURIC TENSOR:

PFE[-EY: 0.321 PRED-EY: 0.094

JEMEZ-EACA LOCATION (PEMOTE)

JE-B15-Aि

PEPIOL: 20

AX15: 1

PHASE-COFRETTEO

CÜMFENSATED TELLURIC

ELLIPSE PAFAMETERS

MAJJP ARIS: 0.298

MIHUF A\%IS: 0.055

AIIMUTH: -27.026

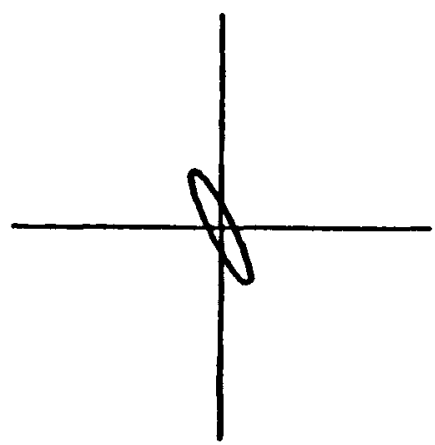


IHPUT IMPEDAMCE TENSOR

$0.1886-0.41120 .0942-0.0365$

$-0.3258-1.2552-0.1126-0.2924$

EASE IMPEOANCE TENSOR:

PFE[T-EK: E.906 FRED-EY: 0.944
WORMALIZED TELLURIC TRAHSFER TENSOR

$0.0182-0.0413 \quad 0.07040 .1897$

$\begin{array}{llll}-0.1281 & -0.0569 & 0.5015 & 0.2987\end{array}$

REMOTE TELLURIC TEHSOR:

FRED-EY: 0.297 PFED-EY: 0.452

JEHEZ-EACA LOCATIOH (REMOTE)

JE-E $1 \epsilon-\hat{A}$

FERIOT: 20

Aैं: IS: 1

PHASE-COREECTED

COMPEHSATED TELLURIC

ELLIFSE FARRAMTERS

MAJBR AX:IS:

MIHITE HAIS:

ÁZIHISTH:

0.522

0.035

82.952

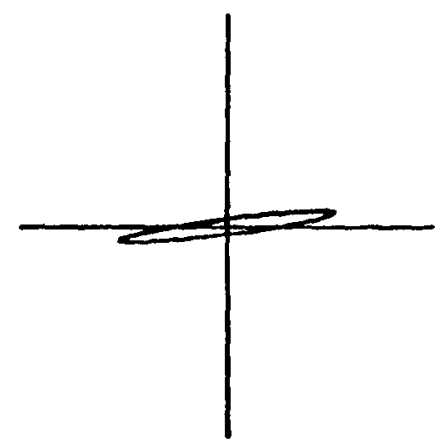

IHFUT IMPEDANCE TENSOR

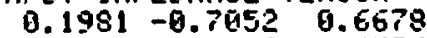

$\begin{array}{rl}0.8981-0.8052 & 0.6679 \\ -0.8463-1.6440 & 0.4776\end{array}$

0.6090

0.3331

BASE IMFEOANCE TENSOR:

PREV-E⿱口: 0.906

PRED-EY: 0.944
MORMAL IZED TELLURIC TRAHSFER TEHSOR

$\begin{array}{llll}0.4038 & -0.0186 & 0.1683 & 0.2857\end{array}$

$\begin{array}{llll}0.2564 & -0.0457 & 0.7875 & 0.2523\end{array}$

REMTTE TELLURIC TEHSOR:

PREO-EY: 0.849 PREC-EY: 0.831

JEMEZ-BACA LOCATIOH (REMOTE)

IE-EIT-A

FEFIOO: 20

A.:IS: 1

PHASE-CORRECTED COMPENSATEO TELLURIC

ELLIFSE PARAMETERS

MAJOR APIS: 0.881

HINOP A.XIS: 0.314

GZINUTH:

68.625

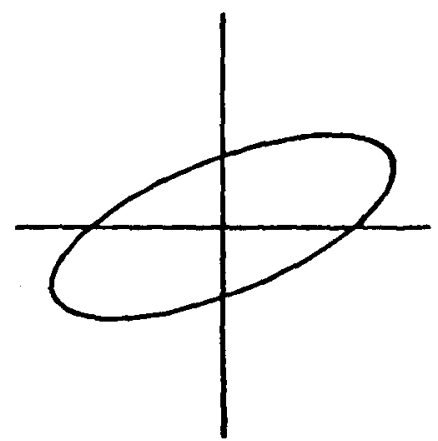


SECTION B (CON'T)

Part 2

Compensated Telluric Ellipses (20 ohm-m)

$T=30$ seconds

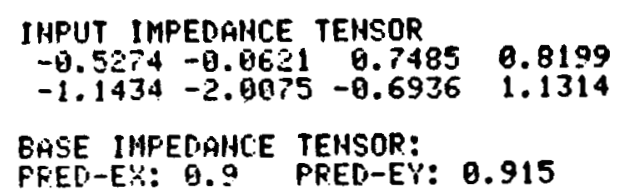

IHPUT IMPEDAHCE TEHSOR

$-0.5274-0.0621 \quad 0.7485$

FFEO-EY: 9.9
NORMALIZED TELLURIC TRANSFER TENSOR

$0.6074 \quad 0.02770 .2283-0.1802$

$0.1696 \quad 0.7068 \quad 1.2203 \quad 0.3347$

REMOTE TELLURIC TENSOR:

PRED-EX: 0.8 PRED-EY: 0.821

JEMEZ - EACA LOCATION (REMOTE)

$J E-E 5-A$

PERIOC: 30

AxIS: 1

PHASE-CORFECTED

COAPENSATED TELLURIC

ELLIPSE PARAMETERS

IAAJOP AXIS:

MIHUF GYIS:

AZIMUTH:

1. 289

9.549

72.584

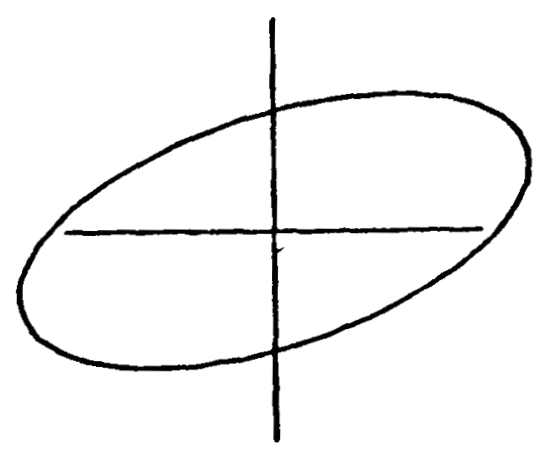

IHFUT IMPERANCE TENSOR $\begin{array}{rrrr}-0.5122 & 0.4083 & 0.2988 & 0.2825 \\ -0.5352 & -1.5022 & -0.8519 & 0.2831\end{array}$

BASE IHPETANCE TEHSOR:

FFE[1-EX: 0.9 PRED-EY: 0.915

NORMALIZED TELLURIC TRANSFER TENSOR

$0.2251-0.8063$

$-0.22030 .4396$

$0.0403-0.3565$

REMOTE TELLURIC TEHSOR:

0.3745

PRED-EY: 0.595 PRED-EY:

0.791

JEMEZ-BACA LOCATIOH (REMOTE)

$J E-E \bar{T}-\hat{A}$

FERIOL: 30

a,IS: 1

FHASE-CORPECTEO

COHFENSHTED TELLURIC

ELLIFSE FAPAMETERS

MAJUR AXIS: 9.820

HIHOR AXIS: 0.228

A. INUTH: -88.351

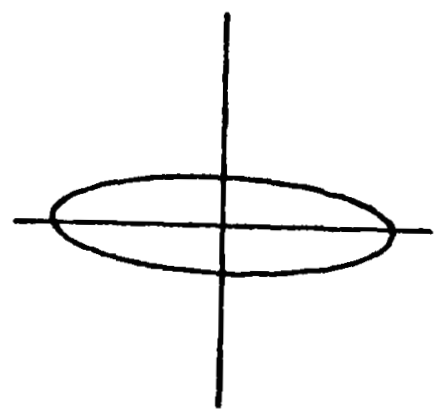


INPUT IMPEDANCE TENSOR

$\begin{array}{lll}-1.0984 & -0.1435 & 0.6465\end{array}$

$-0.5472-1.8747-0.3645$

1.1372

EASE IMPEDAHCE TEHSOR:

PFED-EX: 0.9 FRED-EY: 0.915

JEMEZ-BACA LOCATIOH (REMOTE)

JE-ES-A

PEFION: 30

G.:IS: 1

PHASE-CORRECTED

COHFEHSATEG TELLURIC

ELLIPSE PARAMETERS

MAUTE AYIS: 1.103

MINIJP AYIS: 0.613

A2IHUTH: 51.093
MORMAL IZED TELLURIC TRANSFER TENSOR

$0.6908 \quad 0.1900 \quad 0.4819-0.3698$

$\begin{array}{cccc}-0.0586 & 0.2238 & 0.9380 & 0.5142\end{array}$

REMOTE TELLURIC TENSOR:

PRED-EX: 0.934 PRED-EY: 0.985

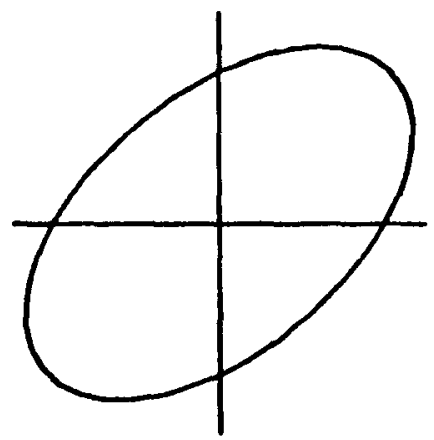

IHPUT IMPEDANCE TENSOR

$-1.0608-0.0352 \quad 0.4737$

0.9228

0.0775

BASE IMPEDANCE TENSOR:

PFEO EX: 0.9

PRED-EY:

0.915
MORIAALIZED TELLURIC TRAISSFER TEHSOR

$\begin{array}{rrrr}0.5409 & 0.1739 & 0.4245 & -0.3972 \\ -0.1810 & 0.2410 & 0.8270 & 0.3182\end{array}$

REMOTE TELLURIC TENSOR:

PRED-EX: 0.846 PRED-EY: 0.899

JEMEZ-EACA LOCATION (REMDTE)

JE-EQ-A

FEFIOD: 30

AYIS: 1

PHASE-COPEECTED

COHPEHSATED TELLURIC

ELLIPSE PARAMETERS

$\begin{array}{ll}\text { MAJOR AY1S: } & 0.936 \\ \text { MLHOR AXIS: } & 9.569\end{array}$

$\begin{array}{ll}\text { MLHOR AXIS: } & 9.569 \\ \text { AEIMUTH: } & 57.864\end{array}$

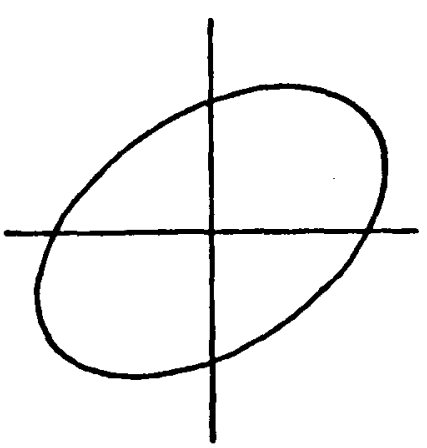




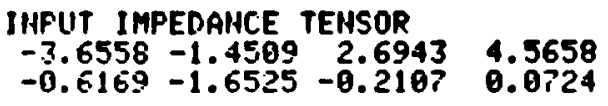

BASE IMFELANCE TENSOR:

FFE['EX: 0.9 PRED-EY: 0.915

JEMEZ-BACA LOCATION JE-E1B-A

FEFIOD: 30

AYIS: 1

PHASE-COPRECTED

COHFEHSATEO TELLURIC

ELLIPSE PARAIIETERS

MAJOR A\%IS:

MINOR GHIS:

AZIMUTH:

3.479

9.743

8.022

\section{(REMOTE)}

NORMALIZEO

2.8118
-0.8536

0.2248

URIC TRAN

NSFER TENSOR

REMOTE TELURIC TENSO:

PRED-EX: 6.827 PRED-EY: 0.718

$-0.8540$

0.4011

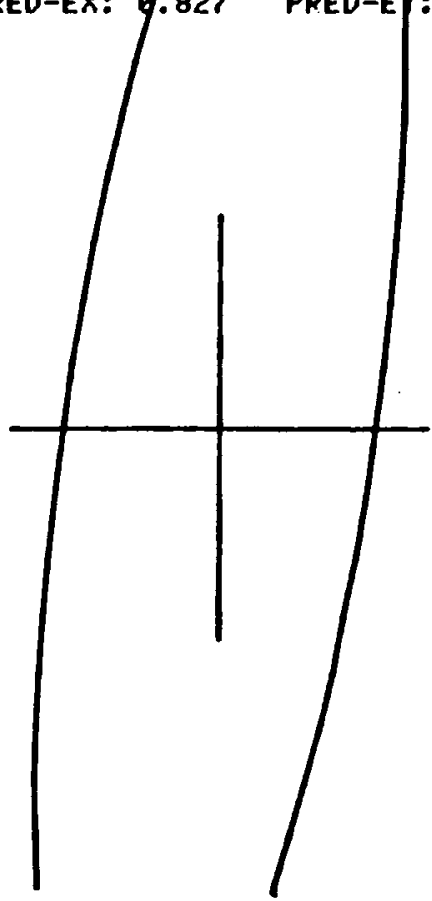

IHPUT IMPEDANCE TENSOR

$\begin{array}{llll}-0.3381 & -0.3118 & 1.4215 & 1.3354\end{array}$

$\begin{array}{llll}-0.4665 & -0.6179 & 0.0666 & 0.4649\end{array}$

RASE IMPEDANCE TENSOR:

PRE[-EX: 0.9 PRED-EY: 0.915
MORMALIZED TELLURIC TRAHSFER TEHSOR

$1.0677-0.0334 \quad 0.2517-0.0102$

$0.1826 \quad 0.1310 \quad 0.4200 \quad 0.0586$

REMOTE TELLURIC TENSOR:

PRED-EX: 0.966 PRED-EY: 0.684

JEMEZ-BACA LOCATIOH (REMOTE)

JE $-E 11-\hat{A}$

PEFIOT: 30

AKIS: 1

PHASE-CIRFECTED

COHPEHSGTED TELLURIC

ELLIFSE FARAMHETERS

MAJOP AKIS: 1.135

MIHOP AXIS: 0.355

HE!HUTH: 15.592

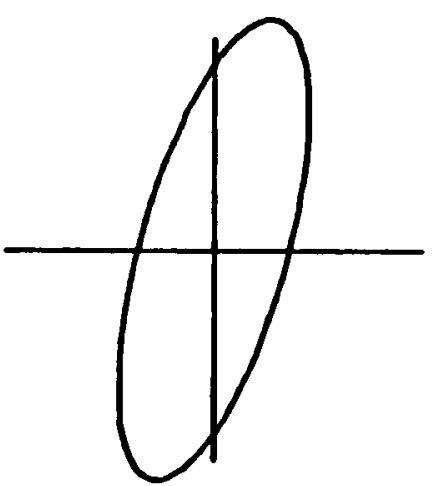


IHPUT IMPEOANCE TENSOR

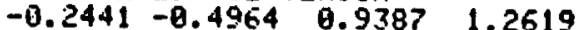

$-0.5906-1.1079-0.0358 \quad 0.3968$

RASE IMPEDANCE TENSOR:

PRED-EY: 0.9 PRED-EY: 0.915

JEMEZ-EACA LOCATIOH (REMOTE)

JE-12-A

FERIIIT: 30

A.:IS: 1

PHASE-COPRECTED

CÜMPEHSATED TELLURIC

ELLIFSE PARAMETERS

MAJJOR AYIS:

MIHUT AYIS:

AEIMUTH:

0.993

0.524

29.982
NORMALIZED TELLURIC TRAISFER TENSOR

$\begin{array}{llll}0.8523 & 0.1251 & 0.2868 & 0.0977\end{array}$

$0.14010 .16720 .6578 \quad 6.2003$

REMOTE TELLURIC TEHSOR:

PRED-EX: 0.884 PRE[-EY: 0.96

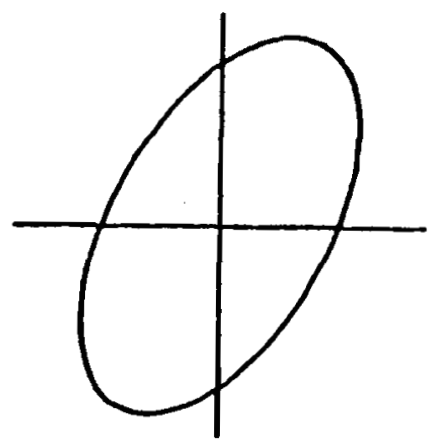

INPUT IMFEDANCE TEHSOR

$0.4931-0.11140 .85240 .1790$

$-0.2364-0.7890-0.5661$

B. 8006

BASE IMPEDAHCE TENSOR:

FFE[-EY: 0.9 PRED-EY: 0.915
NORMALIZED TELLURIC TRANSFER TENSOR

$0.3995-0.2608-0.1478 \quad 0.2341$

$\begin{array}{llll}-0.2190 & 0.2195 & 0.3971 & 0.2140\end{array}$

REMOTE TELLURIC TEHSOR:

PRED-E\%: 0.S2 PREL-EY: 0.86

JEMEZ-EACA LOCATIOH (REMOTE)

JE-E13-P

FERIOS: 30

Ax:15: 1

FHASE-CDRRECTED

COIAFENSATED TELLURIC

ELLIPSE FARAMETERS

MAJOF AKIS: 0.583

HIHOR AYIS: 0.216

AZIMUTH: $\quad-47.371$

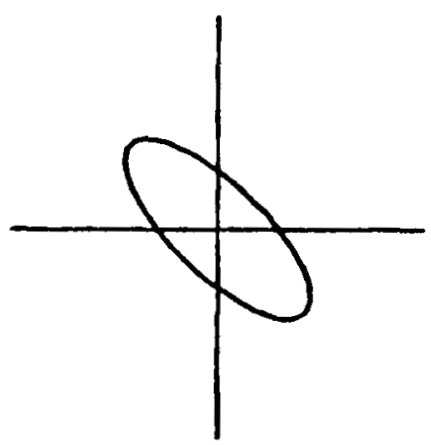


IHPUT IMPEDANCE TENSOR

$-0.1420 \quad 0.7999 \quad 0.5195$

$0.1726-0.5463-0.3630-0.3620$

RASE IMPECANCE TENSOR:

FFEC-EX: 0.9 PRED-EY: 0.915
HORMALIZED TELLURIC TRANSFER TENSOR

$0.5550 \quad 0.1526-0.2548-0.3648$

$\begin{array}{lllll}-0.2808 & 0.0004 & 0.1447 & 0.2784\end{array}$

REMOTE TELLURIC TENSOR:

PRED-EX: 0.554 PRED-EY: 0.256

JEMEZ-BACA LOCATION (RERTOTE)

JE-E 14-A

FEFIOR: 30

AMIS: 1

PHASE-CORRECTED

COMPENSATEO TELLURIC

ELLIFGE PAPAMETEFS

HAJOR AYYIS: 0.687

HIHUR AXIS: 0.013

AEIMUTH: -27.335

IHPUT IMPEDAHCE TENSOR

$0.1968 \quad 0.5414 \quad 0.5437$

$-0.2725 \quad 0.8451-6.3727$

0.0471

0.0600

EASE IMPEDANCE TENSOR:

FFED-EX: 0.9 FRED-EY: 0.915
MORMALIZED TELLURIC TRANSFER TENSOR

$0.2288-0.1923-0.2859-0.1334$

$\begin{array}{lllll}-0.1211 & 0.1676 & 0.0880 & -0.1239\end{array}$

REMOTE TELLURIC TEHSOR:

PRED-EX: 0.362 PRED-EY: 0.176

JEMEZ-EACA LOCATIOH (REMOTE)

$J E-E 15-A$

FEFIOS: 39

A:IS: 1

PHASE-CORRECTED

COHFEHSATED TELLURIC

ELLIFSE PARAMETEPS

MÂJIOP AYIS: 0.394

MINOP AKIS: 0.637

MZINUTH: -21.723

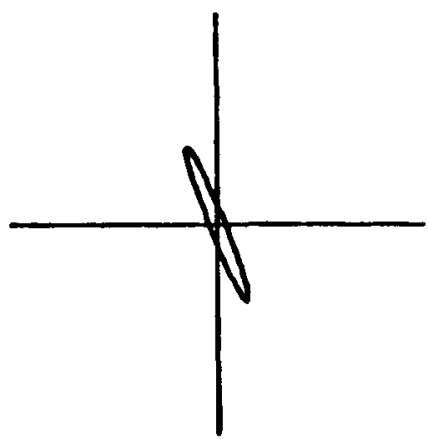


IHPUT IMPEDAHCE TEHSOR

$0.3458-0.4446 \quad 0.1680-0.1802$

$-0.4062-0.8633-0.4316-0.0984$

BASE IMPEOANCE TENSOR:

PFEL-E:: 0.9 PRED-EY: 0.915
MORMAL IZED TELLURIC TRANSFER TENSOR

$-0.0047-0.13490 .0383 \quad 0.3061$

$\begin{array}{lll}-0.2053 & 0.1291 & 0.4917\end{array}$

0.1770

PEMOTE TELLURIC TENSOR:

PRED-EK: 0.457 PRED-EY: 0.455

JEMEZ-BACA LOCATION (REMOTE)

JE-E 10-A

PERIOO: 39

AYIS: 1

PHÂEE-CDFRECTED

CDHFENSATED TELLURIC

ELLIFSE PAR:ANETERS

MAJOR ARIS: 0.534

HIHUR AKIS: 0.019

H2IMIJT: $\quad 86.912$

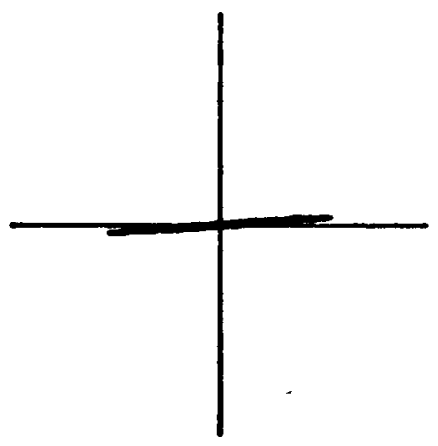

INPUT IMPEDANCE TENSOR

$0.1169-0.2747 \quad 0.4040$

MORMALIZED TELLURIC TRANSFER TEMSOR

$-0.5509-1.1913-0.6100$

0.0426

$0.2673-0.0457$

$0.0611 \quad 0.1517$

BASE IMPECHANCE TENSOR:

FRER-EY: 0.9 PRED-EY:

0.915

PEMOTE TELLURIC TENSOR:

PRED-EX: 0.547 PRED-EY: 0.689

JEMEZ-EACA LOCATIOH (REMOTE)

JE:-E $1>-\hat{\mathbf{A}}$

FERIOD: 30

AXIS: 1

FHASE-CORFECTED

COMFEHSATED TELLURIC

ELLIFGE FAPAMETERS

MáJür ayIS: 0.710

MIHIJR A.IS: 0.273

ALIMUTH: $\quad-87.6 E 7$

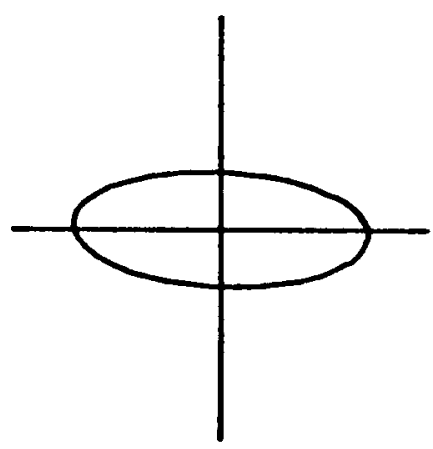


SECTION C

Mormalized and Compensated Telluric Ellipses (20 ohm-m) for Baca satellite 1 (B-1) determined at 50 sec. and 65 sec.

IHFUT IMPEDANCE TENSOR

- $9.9538-0.5754 \quad 1.0748$

$-0.4553-1.52350 .0331$

\subsection{8}

NOPMAL 12ED TELLURIC TRANSFER TENSOR

0.3331

IEHEZ-EACA (SATELLITE)

JE-ES1-1 (\$3É)

FEFIOI: 50

A.:15: 1

COMFEHSATED TELLURIC

ELLIFEE F'ARAHETEPS

COUNTER-CLOCKHISE POTATIOH

MAJOR HWIS: 2.179

MIHIOP HOIS: 1.109

HZIIIJTH:

13.699

CLOCKNISE ROTATION

MA.JOR A.IS: 1.684

MINIOR G.? 0.549

A2IIUTH: 37.007

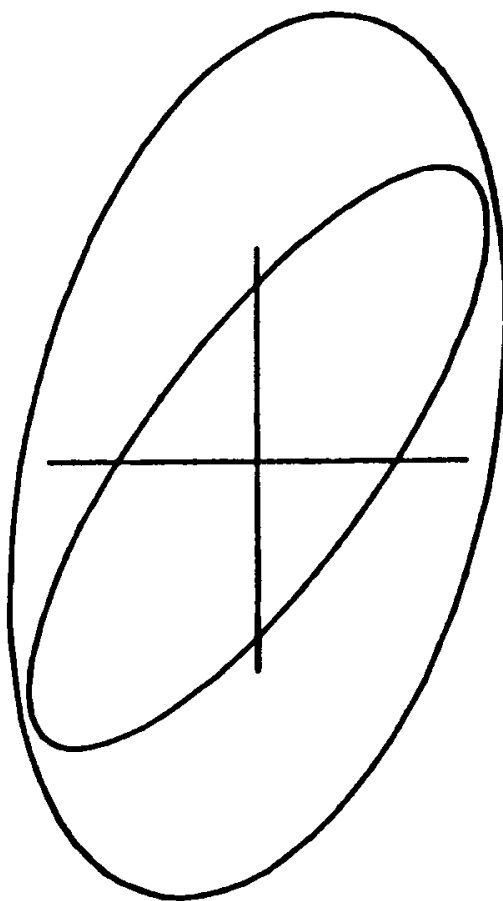

$-0.9538-0.5754$

$-8.4583-1.5235$

PRED-EX : 0.35 $\begin{array}{ll}1.5748 & 2.0988\end{array}$

0.03310 .3336

PRED-EY: 0.94
1.5414
0.1830

0.4674

0.1500

$0.7646-0.1892$

$0.9909 \quad 0.5326$

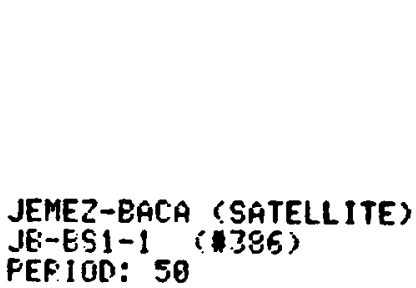

AXIS: 1

PHASE-CORRECTEO COHPENGATED TELLURIC

ELLIPSE FAPAHETERS

HAJOR AXIS: 1.847

HIHUR GYYS: 9.751

ADIMUTH: 23.455

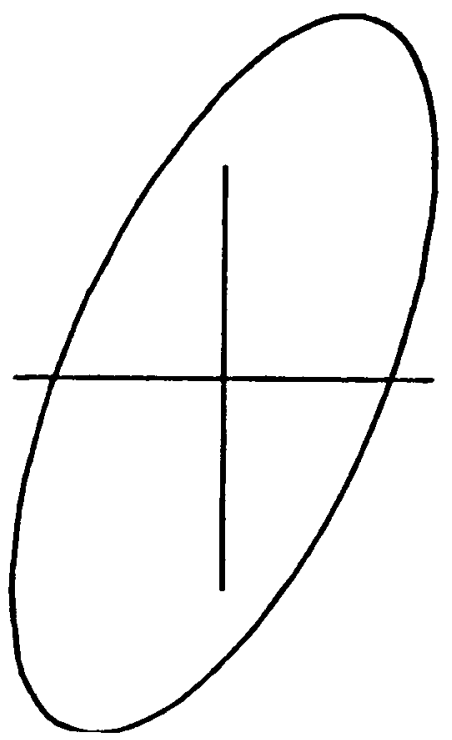


IHPUT IMPEOAHCE TEHSOR

$-0.9309-0.6908$ 1.1157

$-0.3285-1.4533-0.1826 \quad 9.4285$

1.9728
9.4285
0.9548

NORMALIZED TELLURIC TRANSFER TENSOR

$1.7607 \quad 0.488 \epsilon \quad 0.9183-0.1306$

$0.1402 \quad 0.3483 \quad 1.0158 \quad 0.6413$

FRED-E\%: 0.95585 PRED-EY: 0.9548

JEMEZ-BACA (SATELLITE)

JE-ESI-1 (\$8E)

AXIS: 1

COMPEISATEO TELLURIC

ELLIPSE FARAMETERS

COUNTER-CLOCKWISE ROTATIOH

MÂJOP AYIS: 2.411

MINOF AXIS: 1.363

MEIHUTH: $\quad 14.714$

CLOCKHISE ROTATIOH

MAJOF ARIS: 1.936
MIHOP A\%IS: 0.389

A2IHUTH: 30.113

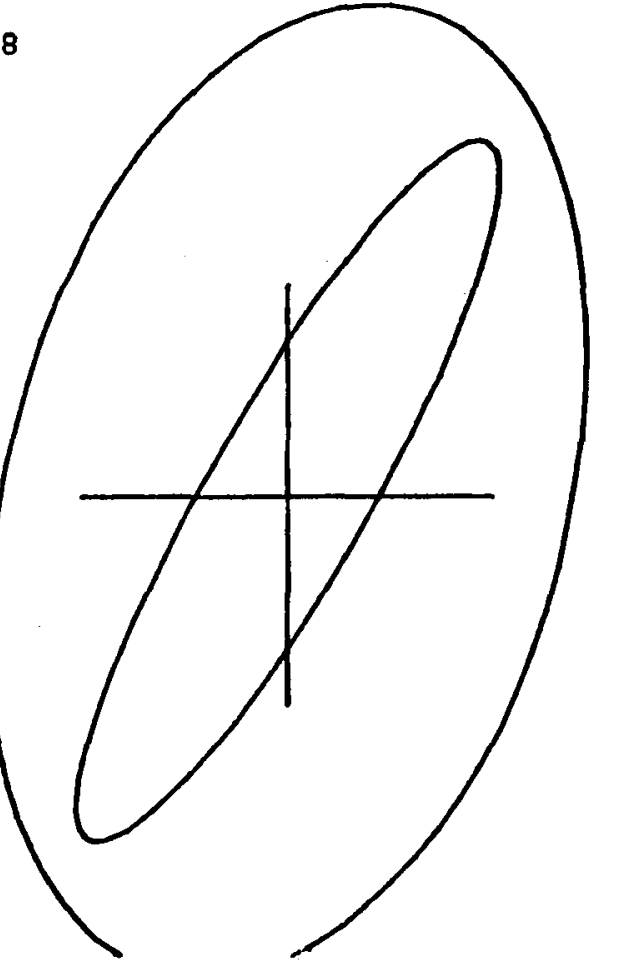

INPUT IMPEDANCE TENSOR

$\begin{array}{llll}-0.9367 & -0.2567 & 0.6455 & 1.5909 \\ -9.3010 & -1.1690 & -0.1931 & 0.3335\end{array}$

NORMALIZED TELLURIC TRANSFER TENSOR

$\begin{array}{llll}1.2750 & 0.5389 & 0.6234 & -6.3366 \\ 0.6801 & 0.3002 & 0.8374 & 0.4943\end{array}$

PRED-EK: 0.8715 PRED-EY: 0.83511

JEMEZ-BACA (SATELLITE)

JB-ES:1-1 (\$3B6?

PEFIOD: 65

Ax1S: 1

COMPENSATED TELLURIC

ELLIPSE PGRAMETERS

COUNTER-CLOCKRISE ROTATIOH

HAJUR AYIS: 2.623

AIHOF AHIS: 1.141

ALIMUTH: 14.044

CLOCKHISE ROTATIOH

MAJOR AYIS: 1.1E?

MIHOP AXIS: 0.393

GEIMUTH: $\quad 38.292$

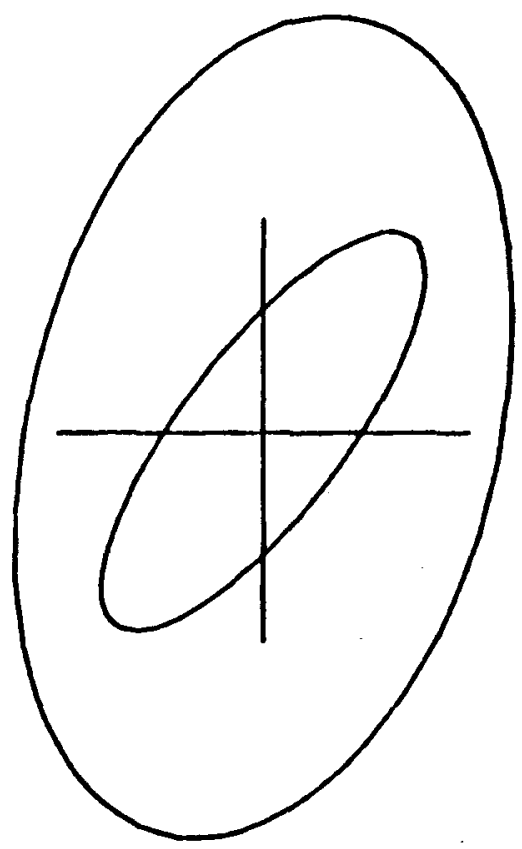


IHPUT IMPEDANHCE TENSOR

$-0.9200-0.6968 \quad 1.1157 \quad 1.9728$

$-6.3285-1.4533-0.1826$

0.4285
NORMALIZED TELLURIC TRANSFER TEMSOR

$\begin{array}{llll}1.7697 & 0.488 \epsilon & 0.9183 & -0.1306 \\ 0.1402 & 0.3483 & 1.0158 & 0.6413\end{array}$

JENEZ-EACG (SATELLITE)

$J E-E S 1-1$ ( $\$ B E)$

PERIOU: 65

A.XIS: 1

PHASE-CORRECTED

COMFENSGATED TELLUPIC

ELLIFSE PARAMETERS

MAJOR AYIS: 2.089

MIHÜR AXIS: 0.795

AZIHUTH:

19.604

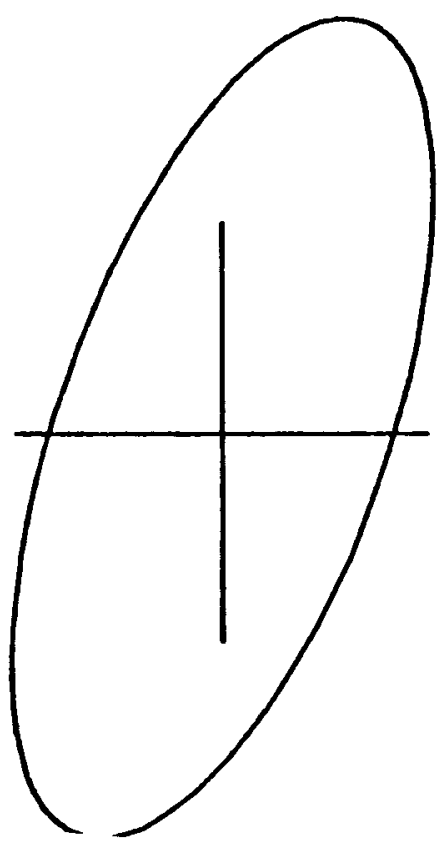

IHFUT IMPEDAHCE TEHSOR

$-0.8367-0.2567$
$-0.3019-1.1689-0.6455$

1. 5999

0.3335
NORMALIZED TELLURIC TRAHSFER TEMSOF

$\begin{array}{llll}1.2750 & 0.5389 & 0.6234 & -9.3306\end{array}$

$0.0801 \quad 0.3002 \quad 0.8374 \quad 0.4943$

JEMEZ-BACA (SATELLITE)

JE-ES1-1 (\$386)

AXIS: 1

FHASE-CORRECTED

COMFEHSATEL TELLURIC

ELLIFSE PARAMETERS

MAJOR AKIS: 1.505

MINOR AKIS: 0.676

A2INITH: $2 i .848$

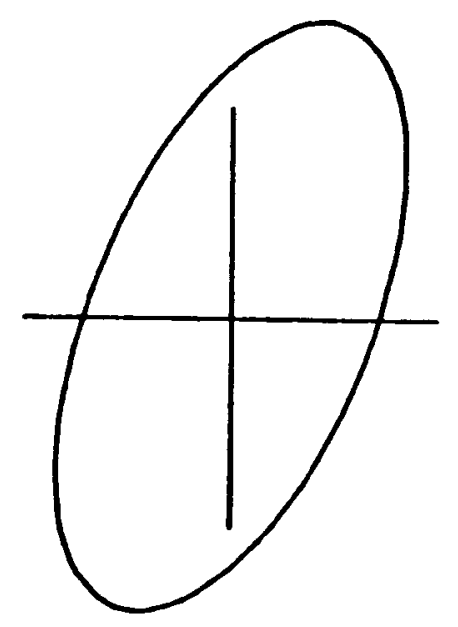


DATA SETS INCLUDED:

1345

$\$ 347$

379

$\$ 385$

$\$ 405$

$+416$

\begin{tabular}{|c|c|c|c|c|c|c|c|c|c|}
\hline FERIOD & HO & $\begin{array}{c}\text { PRED } \\
\text { EX }\end{array}$ & $\begin{array}{c}\text { PRED } \\
\text { EY }\end{array}$ & $\begin{array}{l}\text { ANGLLE } \\
\text { (CH) }\end{array}$ & RES-MAX & $\begin{array}{l}\text { PHASE } \\
\text { HAX }\end{array}$ & RES-MIN & $\begin{array}{l}\text { PHASE } \\
\text { HIH }\end{array}$ & SKEH \\
\hline $\begin{array}{r}5090 \\
3900 \\
5090 \\
1500 \\
1050 \\
750 \\
5.00 \\
590\end{array}$ & $\begin{array}{r}8 \\
12 \\
9 \\
10 \\
24 \\
26 \\
5 ? \\
5 ?\end{array}$ & $\begin{array}{l}0.95 \\
0.98 \\
0.98 \\
0.98 \\
0.95 \\
0.89 \\
0.84 \\
0.10\end{array}$ & $\begin{array}{l}0.96 \\
0.88 \\
0.90 \\
0.88 \\
0.88 \\
0.90 \\
0.86\end{array}$ & $\begin{array}{r}13.8 \\
8.4 \\
17.1 \\
15.4 \\
12.5 \\
19.1 \\
27.6\end{array}$ & $\begin{array}{l}14.97 \\
18.14 \\
18.39 \\
16.92 \\
14.22 \\
11.41 \\
10.47\end{array}$ & $\begin{array}{l}30.0 \\
37.6 \\
41.0 \\
43.9 \\
47.8 \\
39.6 \\
45.2\end{array}$ & $\begin{array}{l}2.99 \\
3.64 \\
2.92 \\
2.06 \\
2.09 \\
2.46 \\
2.00\end{array}$ & $\begin{array}{l}55.1 \\
39.1 \\
58.3 \\
61.5 \\
68.9 \\
58.3 \\
52.2\end{array}$ & $\begin{array}{l}0 . \\
0 . \\
0 . \\
0 . \\
0 . \\
0 . \\
0 .\end{array}$ \\
\hline
\end{tabular}

IHFEGAHCE TEHSOR

\begin{tabular}{|c|c|c|c|c|c|c|c|}
\hline EF:100 & $E(2 \times 3)$ & $I H(2 \times \%)$ & RE (ZXY) & $\operatorname{IH}(Z X Y) \operatorname{RE}(Z Y X)$ & $\operatorname{IH}(2 Y X)$ & $E(Z Y Y)$ & IM(ZYY) \\
\hline $\begin{array}{l}5960 \\
39690 \\
2960 \\
1590 \\
1950 \\
750 \\
500\end{array}$ & $\begin{array}{l}-0.0187 \\
-0.0025 \\
-0.0449 \\
-0.0617 \\
-0.0615 \\
-0.0851 \\
-0.0919\end{array}$ & $\begin{array}{l}-0.0144 \\
-0.0241 \\
-0.0541 \\
-0.0365 \\
-0.0085 \\
-0.0520 \\
-0.1191\end{array}$ & $\begin{array}{l}0.1028 \\
0.1395 \\
0.1579 \\
0.1618 \\
0.1691 \\
0.1984 \\
0.2183\end{array}$ & 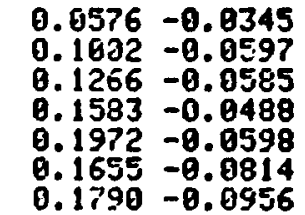 & $\begin{array}{l}-0.0483 \\
-0.0537 \\
-0.0798 \\
-0.0792 \\
-0.0896 \\
-0.1193 \\
-0.1624\end{array}$ & $\begin{array}{r}0.0118 \\
0.0041 \\
-0.0803 \\
0.0058 \\
0.0104 \\
0.0013 \\
-0.0054\end{array}$ & \\
\hline
\end{tabular}



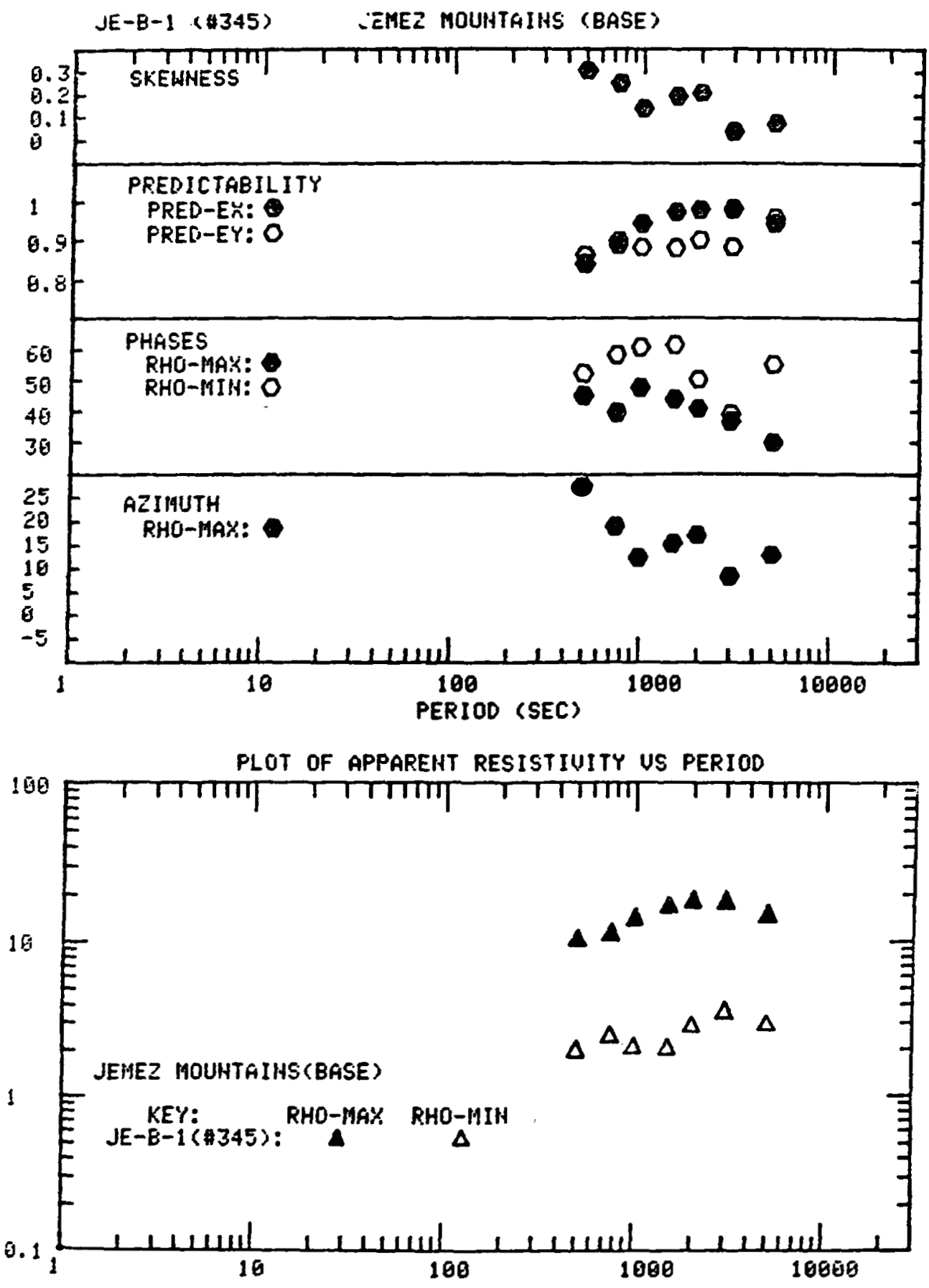

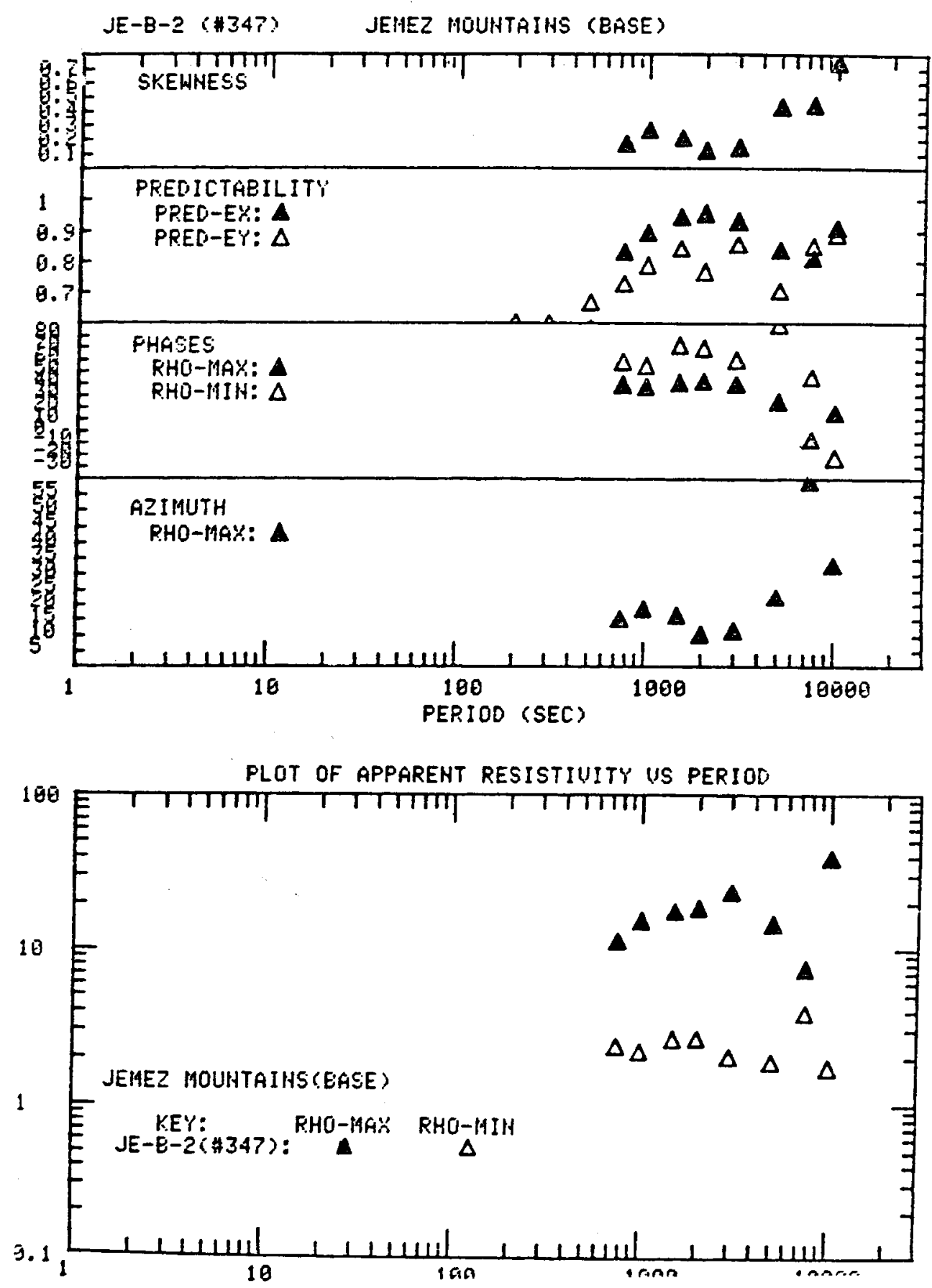


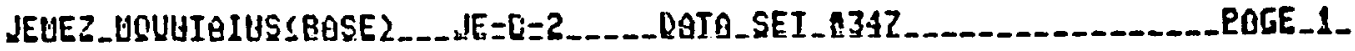

\begin{tabular}{|c|c|c|c|c|c|c|c|c|c|}
\hline PER 1OD & HU & $\begin{array}{l}\text { FRED } \\
\text { E\% }\end{array}$ & $\begin{array}{l}\text { PRED } \\
\text { EY }\end{array}$ & $\begin{array}{l}\text { AHGLE } \\
\text { (CH) }\end{array}$ & RES-MAX & $\underset{\text { MAX }}{\text { FMASE }}$ & RES-MIH & $\begin{array}{c}\text { PHASE } \\
\text { HIII }\end{array}$ & SKEW \\
\hline $\begin{array}{r}10900 \\
7500 \\
5000 \\
3009 \\
3009 \\
1500 \\
1000 \\
750 \\
500 \\
300 \\
200\end{array}$ & $\begin{array}{r}4 \\
5 \\
7 \\
12 \\
18 \\
11 \\
12 \\
17 \\
30 \\
53 \\
90\end{array}$ & $\begin{array}{l}0.91 \\
0.91 \\
0.84 \\
0.93 \\
0.95 \\
0.95 \\
0.89 \\
0.83 \\
0.58 \\
0.45 \\
0.54\end{array}$ & $\begin{array}{l}0.89 \\
0.85 \\
0.71 \\
0.86 \\
0.77 \\
0.84 \\
0.79 \\
0.73 \\
0.67 \\
0.66 \\
0.60\end{array}$ & $\begin{array}{l}33.0 \\
59.1 \\
22.8 \\
11.6 \\
10.5 \\
16.5 \\
18.7 \\
15.3\end{array}$ & $\begin{array}{r}39.41 \\
7.61 \\
14.83 \\
23.93 \\
19.73 \\
17.71 \\
15.48 \\
11.45\end{array}$ & $\begin{array}{l}15.4 \\
-7.3 \\
24.7 \\
39.4 \\
41.7 \\
40.6 \\
37.0 \\
33.1\end{array}$ & $\begin{array}{l}1.77 \\
3.95 \\
1.92 \\
2.05 \\
2.69 \\
2.67 \\
2.22 \\
2.39\end{array}$ & $\begin{array}{r}-22.9 \\
44.9 \\
89.1 \\
59.7 \\
69.8 \\
71.9 \\
55.0 \\
58.2\end{array}$ & $\begin{array}{l}0.75 \\
0.45 \\
0.43 \\
0.15 \\
0.13 \\
0.21 \\
0.26 \\
0.17\end{array}$ \\
\hline
\end{tabular}

IMFEUAHCE TENSOR

\begin{tabular}{|c|c|c|c|c|c|c|c|c|}
\hline 8100 & & $(2 \times ! 9)$ & $R E(Z X Y i)$ & $\operatorname{In}(2 X Y)$ & RE $(Z Y \%)$ & ; & RE & $1 H(Z Y Y)$ \\
\hline $\begin{array}{l}19500 \\
7500 \\
5900 \\
3000 \\
2000 \\
1500 \\
1900 \\
750\end{array}$ & $\begin{array}{l}-0.0840 \\
-0.0412 \\
-0.962 . \\
-0.9356 \\
-0.0455 \\
-0.9721 \\
-0.9796 \\
-0.0676\end{array}$ & $\begin{array}{r}-0.0749 \\
9.0052 \\
-0.0294 \\
-0.0271 \\
-0.0614 \\
-0.0346 \\
-9.0717 \\
-9.0312\end{array}$ & $\begin{array}{l}0.106 \\
0.653 \\
0.694 \\
0.143 \\
0.155 \\
0.173 \\
0.219 \\
0.205\end{array}$ & $\begin{array}{l}0.630 \\
8.843 \\
0.125 \\
0.144 \\
0.143 \\
0.118 \\
0.166\end{array}$ & & $\begin{array}{r}-0.0099 \\
0.0932 \\
-0.9509 \\
-0.0516 \\
-0.0766 \\
-0.1009 \\
-0.1048 \\
-0.1146\end{array}$ & $\begin{array}{r}0.0117 \\
-0.0024 \\
0.0152 \\
0.0195 \\
0.0091 \\
0.0957 \\
0.0057 \\
0.0030\end{array}$ & $\begin{array}{r}-0.0244 \\
-0.0283 \\
-0.0126 \\
-0.0063 \\
0.0093 \\
0.0173 \\
0.0039 \\
0.0126\end{array}$ \\
\hline
\end{tabular}

\begin{tabular}{|c|c|c|c|c|c|c|c|c|c|}
\hline PERIO0 & NU & $\begin{array}{l}\text { PRED } \\
\text { E\% }\end{array}$ & $\begin{array}{c}\text { PREO } \\
\text { EY }\end{array}$ & $\begin{array}{l}\text { ANGLEE } \\
\text { (CH) }\end{array}$ & RES-MAX & $\underset{\text { MAK }}{\text { PHASE }}$ & RES-MIN & $\begin{array}{l}\text { PHASE } \\
\text { MIH }\end{array}$ & SK.EH \\
\hline $\begin{array}{r}5000 \\
3090 \\
2090 \\
1550 \\
1099 \\
750 \\
500 \\
350 \\
250 \\
159 \\
199 \\
75\end{array}$ & $\begin{array}{r}8 \\
13 \\
8 \\
20 \\
25 \\
27 \\
28 \\
79 \\
109 \\
130 \\
199 \\
323 \\
408\end{array}$ & $\begin{array}{l}0.82 \\
0.82 \\
0.73 \\
0.83 \\
0.87 \\
0.87 \\
0.82 \\
0.85 \\
0.84 \\
0.84 \\
0.82 \\
0.79\end{array}$ & $\begin{array}{l}0.90 \\
0.93 \\
0.85 \\
0.95 \\
0.93 \\
0.95 \\
0.92 \\
0.92 \\
6.92 \\
0.91 \\
0.90 \\
0.88 \\
0.70\end{array}$ & $\begin{array}{l}33.6 \\
68.6 \\
6.4 \\
26.3 \\
36.1 \\
51.3 \\
47.9 \\
36.4 \\
40.7 \\
50.9 \\
53.8 \\
55.2\end{array}$ & $\begin{array}{r}15.81 \\
9.59 \\
8.20 \\
11.81 \\
13.09 \\
9.17 \\
9.39 \\
13.08 \\
13.17 \\
11.16 \\
10.57 \\
9.74\end{array}$ & $\begin{array}{l}49.7 \\
47.9 \\
24.2 \\
23.7 \\
47.4 \\
51.3 \\
51.2 \\
50.4 \\
57.2 \\
55.4 \\
57.3 \\
58.3\end{array}$ & $\begin{array}{l}0.26 \\
6.48 \\
3.87 \\
2.51 \\
1.14 \\
0.42 \\
1.18 \\
2.99 \\
2.59 \\
2.08 \\
0.99 \\
0.90\end{array}$ & $\begin{array}{r}81.8 \\
-13.4 \\
63.4 \\
60.6 \\
55.1 \\
79.9 \\
62.6 \\
57.5 \\
73.8 \\
83.4 \\
73.4 \\
72.5\end{array}$ & $\begin{array}{l}0.34 \\
0.61 \\
0.43 \\
8.46 \\
0.40 \\
0.65 \\
8.47 \\
0.33 \\
0.27 \\
0.40 \\
0.45 \\
0.45\end{array}$ \\
\hline
\end{tabular}


IMPEDANCE TENSOR.

\begin{tabular}{|c|c|c|c|c|c|c|c|c|}
\hline ER I0! & $R E(Z X Y)$ & $\operatorname{IH}(Z X X)$ & $R E(Z X Y)$ & $I M(Z X Y)$ & RE (ZYK) & $I M(Z Y X)$ & RE (ZYY) & $\operatorname{IM}(Z Y Y)$ \\
\hline $\begin{array}{l}5909 \\
3000 \\
2009 \\
1500 \\
1909 \\
750 \\
590 \\
395 \\
200 \\
159 \\
190 \\
75\end{array}$ & $\begin{array}{l}-0.0628 \\
-0.0327 \\
-0.0398 \\
-0.1074 \\
-0.1222 \\
-0.1591 \\
-0.1484 \\
-0.1750 \\
-0.2219 \\
-0.3966 \\
-0.3483 \\
-0.3944\end{array}$ & $\begin{array}{l}-0.0216 \\
-0.1047 \\
-0.0703 \\
-0.0598 \\
-0.0850 \\
-0.1077 \\
-0.1295 \\
-0.1478 \\
-0.1638 \\
-0.1957 \\
-0.2899 \\
-0.2956\end{array}$ & $\begin{array}{l}0.0449 \\
0.1288 \\
0.1274 \\
0.1549 \\
0.1361 \\
0.0729 \\
0.1345 \\
0.2508 \\
0.1963 \\
0.1331 \\
0.1957 \\
0.2228\end{array}$ & $\begin{array}{r}9.0836 \\
-0.0044 \\
0.0525 \\
0.0608 \\
0.1363 \\
0.1090 \\
0.1400 \\
0.2816 \\
0.3940 \\
0.3876 \\
0.3375 \\
0.3530\end{array}$ & $\begin{array}{l}-0.0388 \\
-0.0571 \\
-0.0473 \\
-0.0717 \\
-0.0883 \\
-0.0910 \\
-0.1076 \\
-0.1651 \\
-0.1856 \\
-0.2441 \\
-0.2603 \\
-0.2736\end{array}$ & $\begin{array}{l}-0.0284 \\
-0.0740 \\
-0.0941 \\
-0.0986 \\
-0.1139 \\
-0.1453 \\
-0.1952 \\
-0.2637 \\
-0.3325 \\
-0.3759 \\
-0.4877 \\
-0.5662\end{array}$ & $\begin{array}{r}0 . \\
0 . \\
-8 . \\
0 . \\
-0 . \\
-0 . \\
-0 . \\
-0 . \\
0 . \\
-0 . \\
-0 . \\
-0 .\end{array}$ & $\begin{array}{l}0 \\
0.0 \\
0.0 \\
0\end{array}$ \\
\hline
\end{tabular}
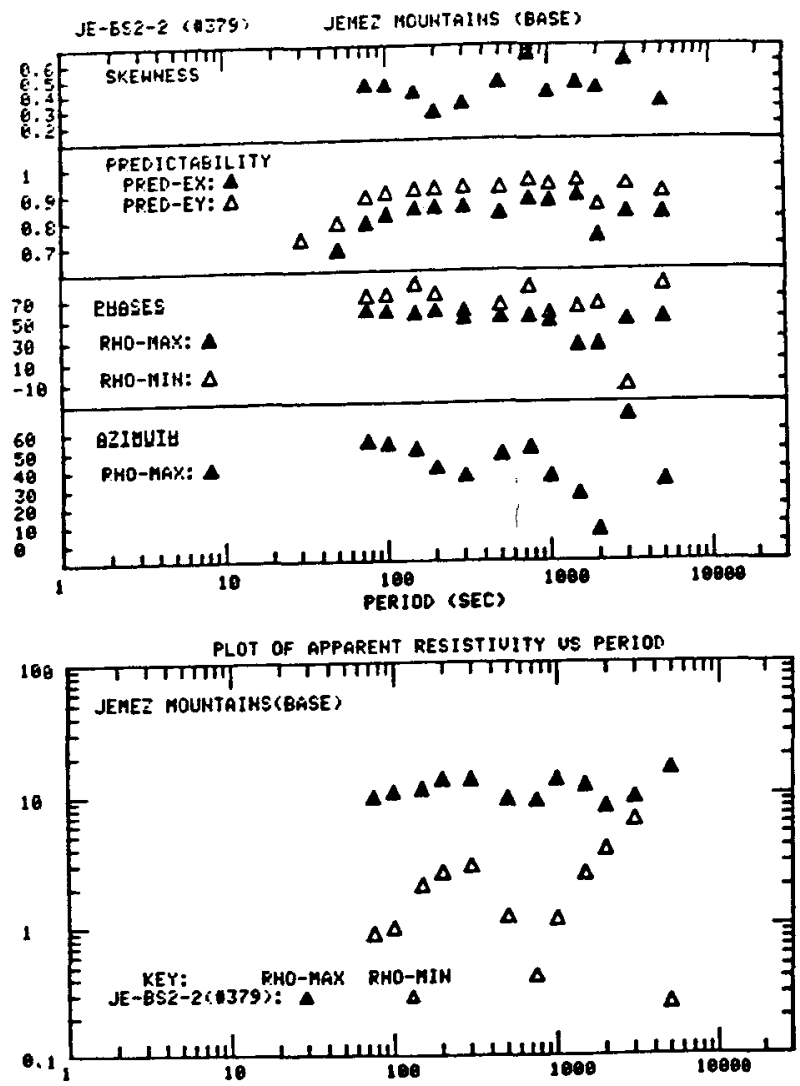


\begin{tabular}{|c|c|c|c|c|c|c|c|c|c|}
\hline PERIOD & HU & $\begin{array}{l}\text { PRED } \\
\text { Ex }\end{array}$ & $\begin{array}{l}\text { PRED } \\
\text { EY }\end{array}$ & $\begin{array}{l}\text { ANGLE } \\
\text { (CH) }\end{array}$ & RES-MAX. & $\begin{array}{l}\text { PHASE } \\
\text { HAX }\end{array}$ & RES-MIN & $\begin{array}{c}\text { PHASE } \\
\text { MIH }\end{array}$ & SKEW \\
\hline $\begin{array}{l}5090 \\
30019 \\
2000 \\
1500 \\
1900 \\
750 \\
500 \\
301 \\
200\end{array}$ & $\begin{array}{l}11 \\
17 \\
12 \\
13 \\
25 \\
36 \\
41 \\
89 \\
165\end{array}$ & $\begin{array}{l}0.99 \\
0.99 \\
0.99 \\
0.98 \\
0.94 \\
0.90 \\
0.99 \\
0.81 \\
0.68\end{array}$ & $\begin{array}{l}0.96 \\
0.98 \\
0.96 \\
0.96 \\
0.92 \\
0.87 \\
0.82 \\
0.79 \\
0.73\end{array}$ & $\begin{array}{l}14.4 \\
14.2 \\
16.4 \\
18.9 \\
16.8 \\
14.8 \\
12.8 \\
20.5\end{array}$ & $\begin{array}{l}25.79 \\
30.69 \\
27.13 \\
26.82 \\
18.58 \\
14.54 \\
13.84 \\
12.92\end{array}$ & $\begin{array}{l}45.9 \\
38.8 \\
39.0 \\
38.4 \\
36.7 \\
46.1 \\
45.2 \\
58.8\end{array}$ & $\begin{array}{l}1.94 \\
2.62 \\
2.46 \\
2.53 \\
2.88 \\
3.11 \\
2.96 \\
3.37\end{array}$ & $\begin{array}{l}65.9 \\
61.7 \\
59.2 \\
58.1 \\
51.1 \\
48.2 \\
54.8 \\
55.2\end{array}$ & $\begin{array}{l}0.25 \\
0.18 \\
0.22 \\
0.28 \\
0.31 \\
0.19 \\
0.12 \\
0.21\end{array}$ \\
\hline $\begin{array}{r}150 \\
108 \\
75 \\
56 \\
30 \\
20\end{array}$ & $\begin{array}{r}171 \\
371 \\
598 \\
65.3 \\
940 \\
1600\end{array}$ & $\begin{array}{l}0.66 \\
0.71 \\
0.86 \\
0.87 \\
0.60 \\
0.30\end{array}$ & $\begin{array}{l}0.71 \\
0.81 \\
0.89 \\
0.91 \\
0.79 \\
0.34\end{array}$ & $\begin{array}{l}38.8 \\
32.5 \\
26.7\end{array}$ & $\begin{array}{l}14.86 \\
22.82 \\
26.60\end{array}$ & $\begin{array}{l}49.7 \\
51.1 \\
48.7\end{array}$ & $\begin{array}{l}3.19 \\
6.44 \\
7.57\end{array}$ & $\begin{array}{l}74.7 \\
79.2 \\
73.6\end{array}$ & $\begin{array}{l}0.42 \\
0.38 \\
0.29\end{array}$ \\
\hline
\end{tabular}

IMPEDANCE TENSOR

\begin{tabular}{|c|c|c|c|c|c|c|c|c|}
\hline PERIDD & RE (ZKX) & $\operatorname{IM}(2 X X)$ & $\operatorname{RE}(Z X Y)$ & $\operatorname{IM}(Z X Y)$ & RE (ZYY) & $I M(Z Y X)$ & $\operatorname{RE}(Z Y Y)$ & IM(ZYY) \\
\hline $\begin{array}{l}5000 \\
3009 \\
2000 \\
1509 \\
1990 \\
759 \\
500 \\
390 \\
199 \\
75 \\
50\end{array}$ & $\begin{array}{l}-0.0419 \\
-0.056 .4 \\
-0.0780 \\
-0.9794 \\
-0.0799 \\
-0.0716 \\
-0.0613 \\
-0.1133 \\
-0.4509 \\
-0.6632 \\
-0.7952\end{array}$ & $\begin{array}{l}-0.0337 \\
-0.0330 \\
-0.0405 \\
-0.0892 \\
-0.0905 \\
-0.0467 \\
-0.0464 \\
-0.6961 \\
-0.2573 \\
-0.1229 \\
-0.1380\end{array}$ & $\begin{array}{l}0.1070 \\
0.1674 \\
0.1885 \\
0.2179 \\
0.2361 \\
0.2282 \\
0.2544 \\
0.2733 \\
0.3744 \\
0.5368 \\
0.8763\end{array}$ & $\begin{array}{l}0.1093 \\
0.1369 \\
0.1576 \\
0.1612 \\
0.1636 \\
0.1956 \\
0.2575 \\
0.3398 \\
0.5686 \\
0.9694 \\
1.2131\end{array}$ & $\begin{array}{l}-0.0227 \\
-0.0402 \\
-0.0540 \\
-0.0614 \\
-0.0836 \\
-0.1059 \\
-0.1071 \\
-0.1559 \\
-0.2881 \\
-0.3602 \\
-0.4446\end{array}$ & $\begin{array}{l}-0.0463 \\
-0.0632 \\
-0.0738 \\
-0.0996 \\
-0.1122 \\
-0.1124 \\
-0.1468 \\
-0.2145 \\
-0.4825 \\
-0.6342 \\
-0.8479\end{array}$ & $\begin{array}{r}-0.0087 \\
0.0128 \\
0.0124 \\
0.0164 \\
-0.0042 \\
0.0068 \\
0.0083 \\
-0.0088 \\
-0.0968 \\
-0.0313 \\
0.0086\end{array}$ & $\begin{array}{r}0.0977 \\
0.0064 \\
0.0078 \\
0.0027 \\
-0.0674 \\
-0.0945 \\
0.0078 \\
0.0118 \\
0.0040 \\
0.0698 \\
0.0780\end{array}$ \\
\hline
\end{tabular}



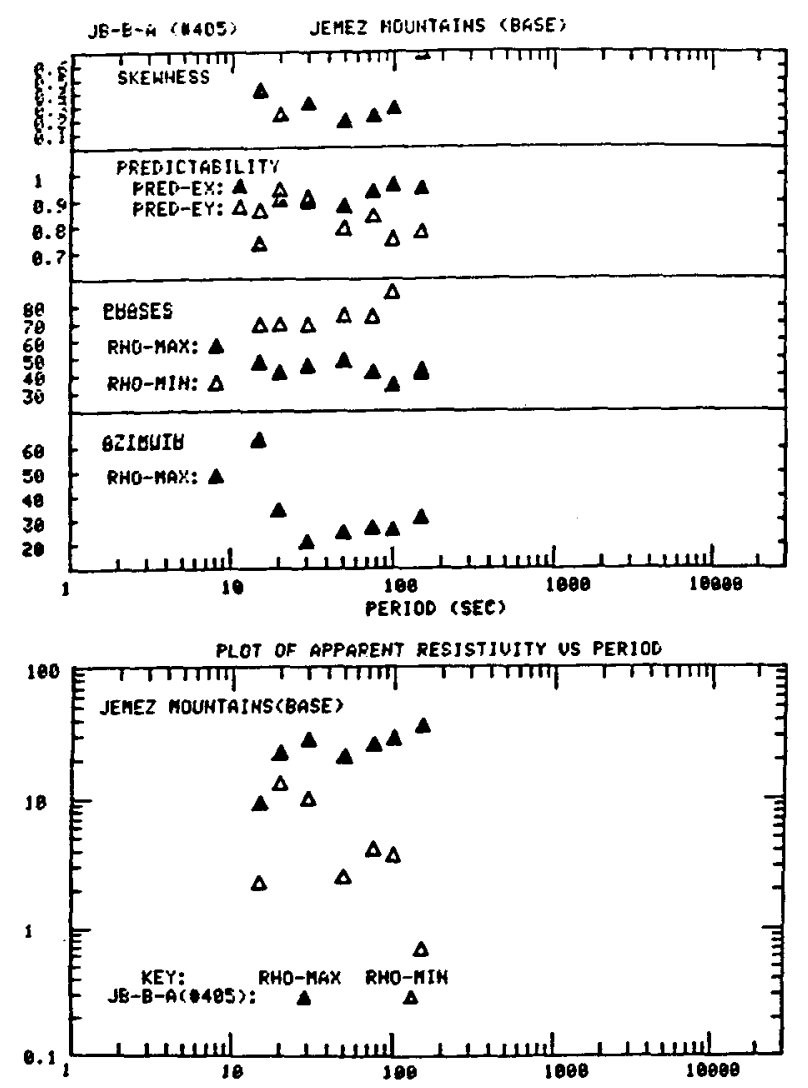

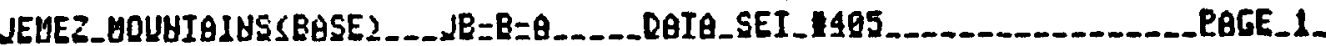

\begin{tabular}{|c|c|c|c|c|c|c|c|c|c|}
\hline$=R 100$ & $\mathrm{NU}$ & $\begin{array}{c}\text { PREDO } \\
\text { EXX }\end{array}$ & $\begin{array}{c}\text { PRED } \\
\text { EY }\end{array}$ & $\begin{array}{l}\text { ANGLE } \\
\text { (CH) }\end{array}$ & RES-MAK & $\begin{array}{c}\text { PHASE } \\
\text { MAX. }\end{array}$ & RES-MIH & $\begin{array}{c}\text { PHASE } \\
\text { IIIH }\end{array}$ & SKEH \\
\hline $\begin{array}{l}150 \\
198 \\
75 \\
54 \\
30 \\
20 \\
15\end{array}$ & $\begin{array}{r}11 \\
15 \\
21 \\
32 \\
81 \\
199 \\
140\end{array}$ & $\begin{array}{l}0.95 \\
0.96 \\
0.94 \\
0.88 \\
0.98 \\
0.91 \\
0.74\end{array}$ & $\begin{array}{l}0.78 \\
0.76 \\
0.84 \\
0.80 \\
0.92 \\
0.94 \\
0.86\end{array}$ & $\begin{array}{l}31.4 \\
26.1 \\
27.0 \\
25.2 \\
21.2 \\
34.5 \\
63.5\end{array}$ & $\begin{array}{r}36.10 \\
29.17 \\
26.00 \\
21.36 \\
28.53 \\
22.66 \\
9.46\end{array}$ & $\begin{array}{l}43.4 \\
34.8 \\
42.2 \\
48.7 \\
45.7 \\
41.8 \\
47.8\end{array}$ & $\begin{array}{r}0.70 \\
3.76 \\
4.16 \\
2.57 \\
10.15 \\
13.21 \\
2.33\end{array}$ & $\begin{array}{l}41.4 \\
88.2 \\
74.3 \\
74.7 \\
69.3 \\
69.5 \\
69.3\end{array}$ & $\begin{array}{l}\text { B. } \\
0 \\
0\end{array}$ \\
\hline
\end{tabular}

IMPEGAHCE TENSTR

\begin{tabular}{|c|c|c|c|c|c|c|c|c|}
\hline EPIOD & RE $(Z Y X)$ & $\operatorname{In}(Z X X)$ & PE (ZXY) & $\operatorname{IM}(Z X Y)$ & RE (ZYX) & $\operatorname{IM}(Z Y X)$ & RE (ZYY) & $I M(Z Y Y)$ \\
\hline $\begin{array}{r}150 \\
130 \\
75 \\
50 \\
30 \\
20 \\
15\end{array}$ & $\begin{array}{l}-0.7213 \\
-0.6110 \\
-0.5180 \\
-0.4322 \\
-0.9212 \\
-0.9718 \\
-0.9907\end{array}$ & $\begin{array}{r}-0.3269 \\
-0.0864 \\
-9.2503 \\
-0.3976 \\
9.0834 \\
9.2768 \\
0.2893\end{array}$ & $\begin{array}{l}0.6440 \\
0.7877 \\
9.7980 \\
0.8399 \\
1.36 \epsilon 1 \\
1.4920 \\
0.6113\end{array}$ & $\begin{array}{l}0.5434 \\
0.6978 \\
0.8192 \\
0.9507 \\
1.5735 \\
1.5531 \\
0.6970\end{array}$ & $\begin{array}{l}-0.2682 \\
-0.2175 \\
-0.3205 \\
-0.2578 \\
-0.6180 \\
-1.0666 \\
-0.8929\end{array}$ & $\begin{array}{l}-0.3169 \\
-0.4254 \\
-0.5716 \\
-0.6362 \\
-1.2032 \\
-1.7364 \\
-1.4421\end{array}$ & $\begin{array}{r}-0.1469 \\
0.1838 \\
0.1648 \\
0.1640 \\
-0.1644 \\
0.0968 \\
-0.0142\end{array}$ & $\begin{array}{l}0.2870 \\
0.0269 \\
0.0354 \\
0.1334 \\
0.1870 \\
0.2235 \\
0.3415\end{array}$ \\
\hline
\end{tabular}



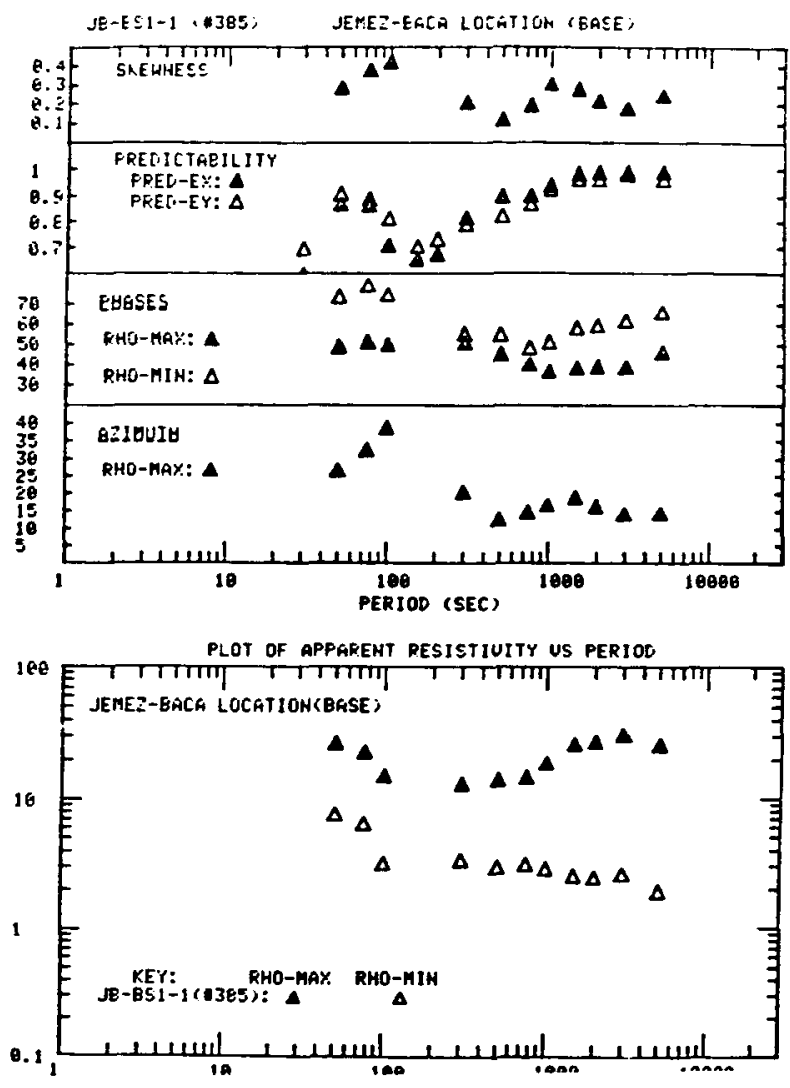

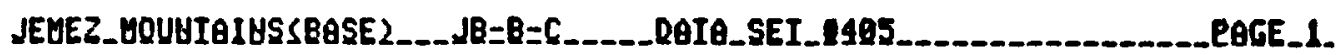

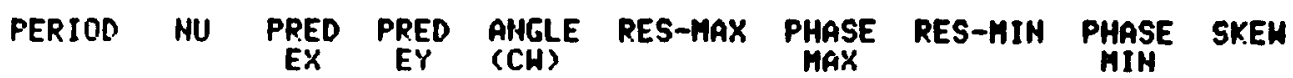

\begin{tabular}{|c|c|c|c|c|c|c|c|}
\hline $\begin{array}{r}11 \\
17 \\
29 \\
43 \\
93 \\
129\end{array}$ & $\begin{array}{l}0.52 \\
0.78 \\
0.98 \\
0.89 \\
0.83 \\
0.85\end{array}$ & $\begin{array}{l}0.72 \\
0.79 \\
0.88 \\
0.74 \\
0.78 \\
0.87\end{array}$ & $\begin{array}{l}23.7 \\
12.8 \\
27.3 \\
24.9\end{array}$ & $\begin{array}{l}23.48 \\
19.49 \\
20.46 \\
23.75\end{array}$ & $\begin{array}{l}47.1 \\
48.3 \\
36.5 \\
31.3\end{array}$ & $\begin{array}{l}3.24 \\
2.24 \\
6.36 \\
8.15\end{array}$ & $\begin{array}{l}75.9 \\
64.4 \\
73.9 \\
60.5\end{array}$ \\
\hline
\end{tabular}

$15 \quad 147 \quad 0.68 \quad 0.75$

$\begin{array}{rrrr}10 & 181 & 0.15 & 0.25 \\ 8 & 275 & 0.07 & 0.07\end{array}$

IIAPEDANCE TEHSOR.

$\begin{array}{rllllllll}\text { PERIOO } & \text { RE(ZXX) IM(ZXX) RE(ZXY) IM(ZXY) RE(ZYX) IM(ZYX) RE(ZYY) IM(ZYY) } \\ 75 & -0.4763 & -0.1194 & 0.6970 & 0.8974 & -0.2671 & -0.4781 & 0.1307 & 0.1383 \\ 59 & -0.2657 & -0.0338 & 0.8757 & 1.0329 & -0.2569 & -0.4371 & 0.1175 & 0.1478 \\ 39 & -0.8824 & 0.1285 & 1.2235 & 1.1709 & -0.5458 & -0.9179 & 0.1054 & 0.9832 \\ 29 & -0.9610 & 0.2321 & 1.8403 & 1.1948 & -0.9469 & -1.3115 & 0.0980 & 0.3604\end{array}$



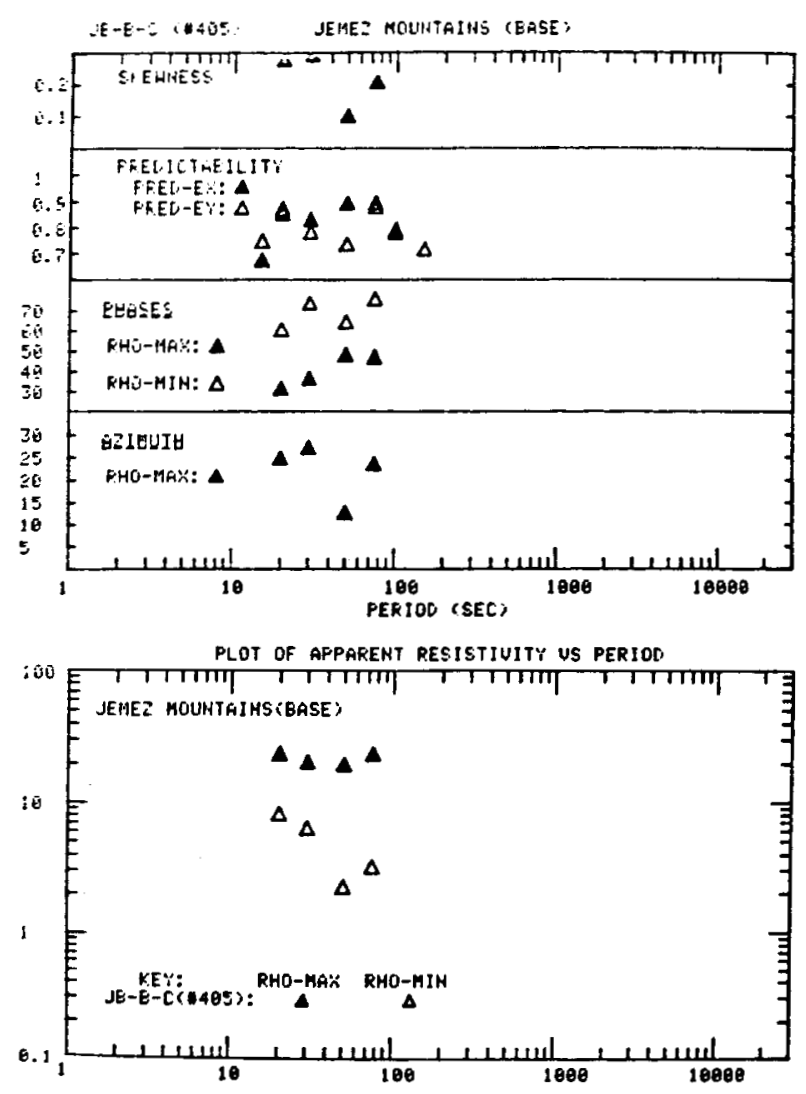

\begin{tabular}{|c|c|c|c|c|c|c|c|c|c|}
\hline PEFIOD & HU & $\begin{array}{l}\text { FRED } \\
\text { EX. }\end{array}$ & $\begin{array}{c}\text { PRED } \\
\text { EY }\end{array}$ & $\begin{array}{l}\text { AHGLE } \\
\text { (CH) }\end{array}$ & RES-MAX & $\begin{array}{l}\text { PHASE } \\
\text { MAX }\end{array}$ & RES-HIN & $\begin{array}{l}\text { PHASE } \\
\text { MIII }\end{array}$ & SKEH \\
\hline $\begin{array}{r}5000 \\
5050 \\
2090 \\
1590 \\
1909 \\
755 \\
5190 \\
350 \\
200 \\
150 \\
100 \\
75 \\
50 \\
30 \\
20\end{array}$ & $\begin{array}{r}10 \\
19 \\
122 \\
13 \\
19 \\
38 \\
58 \\
64 \\
95 \\
187 \\
312 \\
453 \\
571 \\
1155 \\
1993\end{array}$ & $\begin{array}{l}0.77 \\
0.86 \\
9.89 \\
0.72 \\
0.68 \\
0.69 \\
9.68 \\
0.64 \\
9.58 \\
9.54 \\
9.68 \\
0.68 \\
9.52 \\
0.29 \\
0.14\end{array}$ & $\begin{array}{l}9.63 \\
0.87 \\
9.92 \\
0.90 \\
0.82 \\
0.87 \\
0.36 \\
0.72 \\
0.69 \\
0.68 \\
0.72 \\
0.72 \\
0.62 \\
0.37 \\
0.16\end{array}$ & $\begin{array}{l}22.7 \\
20.6 \\
17.3\end{array}$ & $\begin{array}{l}49.49 \\
26.54 \\
19.37\end{array}$ & $\begin{array}{l}44.3 \\
48.2 \\
47.0\end{array}$ & $\begin{array}{l}1.91 \\
2.97 \\
2.55\end{array}$ & $\begin{array}{l}64.7 \\
62.4 \\
57.0\end{array}$ & $\begin{array}{l}0.42 \\
6.37 \\
0.30\end{array}$ \\
\hline
\end{tabular}

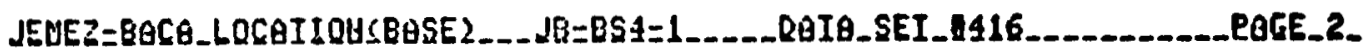
IMFEDAHCE TEHSOR

\begin{tabular}{|c|c|c|c|c|c|c|c|}
\hline PEF IOOO & $\operatorname{RE}(Z \mathrm{Q}: \mathrm{Y})$ & $I H(2 \times X)$ & RE(Z\%Y) & $I H(Z \not 2 y)$ & $\operatorname{RE}(Z Y X)$ & $\operatorname{IM}(Z Y X) \operatorname{RE}(Z Y Y)$ & $\operatorname{IIH}(Z Y Y)$ \\
\hline $\begin{array}{l}3000 \\
3900 \\
15000\end{array}$ & $\begin{array}{l}-0.1559 \\
-9.1179 \\
-0.9790\end{array}$ & $\begin{array}{l}-0.0130 \\
-0.0375 \\
-0.0540\end{array}$ & $\begin{array}{l}0.1579 \\
0.1454 \\
0.1638\end{array}$ & $\begin{array}{l}0.2036 \\
0.1892 \\
0.1747\end{array}$ & $\begin{array}{l}-0.0719 \\
-0.0662 \\
-0.0600\end{array}$ & $\begin{array}{rr}-0.0480 & 0.0143 \\
-0.0793 & -0.6981 \\
-0.0884 & -0.0132\end{array}$ & $\begin{array}{l}0.0433 \\
8.012 \epsilon \\
0.011 ?\end{array}$ \\
\hline
\end{tabular}


MAGNETOTELIURIC PARAMETERS FROM

JEMEZ MOUNTAINS SATELLITE SITES

\begin{tabular}{|c|c|c|c|c|c|c|c|c|c|}
\hline PERIOR & NU & $\begin{array}{l}\text { FRED } \\
\text { EY }\end{array}$ & $\begin{array}{c}\text { PRED } \\
\text { EY }\end{array}$ & $\begin{array}{l}\text { ANGLE } \\
(C W)\end{array}$ & RES-MAX & $\begin{array}{l}\text { PHASE } \\
\text { - MAX }\end{array}$ & RES-MIH & $\begin{array}{l}\text { PHASE } \\
\text { HIH }\end{array}$ & SKEH \\
\hline 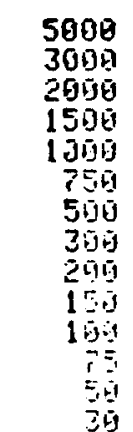 & $\begin{array}{r}8 \\
13 \\
8 \\
20 \\
25 \\
28 \\
25 \\
70 \\
190 \\
130 \\
190 \\
73 \\
430 \\
320\end{array}$ & $\begin{array}{l}0.90 \\
0.99 \\
0.86 \\
0.94 \\
6.97 \\
0.97 \\
0.95 \\
0.96 \\
9.95 \\
0.94 \\
9.91 \\
9.89 \\
0.97\end{array}$ & $\begin{array}{l}0.51 \\
0.51 \\
0.27 \\
0.54 \\
0.47 \\
0.59 \\
0.67 \\
0.82 \\
9.87 \\
0.90 \\
8.99 \\
0.89 \\
0.79 \\
0.74\end{array}$ & $\begin{array}{l}19.8 \\
15.8 \\
22.1 \\
24.7 \\
28.7 \\
30.8 \\
35.3 \\
40.2 \\
39.6\end{array}$ & $\begin{array}{l}55.81 \\
39.84 \\
31.51 \\
27.20 \\
26.85 \\
24.64 \\
20.83 \\
16.82 \\
15.73\end{array}$ & $\begin{array}{l}33.6 \\
42.2 \\
44.4 \\
39.3 \\
50.1 \\
54.2 \\
53.1 \\
52.8 \\
52.4\end{array}$ & $\begin{array}{l}1.09 \\
8.33 \\
0.12 \\
0.65 \\
0.97 \\
6.14 \\
0.58 \\
0.59 \\
0.41\end{array}$ & $\begin{array}{r}5.6 \\
17.1 \\
-8.1 \\
9.6 \\
83.9 \\
129.0 \\
154.4 \\
-173.7 \\
-177.7\end{array}$ & $\begin{array}{l}0.44 \\
0.65 \\
8.59 \\
0.57 \\
0.79 \\
8.84 \\
1.92 \\
1.24 \\
1.18\end{array}$ \\
\hline
\end{tabular}

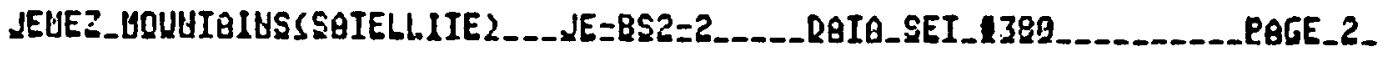

\begin{tabular}{|c|c|c|c|c|c|c|c|}
\hline \multicolumn{8}{|c|}{ IMPEDANCE TEHSOR } \\
\hline PERIOO & $R E(Z X X)$ & $I M(Z X X)$ & $\operatorname{RE}(Z Y Y Y)$ & $\operatorname{IH}(Z X Y) \operatorname{RE}(Z Y K)$ & $\operatorname{IM}(Z Y X)$ & RE (ZYY) & $\operatorname{IH}(Z Y Y)$ \\
\hline $\begin{array}{l}5000 \\
1500 \\
1909 \\
750 \\
500 \\
300 \\
290 \\
150 \\
100 \\
75\end{array}$ & $\begin{array}{l}-0.3048 \\
-0.1431 \\
-0.1851 \\
-0.2941 \\
-0.2493 \\
-0.3787 \\
-0.4543 \\
-0.6048 \\
-0.7201 \\
-0.7966\end{array}$ & $\begin{array}{l}-0.1814 \\
-0.1734 \\
-0.1846 \\
-0.2080 \\
-0.2800 \\
-0.3916 \\
-0.4932 \\
-0.5414 \\
-0.7137 \\
-0.7617\end{array}$ & $\begin{array}{r}-0.1731 \\
0.3387 \\
0.3048 \\
0.2876 \\
0.3649 \\
0.3349 \\
0.3203 \\
0.2494 \\
0.2355 \\
0.3063\end{array}$ & $\begin{array}{r}-0.4962-0.1164 \\
0.1999-0.6806 \\
0.2769-0.0618 \\
0.2733-0.0677 \\
0.2415-0.0606 \\
0.3995-0.0978 \\
0.5095-0.1922 \\
0.4940-0.1258 \\
0.4352-0.1483 \\
0.4972-0.1593\end{array}$ & $\begin{array}{l}-0.0469 \\
-0.0441 \\
-0.0317 \\
-0.0476 \\
-0.0927 \\
-0.1487 \\
-0.1812 \\
-0.2153 \\
-0.2767 \\
-0.3071\end{array}$ & $\begin{array}{r}-0.0443 \\
0.0123 \\
-0.9297 \\
-0.0910 \\
-0.0061 \\
-0.0256 \\
-0.0979 \\
-0.0051 \\
-0.0002 \\
-0.0181\end{array}$ & $\begin{array}{r}-0.1998 \\
0.0064 \\
-0.0401 \\
0.0282 \\
0.0233 \\
0.0151 \\
0.0154 \\
-0.0957 \\
0.0196 \\
0.0336\end{array}$ \\
\hline
\end{tabular}



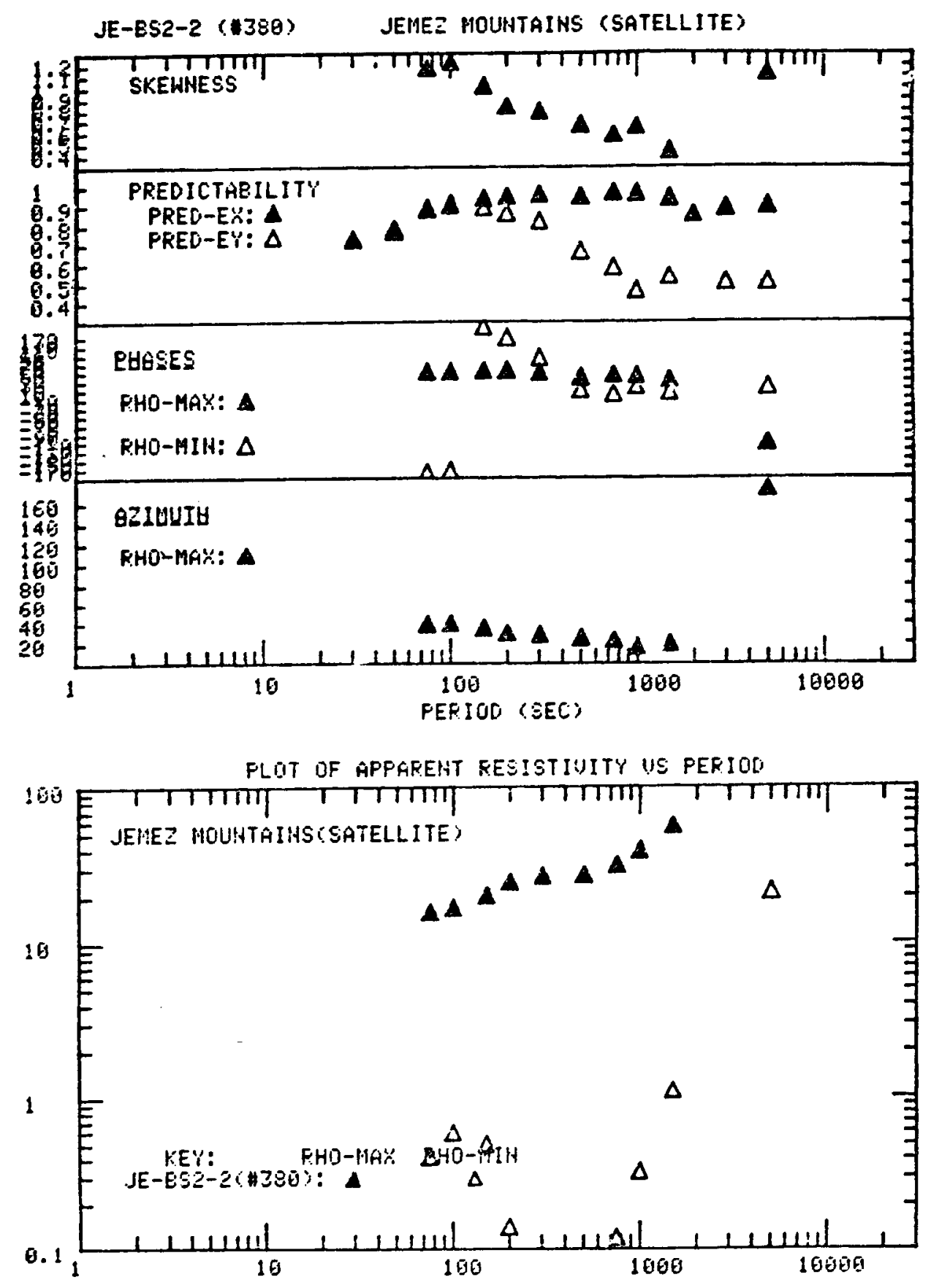


\begin{tabular}{|c|c|c|c|c|c|c|c|c|c|}
\hline PER IOD & HUI & $\begin{array}{c}\text { PRED } \\
\text { EX }\end{array}$ & $\underset{\text { EY }}{\text { PRED }}$ & $\begin{array}{l}\text { ANGLE } \\
\text { (CW) }\end{array}$ & RES-MAK & $\underset{\text { MAX }}{\text { PHASE }}$ & RES-MIH & $\begin{array}{l}\text { PHASE } \\
\text { MIIH }\end{array}$ & SKEH \\
\hline $\begin{array}{r}5069 \\
3009 \\
2009 \\
1509 \\
1096 \\
550 \\
509 \\
396 \\
200 \\
159 \\
195 \\
75 \\
59 \\
39 \\
29\end{array}$ & $\begin{array}{r}3 \\
5 \\
7 \\
10 \\
14 \\
19 \\
15 \\
38 \\
39 \\
54 \\
51 \\
129 \\
195 \\
251 \\
693\end{array}$ & $\begin{array}{l}0.92 \\
0.84 \\
0.87 \\
0.91 \\
0.85 \\
0.82 \\
0.89 \\
0.89 \\
0.86 \\
0.81 \\
0.68 \\
0.54 \\
0.41 \\
0.51 \\
0.45\end{array}$ & $\begin{array}{l}0.94 \\
0.87 \\
0.91 \\
0.94 \\
0.90 \\
0.87 \\
0.91 \\
0.92 \\
0.85 \\
0.78 \\
0.70 \\
0.58 \\
0.45 \\
0.52 \\
0.47\end{array}$ & $\begin{array}{r}175.3 \\
178.5 \\
64.5 \\
64.9 \\
57.1 \\
44.7 \\
47.9 \\
58.4 \\
55.4 \\
54.1\end{array}$ & $\begin{array}{r}326.26 \\
299.63 \\
70.95 \\
52.79 \\
39.83 \\
45.83 \\
36.65 \\
37.93 \\
24.56 \\
17.26\end{array}$ & $\begin{array}{r}125.5 \\
175.1 \\
61.3 \\
55.9 \\
55.7 \\
58.8 \\
51.6 \\
52.3 \\
57.7 \\
62.2\end{array}$ & $\begin{array}{r}112.45 \\
124.49 \\
18.76 \\
9.52 \\
5.17 \\
2.94 \\
5.25 \\
143.49 \\
0.21 \\
0.30\end{array}$ & $\begin{array}{r}-71.7 \\
36.5 \\
-151.0 \\
-152.1 \\
-69.8 \\
-0.6 \\
45.6 \\
49.3 \\
48.5 \\
64.5\end{array}$ & $\begin{array}{l}0.98 \\
2.28 \\
1.71 \\
1.77 \\
9.93 \\
0.47 \\
8.32 \\
0.52 \\
8.47 \\
0.51\end{array}$ \\
\hline
\end{tabular}

JEUEZ_UR!UIUIULSSSIELLIIE2

RGIO_SEI_L243.

BQGE_2.

\section{IMPEDANACE TENSOR}
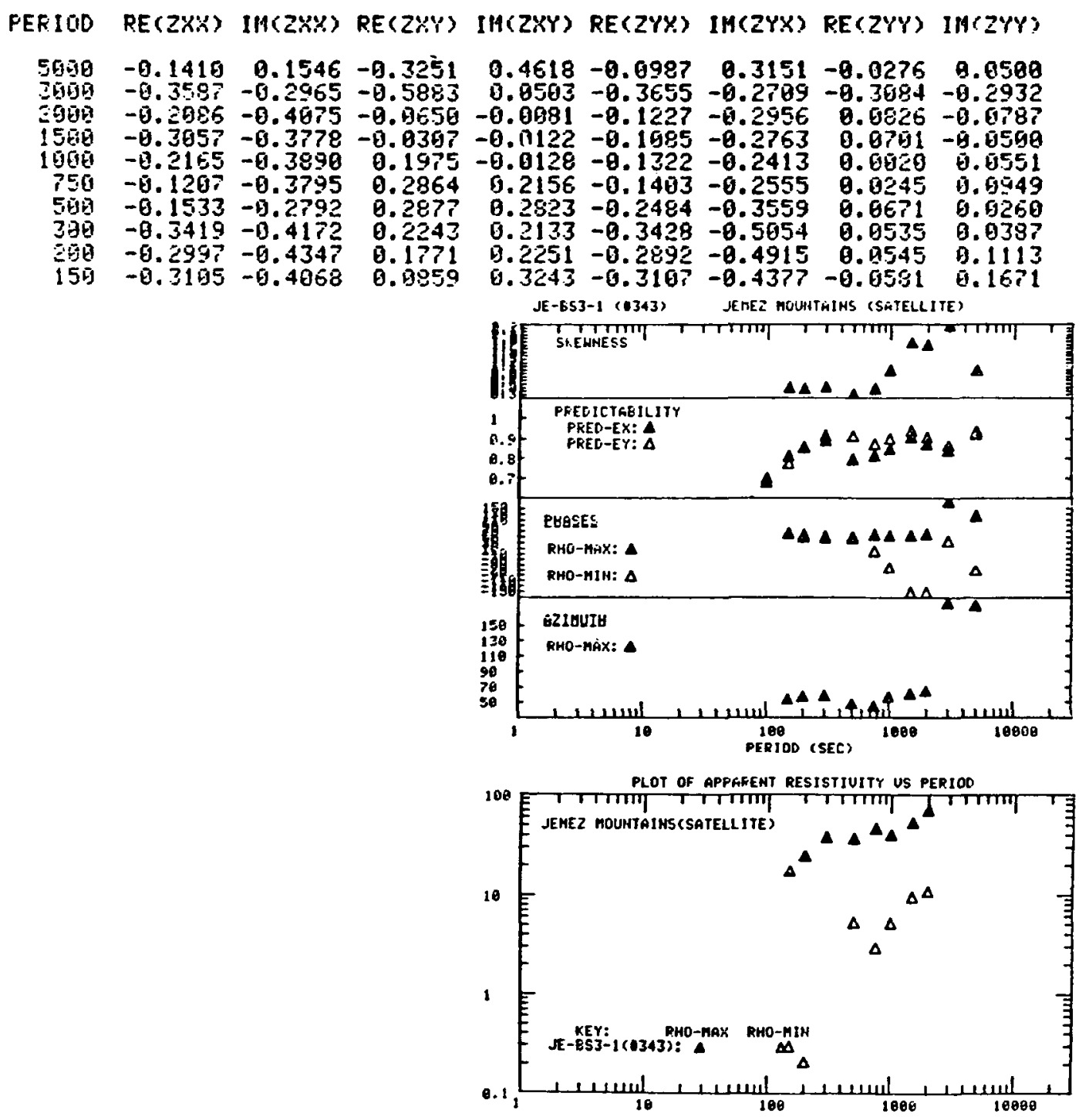


\begin{tabular}{|c|c|c|c|c|c|c|c|c|c|}
\hline FERIOD & HU & $\begin{array}{l}\text { PRED } \\
\text { EK. }\end{array}$ & $\begin{array}{l}\text { PRED } \\
\text { EY }\end{array}$ & $\begin{array}{l}\text { AHGLE } \\
\text { (CW) }\end{array}$ & FES-MAX & $\begin{array}{l}\text { PHASE } \\
\text { MAK }\end{array}$ & RES-MIN & $\underset{\text { HIN }}{\text { PHASE }}$ & SKEH \\
\hline 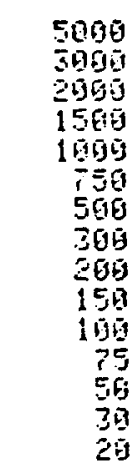 & $\begin{array}{r}3 \\
4 \\
E \\
8 \\
11 \\
15 \\
12 \\
39 \\
44 \\
70 \\
82 \\
92 \\
124 \\
255 \\
427\end{array}$ & $\begin{array}{l}1.09 \\
0.87 \\
0.98 \\
0.99 \\
0.96 \\
0.79 \\
0.71 \\
0.66 \\
0.68 \\
0.59 \\
0.71 \\
0.69 \\
0.53 \\
9.24 \\
0.10\end{array}$ & $\begin{array}{l}0.98 \\
0.99 \\
0.97 \\
9.90 \\
0.91 \\
0.82 \\
0.79 \\
0.68 \\
0.52 \\
0.53 \\
0.65 \\
0.65 \\
0.62 \\
0.28 \\
0.10\end{array}$ & $\begin{array}{l}49.7 \\
64.5 \\
66.1 \\
78.8 \\
75.2 \\
69.6\end{array}$ & $\begin{array}{r}180.96 \\
275.65 \\
57.65 \\
39.83 \\
18.47 \\
23.37\end{array}$ & $\begin{array}{l}98.2 \\
68.0 \\
44.9 \\
5 \epsilon .3 \\
60.9 \\
61.3\end{array}$ & $\begin{array}{r}120.31 \\
13.73 \\
17.58 \\
13.85 \\
14.42 \\
12.02\end{array}$ & $\begin{array}{r}-36.4 \\
151.6 \\
45.4 \\
42.8 \\
35.2 \\
31.1\end{array}$ & $\begin{array}{l}2.36 \\
1.12 \\
0.52 \\
0.47 \\
0.44 \\
6.39\end{array}$ \\
\hline
\end{tabular}

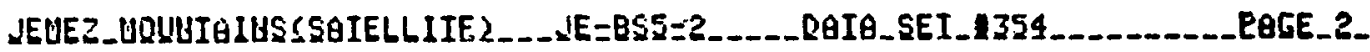

IHFEDAHCE TEHSOR

FEFITOS RE(ZYX) IM(ZYK) RE(ZKY) IM(ZKY) RE(ZYY) IH(ZYX) RE(ZYY) IH(ZYY)

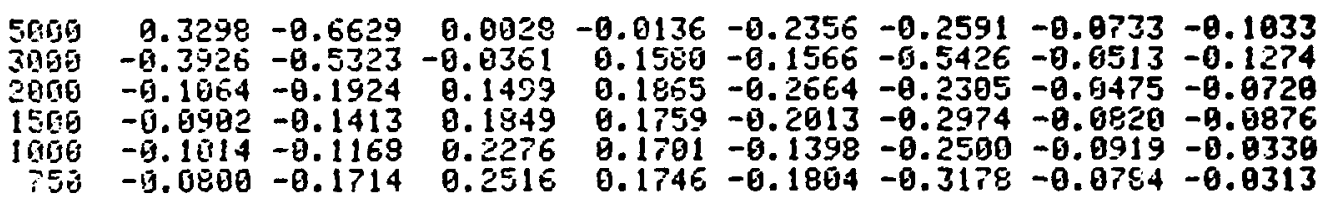



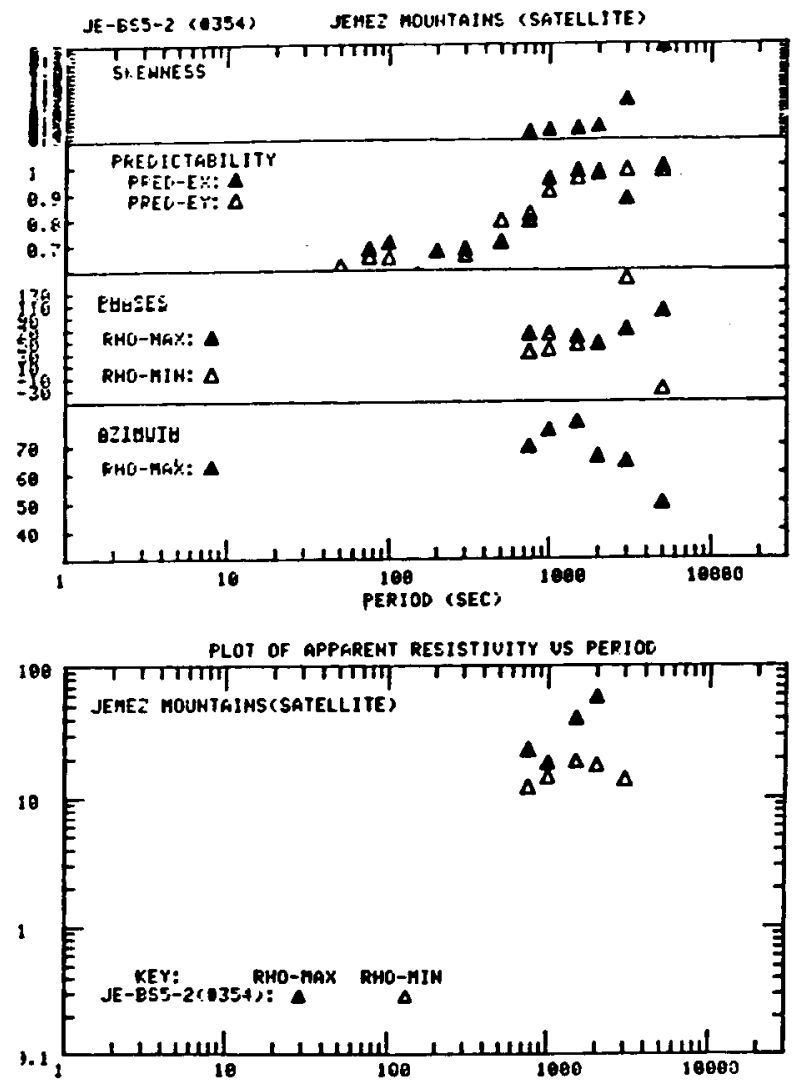

\begin{tabular}{|c|c|c|c|c|c|c|c|c|c|}
\hline PER IOQ, & HU & $\begin{array}{l}\text { PRED } \\
\text { EX }\end{array}$ & $\begin{array}{l}\text { PREO } \\
\text { EY }\end{array}$ & $\begin{array}{l}\text { ANGLE } \\
\text { (CH) }\end{array}$ & RES-MAX & $\begin{array}{l}\text { PHASE } \\
\text { MAAX }\end{array}$ & RES-HIH & $\begin{array}{l}\text { PHASE } \\
\text { HIH }\end{array}$ & SKEH \\
\hline \multirow{4}{*}{$\begin{array}{r}5009 \\
3001 \\
2000 \\
1590 \\
1000 \\
750 \\
500 \\
300 \\
200 \\
150 \\
190 \\
75 \\
50 \\
20\end{array}$} & \multirow{4}{*}{$\begin{array}{r}10 \\
16 \\
12 \\
15 \\
17 \\
25 \\
50 \\
99 \\
19 \\
197 \\
25 \\
247 \\
41 \\
11\end{array}$} & \multirow{4}{*}{$\begin{array}{l}0.86 \\
0.96 \\
0.91 \\
0.92 \\
0.90 \\
0.90 \\
0.81 \\
0.76 \\
0.81 \\
0.52 \\
0.93 \\
9.75 \\
9.913 \\
0.84\end{array}$} & \multirow{4}{*}{$\begin{array}{l}0.95 \\
0.99 \\
0.93 \\
0.95 \\
0.96 \\
0.94 \\
0.83 \\
0.71 \\
0.79 \\
0.55 \\
0.93 \\
0.86 \\
0.91 \\
0.80\end{array}$} & $\begin{array}{l}109.9 \\
115.9 \\
110.9 \\
107.6 \\
108.5 \\
109.1 \\
109.7\end{array}$ & $\begin{array}{l}179.23 \\
199.77 \\
173.13 \\
266.52 \\
242.81 \\
240.15 \\
178.53\end{array}$ & $\begin{array}{l}58.3 \\
55.0 \\
56.5 \\
54.0 \\
56.7 \\
56.7 \\
56.1\end{array}$ & $\begin{array}{l}6.17 \\
7.60 \\
8.85 \\
6.73 \\
3.99 \\
3.69 \\
2.47\end{array}$ & $\begin{array}{l}9.1 \\
31.2 \\
25.5 \\
21.1 \\
22.1 \\
25.2 \\
31.9\end{array}$ & $\begin{array}{l}0.16 \\
0.16 \\
0.18 \\
0.89 \\
0.07 \\
0.94 \\
0.82\end{array}$ \\
\hline & & & & 115.0 & 292.71 & 33.4 & 5.12 & 73.1 & 0.21 \\
\hline & & & & 187.1 & 370.29 & 50.9 & 7.09 & 61.7 & 0.03 \\
\hline & & & & $\begin{array}{r}96.4 \\
115.7\end{array}$ & $\begin{array}{r}330.92 \\
85.22\end{array}$ & $\begin{array}{r}47.8 \\
102.0\end{array}$ & $\begin{array}{l}0.97 \\
8.19\end{array}$ & $\begin{array}{l}71.6 \\
44.0\end{array}$ & $\begin{array}{l}0.25 \\
0.0\end{array}$ \\
\hline
\end{tabular}




\section{IMPEDAITCE TEHSOR}

PERIOD RE(ZKY) IIH(ZXY) RE(ZXY) IH(ZXY) RE(ZYX) IH(ZYX) RE(ZYY) IH(ZYY)

$\begin{array}{rrrr}5000 & 0.0257 & 0.0966 & 0.1085 \\ 3000 & 0.0584 & 0.1430 & 0.1616 \\ 2000 & 0.0562 & 0.1396 & 0.1803 \\ 1500 & 0.1137 & 0.1670 & 0.2958 \\ 1000 & 0.1272 & 0.2667 & 0.1973 \\ 750 & 0.2413 & 0.3282 & 0.2146 \\ 501 & 0.2238 & 0.3291 & 0.2210 \\ 260 & 0.6043 & 0.4725 & 0.5819 \\ 160 & 0.7835 & 0.7888 & 0.5220 \\ 50 & 1.2835 & 0.7878 & 0.1510 \\ 30 & 0.0291 & 0.5788 & -0.4863\end{array}$

$\begin{array}{lllll}0.0424 & -0.1907 & -0.3293 & -0.1033 & -0.0997 \\ 0.1216 & -0.2622 & -0.4067 & -0.1665 & -0.1626 \\ 0.1115 & -0.3173 & -0.5699 & -0.1270 & -0.1581 \\ 0.1021 & -0.4876 & -0.7146 & -0.2049 & -0.1945 \\ 0.1302 & -0.5380 & -0.8441 & -0.2078 & -0.2286 \\ 0.1540 & -0.6167 & -0.9643 & -0.2094 & -0.2629 \\ 0.1885 & -0.6578 & -1.0841 & -0.2087 & -0.2953 \\ 0.3696 & -1.4981 & -1.2878 & -0.8531 & -0.0045 \\ 0.7464 & -2.4717 & -3.1245 & -0.6643 & -0.7155 \\ 0.3401 & -3.8116 & -4.2175 & 0.3193 & -0.0453 \\ 0.7763 & -0.5452 & -3.7283 & 2.9232 & -0.7541\end{array}$

IE-ESE-1 (1035:

JEMEZ MOLNTGINS (SATELLITE)

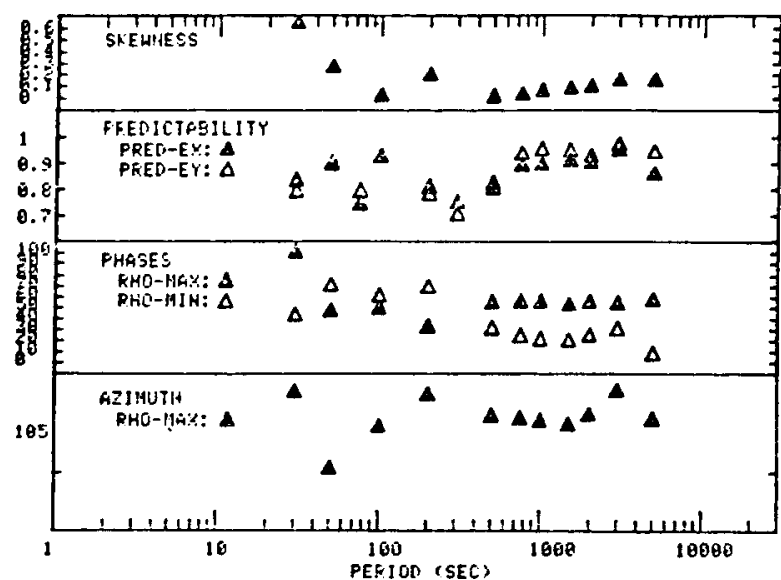

FLOT OF APFAREIT HESISTIUITY US PERIOD

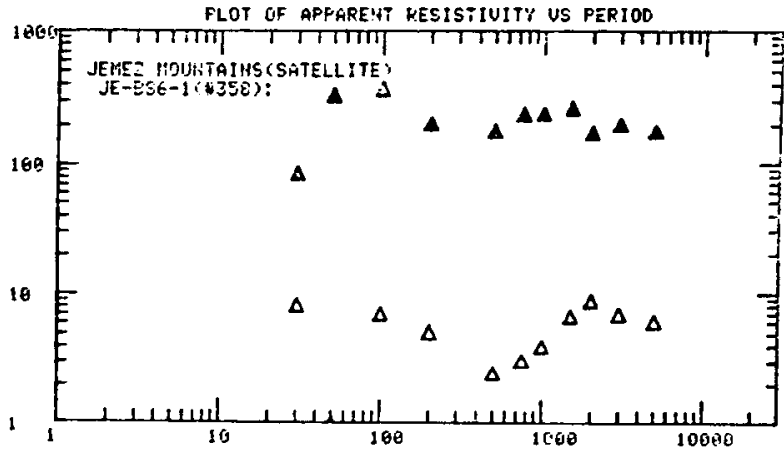




\begin{tabular}{|c|c|c|c|c|c|c|c|c|c|}
\hline PERIOD & HU & $\begin{array}{c}\text { PRED } \\
\text { E. }\end{array}$ & $\begin{array}{c}\text { PRED } \\
\text { EY }\end{array}$ & $\begin{array}{l}\text { ANGGLE } \\
\text { (CW) }\end{array}$ & RES-MAX & $\begin{array}{l}\text { PHASE } \\
\text {, HAAY }\end{array}$ & RES-MIN & $\underset{\text { PHIHE }}{\text { PHASE }}$ & SKEEH \\
\hline \multirow{2}{*}{$\begin{array}{r}10000 \\
7500 \\
5090 \\
3009 \\
2009 \\
1500 \\
1009 \\
750 \\
500 \\
300 \\
200 \\
150 \\
100 \\
75 \\
50 \\
30 \\
20\end{array}$} & \multirow[b]{2}{*}{$\begin{array}{r}506 \\
1000 \\
1378\end{array}$} & \multirow{2}{*}{$\begin{array}{l}0.99 \\
0.99 \\
0.92 \\
0.88 \\
0.97 \\
0.94 \\
0.92 \\
0.93 \\
0.89 \\
0.83 \\
0.78 \\
0.70 \\
0.51 \\
0.36 \\
0.24 \\
0.14 \\
0.08\end{array}$} & \multirow{2}{*}{$\begin{array}{l}0.59 \\
0.84 \\
0.65 \\
0.36 \\
0.81 \\
0.89 \\
0.88 \\
0.87 \\
0.76 \\
0.62 \\
0.57 \\
0.46 \\
0.32 \\
0.29 \\
0.15 \\
0.10 \\
0.11\end{array}$} & $\begin{array}{l}33.5 \\
24.1 \\
26.5\end{array}$ & $\begin{array}{l}45.11 \\
42.08 \\
34.27\end{array}$ & $\begin{array}{r}-6.7 \\
11.4 \\
1.4\end{array}$ & $\begin{array}{r}18.69 \\
9.68 \\
1.67\end{array}$ & $\begin{array}{r}171.8 \\
-148.8 \\
-175.3\end{array}$ & $\begin{array}{l}4.12 \\
1.35 \\
0.25\end{array}$ \\
\hline & & & & $\begin{array}{l}18.0 \\
19.3 \\
23.2 \\
21.0 \\
25.3\end{array}$ & $\begin{array}{r}17.11 \\
11.71 \\
10.35 \\
8.47 \\
5.38\end{array}$ & $\begin{array}{l}31.3 \\
32.8 \\
43.5 \\
46.5 \\
45.5\end{array}$ & $\begin{array}{l}2.98 \\
1.97 \\
1.59 \\
1.43 \\
1.29\end{array}$ & $\begin{array}{l}59.0 \\
54.6 \\
57.9 \\
58.9 \\
55.5\end{array}$ & $\begin{array}{l}0.26 \\
0.20 \\
0.21 \\
0.27 \\
0.25\end{array}$ \\
\hline
\end{tabular}

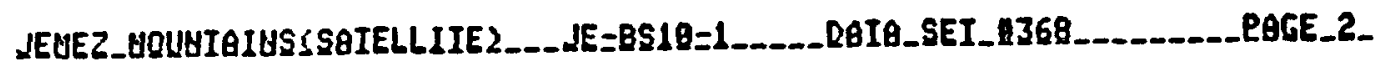

\begin{tabular}{|c|c|c|c|c|c|c|c|c|}
\hline RIOD & $E(2 \times x)$ & $\operatorname{IM}(2 K K)$ & $\operatorname{RE}(Z K Y)$ & $\operatorname{IM}(Z X Y)$ & $\operatorname{RE}(Z Y X)$ & $\operatorname{Im}(Z Y X)$ & RE (ZYY) & $\operatorname{IH}(Z Y Y)$ \\
\hline $\begin{array}{r}19900 \\
7500 \\
5000 \\
2000 \\
1509 \\
1000 \\
750 \\
590\end{array}$ & $\begin{array}{l}-0.0671 \\
-0.0626 \\
-0.0942 \\
-0.0619 \\
-0.0442 \\
-0.0504 \\
-0.0622 \\
-0.0560\end{array}$ & $\begin{array}{l}-0.0756 \\
-0.0730 \\
-0.0202 \\
-0.0479 \\
-0.0477 \\
-0.0661 \\
-0.0717 \\
-0.0745\end{array}$ & $\begin{array}{l}0.0781 \\
0.1205 \\
0.1401 \\
0.1652 \\
0.1558 \\
0.1503 \\
0.1550 \\
0.1595\end{array}$ & $\begin{array}{l}0.0199 \\
0.0359 \\
0.0632 \\
0.0978 \\
0.0958 \\
0.1386 \\
0.1535 \\
0.1323\end{array}$ & $\begin{array}{r}0.0244 \\
0.0245 \\
-0.6043 \\
-0.0486 \\
-0.0580 \\
-0.0619 \\
-0.6596 \\
-0.0675\end{array}$ & $\begin{array}{r}0.6236 \\
0.6454 \\
8.8021 \\
-0.0715 \\
-0.0775 \\
-0.0934 \\
-0.1024 \\
-0.1269\end{array}$ & $\begin{array}{r}0.1551 \\
8.1194 \\
8.8861 \\
0.8151 \\
0.0234 \\
8.0279 \\
8.0004 \\
-0.8044\end{array}$ & $\begin{array}{r}-0.1279 \\
-0.0442 \\
-8.0143 \\
-0.0061 \\
-0.0033 \\
0.0030 \\
8.0045 \\
0.0142\end{array}$ \\
\hline
\end{tabular}



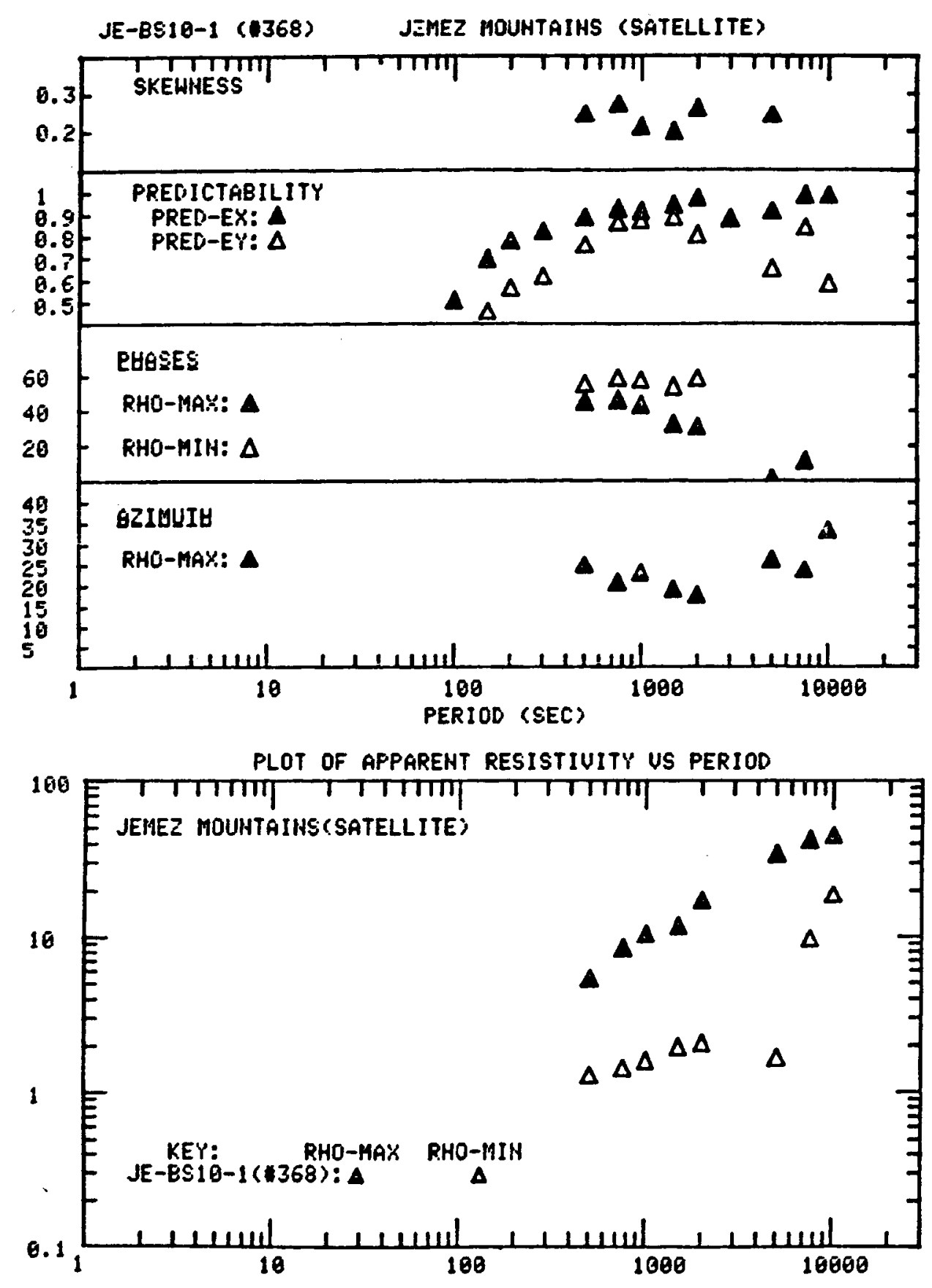


\begin{tabular}{|c|c|c|c|c|c|c|c|c|c|}
\hline PERIOD & NU & $\begin{array}{c}\text { PRED } \\
\text { EX }\end{array}$ & $\begin{array}{c}\text { PRED } \\
\text { EY }\end{array}$ & $\begin{array}{l}\text { ANGLEE } \\
\text { (CW) }\end{array}$ & RES-MAX & $\underset{\text { HAK }}{\text { PHASE }}$ & RES-MIH & $\begin{array}{l}\text { PHASE } \\
\text { MIN }\end{array}$ & SKEH \\
\hline $\begin{array}{r}5000 \\
3000 \\
3080 \\
2900 \\
2900 \\
1500 \\
1590 \\
1090 \\
1009 \\
750 \\
750 \\
500 \\
500 \\
300 \\
360 \\
309 \\
300\end{array}$ & $\begin{array}{l}10 \\
16 \\
11 \\
16 \\
10 \\
15 \\
11 \\
26 \\
24 \\
28 \\
24 \\
38 \\
66 \\
75 \\
52 \\
15 \\
185\end{array}$ & $\begin{array}{l}0.87 \\
0.81 \\
0.95 \\
0.98 \\
0.92 \\
0.89 \\
0.95 \\
0.90 \\
0.82 \\
0.75 \\
0.86 \\
0.87 \\
0.84 \\
0.78 \\
0.82 \\
0.92 \\
0.73\end{array}$ & $\begin{array}{l}0.88 \\
0.91 \\
0.99 \\
0.99 \\
0.95 \\
0.97 \\
0.99 \\
0.98 \\
0.96 \\
0.94 \\
0.97 \\
0.95 \\
0.92 \\
0.83 \\
0.86 \\
0.96 \\
0.78\end{array}$ & $\begin{array}{r}155.2 \\
156.6 \\
157.1 \\
165.2 \\
164.1 \\
159.8 \\
160.6 \\
158.4 \\
156.3 \\
159.6 \\
159.8 \\
161.9 \\
160.8 \\
156.2 \\
158.8 \\
48.3\end{array}$ & $\begin{array}{r}32.23 \\
16.17 \\
18.59 \\
26.88 \\
23.77 \\
17.28 \\
19.81 \\
12.98 \\
12.27 \\
10.11 \\
11.72 \\
12.36 \\
11.37 \\
8.08 \\
7.84 \\
16.18\end{array}$ & $\begin{array}{l}47.6 \\
42.7 \\
47.8 \\
35.9 \\
36.5 \\
38.4 \\
37.5 \\
31.8 \\
35.7 \\
31.7 \\
35.8 \\
48.5 \\
46.6 \\
47.6 \\
48.8 \\
42.8\end{array}$ & $\begin{array}{l}1.69 \\
0.49 \\
1.38 \\
1.25 \\
1.22 \\
1.54 \\
1.42 \\
1.28 \\
1.38 \\
2.07 \\
2.05 \\
1.88 \\
1.75 \\
1.23 \\
1.64 \\
4.72\end{array}$ & $\begin{array}{l}45.6 \\
59.1 \\
54.6 \\
63.6 \\
62.9 \\
61.4 \\
58.7 \\
51.0 \\
57.4 \\
62.7 \\
60.1 \\
E 5.3 \\
64.4 \\
64.2 \\
E 3.8 \\
35.4\end{array}$ & $\begin{array}{l}0.66 \\
0.96 \\
0.63 \\
0.55 \\
0.61 \\
0.54 \\
0.51 \\
0.56 \\
0.57 \\
0.56 \\
0.58 \\
0.47 \\
0.48 \\
0.59 \\
0.54 \\
1.14\end{array}$ \\
\hline $\begin{array}{r}100 \\
75 \\
50 \\
39 \\
20\end{array}$ & $\begin{array}{r}147 \\
17 \\
235 \\
317 \\
842 \\
1233\end{array}$ & $\begin{array}{l}0.10 \\
0.97 \\
0.69 \\
0.43 \\
0.17 \\
0.10\end{array}$ & $\begin{array}{l}0.79 \\
0.97 \\
0.76 \\
0.51 \\
0.18 \\
0.08\end{array}$ & 160.1 & 13.63 & 45.4 & 3.27 & 81.0 & 0.56 \\
\hline
\end{tabular}

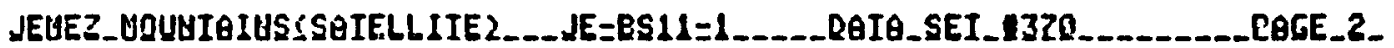
IMPEDAHCE TENSOR

\begin{tabular}{|c|c|c|c|c|c|c|c|c|}
\hline & $\operatorname{RE}(2 \times \%)$ & $\operatorname{IM}(2 X X)$ & $\operatorname{RE}(Z K Y)$ & $\operatorname{IH}(Z X Y)$ & $\operatorname{RE}(Z Y X)$ & $\operatorname{IA}(Z Y X)$ & RE (ZYY) & $\operatorname{IM}(Z Y Y)$ \\
\hline $\begin{array}{l}5000 \\
3800 \\
3000 \\
2080 \\
2800 \\
1500 \\
1500 \\
1009 \\
1009 \\
759 \\
759 \\
500 \\
590 \\
369 \\
309 \\
309 \\
109\end{array}$ & $\begin{array}{l}-0.0252 \\
-0.0478 \\
-0.0301 \\
-0.0193 \\
-0.0256 \\
-0.6270 \\
-0.0278 \\
-0.0392 \\
-0.0368 \\
-0.0426 \\
-0.0593 \\
-0.9530 \\
-0.0503 \\
-0.0311 \\
-0.0616 \\
-0.4171 \\
-0.1933\end{array}$ & $\begin{array}{r}-0.0088 \\
-0.0012 \\
-0.6846 \\
-0.0292 \\
-0.0295 \\
-0.6018 \\
0.0076 \\
0.0233 \\
0.0123 \\
-0.8072 \\
-0.0136 \\
-0.0033 \\
-0.8028 \\
-0.0225 \\
-0.0367 \\
-0.3956 \\
0.0388\end{array}$ & $\begin{array}{l}0.1022 \\
0.1043 \\
0.1113 \\
0.1982 \\
0.1828 \\
0.1712 \\
0.1907 \\
6.2045 \\
0.1845 \\
0.2046 \\
6.2107 \\
0.2167 \\
0.2142 \\
0.2683 \\
6.2114 \\
0.3546 \\
0.5306\end{array}$ & $\begin{array}{l}0.1167 \\
0.0572 \\
0.1099 \\
0.1450 \\
0.1395 \\
0.1328 \\
0.1364 \\
0.0944 \\
0.1142 \\
0.1119 \\
0.1460 \\
0.2491 \\
0.2294 \\
0.2597 \\
0.2629 \\
0.2060 \\
0.5573\end{array}$ & $\begin{array}{l}-0.0476 \\
-0.0311 \\
-0.0348 \\
-0.0368 \\
-0.0382 \\
-0.0512 \\
-0.0489 \\
-0.0641 \\
-0.0613 \\
-0.0703 \\
-0.0743 \\
-0.0735 \\
-0.0746 \\
-0.1016 \\
-0.1006 \\
-0.2548 \\
-0.1807\end{array}$ & $\begin{array}{l}-0.0453 \\
-0.0388 \\
-0.0597 \\
-0.0570 \\
-0.0558 \\
-0.0792 \\
-0.0787 \\
-0.0992 \\
-0.1002 \\
-0.1289 \\
-0.1248 \\
-0.1390 \\
-0.1351 \\
-0.1399 \\
-0.1573 \\
-0.3152 \\
-0.4131\end{array}$ & $\begin{array}{r}-0.1000 \\
-0.1244 \\
0.6817 \\
-0.1102 \\
-0.1163 \\
-0.1236 \\
-0.1200 \\
-0.1364 \\
-0.1362 \\
-0.1426 \\
-0.1504 \\
-0.1548 \\
-0.1548 \\
-0.1945 \\
-0.1947 \\
-0.2525 \\
-0.4723\end{array}$ & $\begin{array}{l}-0.0755 \\
-0.0653 \\
-0.0831 \\
-0.0891 \\
-0.0783 \\
-0.0709 \\
-0.0765 \\
-0.0790 \\
-0.0762 \\
-0.0760 \\
-0.0998 \\
-0.0876 \\
-0.0864 \\
-0.1055 \\
-0.1044 \\
-0.2169 \\
-0.1093\end{array}$ \\
\hline
\end{tabular}



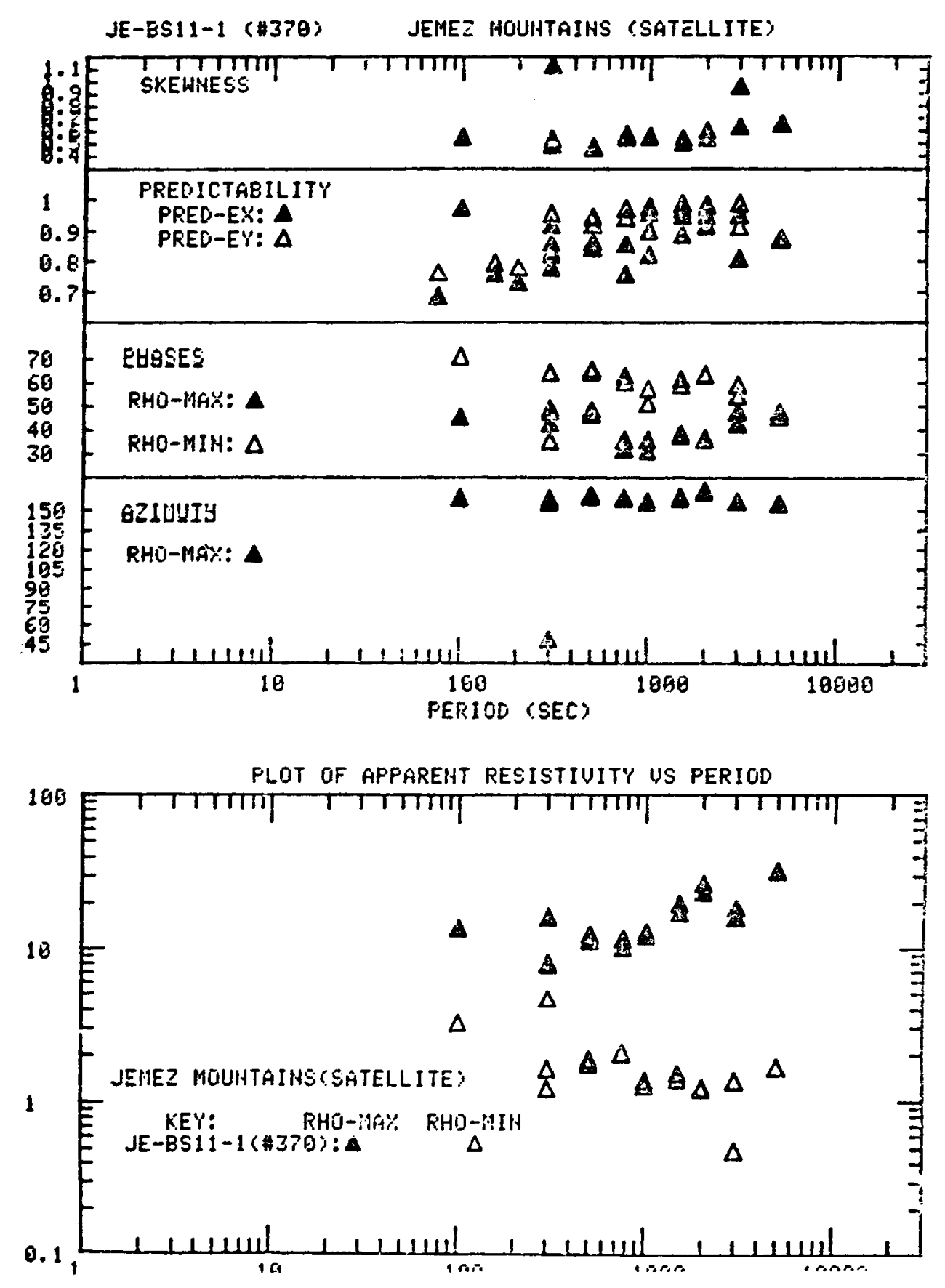


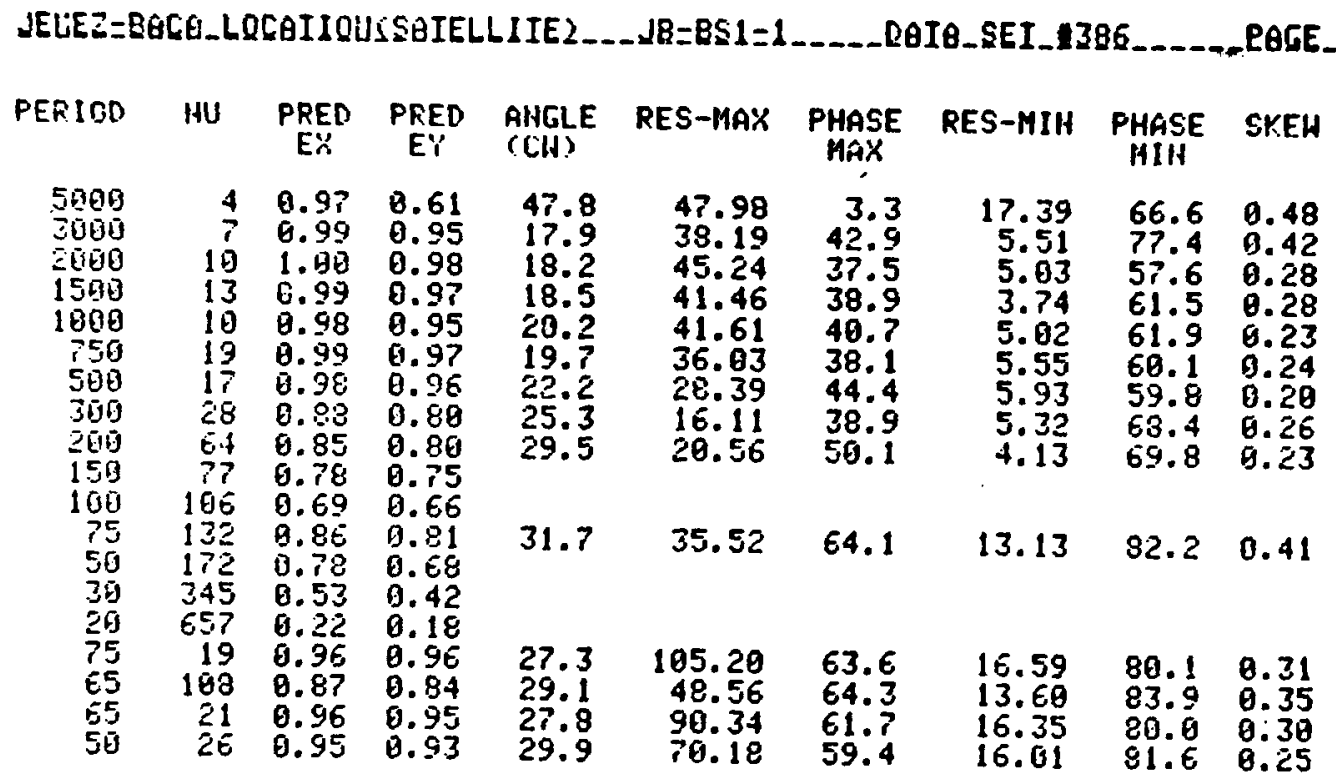

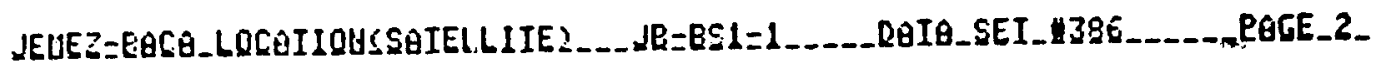

IMFELAHCE TENSOR

\begin{tabular}{|c|c|c|c|c|c|c|c|c|}
\hline 0100 & RE $(2 \times x)$ & $I H(2 X X)$ & $R E(Z X Y)$ & II & $\operatorname{RE}(Z Y Y)$ & $\operatorname{IH}(Z Y X)$ & $R E(Z Y Y)$ & IM(ZYY) \\
\hline $\begin{array}{l}5900 \\
3090 \\
2009 \\
1509 \\
1909 \\
750 \\
590 \\
300 \\
200 \\
75 \\
75 \\
65 \\
65 \\
50\end{array}$ & $\begin{array}{l}-0.1555 \\
-0.1230 \\
-0.1120 \\
-0.1149 \\
-0.1431 \\
-0.1462 \\
-0.1182 \\
-0.1588 \\
-0.1931 \\
-0.1712 \\
-0.8393 \\
-0.8368 \\
-0.9200 \\
-0.9538\end{array}$ & $\begin{array}{r}0.0643 \\
0.0431 \\
-0.0686 \\
-0.0980 \\
-0.0942 \\
-0.1257 \\
-0.1554 \\
-0.0975 \\
-0.2299 \\
-0.1934 \\
-0.1559 \\
-0.2567 \\
-0.6968 \\
-0.5754\end{array}$ & $\begin{array}{l}0.1435 \\
0.1593 \\
0.2487 \\
0.2731 \\
0.3233 \\
0.3672 \\
0.3714 \\
0.3563 \\
0.4940 \\
9.4616 \\
1.1275 \\
0.6455 \\
1.1157 \\
1.0749\end{array}$ & $\begin{array}{l}0.9969 \\
0.1859 \\
0.1901 \\
0.2070 \\
0.2619 \\
0.2562 \\
0.3061 \\
0.2765 \\
0.4498 \\
1.3290 \\
1.9718 \\
1.5969 \\
1.9728 \\
2.0088\end{array}$ & $\begin{array}{l}-0.1275 \\
-0.0466 \\
-0.0783 \\
-0.0697 \\
-0.0971 \\
-0.1144 \\
-0.1320 \\
-0.1567 \\
-0.1670 \\
-0.3373 \\
-0.2299 \\
-0.3616 \\
-0.3285 \\
-0.4583\end{array}$ & $\begin{array}{l}-0.0368 \\
-0.0792 \\
-0.1692 \\
-0.1243 \\
-0.1754 \\
-0.2129 \\
-0.2769 \\
-0.3254 \\
-0.4014 \\
-0.9827 \\
-1.4369 \\
-1.1680 \\
-1.4533 \\
-1.5235\end{array}$ & $\begin{array}{r}0.0133 \\
0.0013 \\
0.0054 \\
0.0076 \\
0.0093 \\
0.0091 \\
0.0662 \\
0.0171 \\
0.0722 \\
-0.2257 \\
-0.0540 \\
-0.1931 \\
-0.1826 \\
0.0331\end{array}$ & $\begin{array}{r}-0.0388 \\
0.028 \epsilon \\
0.0069 \\
0.0170 \\
0.0478 \\
0.0350 \\
0.0471 \\
0.0057 \\
0.0306 \\
0.1553 \\
0.5011 \\
0.3335 \\
0.4285 \\
0.3330\end{array}$ \\
\hline
\end{tabular}



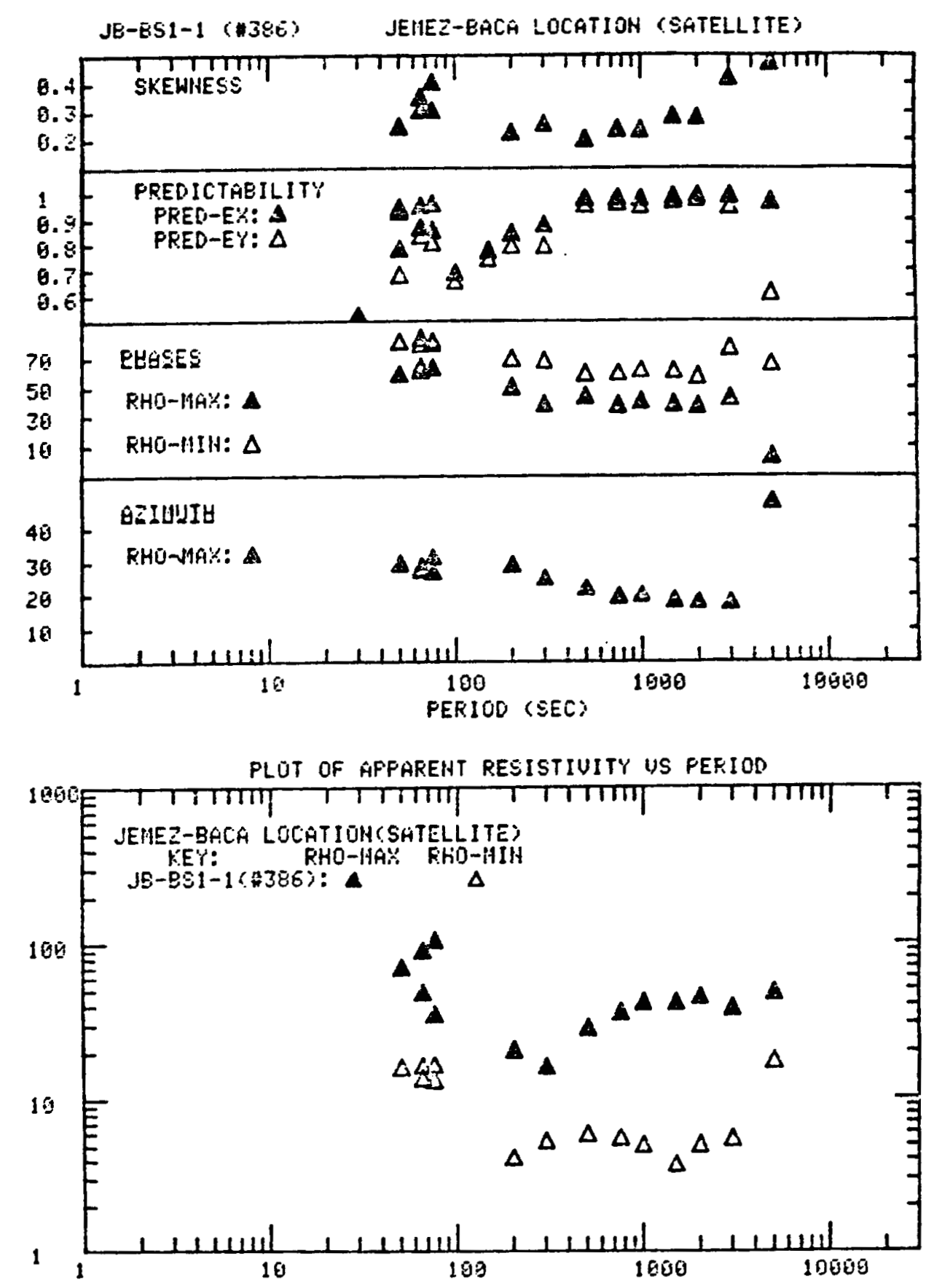


\begin{tabular}{|c|c|c|c|c|c|c|c|c|c|}
\hline PERIOD & HU & $\begin{array}{l}\text { PRED } \\
\text { EK }\end{array}$ & $\begin{array}{l}\text { PRED } \\
\text { EY }\end{array}$ & $\begin{array}{l}\text { AHCLEE } \\
\text { (CW) }\end{array}$ & RES-MAY & $\underset{\text { MAX }}{\text { PHASE }}$ & RES MIN & $\begin{array}{l}\text { PHASE } \\
\text { MIH }\end{array}$ & SKEH \\
\hline $\begin{array}{l}5000 \\
3005 \\
2009 \\
1509 \\
1090 \\
750 \\
500 \\
300 \\
200 \\
150 \\
190 \\
75 \\
50 \\
30 \\
20\end{array}$ & $\begin{array}{r}7 \\
11 \\
17 \\
16 \\
16 \\
21 \\
41 \\
44 \\
91 \\
147 \\
246 \\
324 \\
385 \\
634 \\
1286\end{array}$ & $\begin{array}{l}0.99 \\
0.98 \\
0.99 \\
0.98 \\
0.93 \\
0.92 \\
0.90 \\
0.83 \\
0.75 \\
0.57 \\
0.61 \\
0.71 \\
0.68 \\
0.38 \\
0.23\end{array}$ & $\begin{array}{l}0.87 \\
0.81 \\
0.87 \\
0.98 \\
0.75 \\
0.63 \\
0.63 \\
0.25 \\
0.29 \\
0.37 \\
0.47 \\
0.50 \\
0.38 \\
0.16 \\
0.12\end{array}$ & $\begin{array}{r}178.6 \\
0.7 \\
4.3 \\
2.0 \\
169.4 \\
174.3 \\
170.9\end{array}$ & $\begin{array}{l}27.17 \\
25.68 \\
22.52 \\
19.05 \\
24.17 \\
18.16 \\
12.95\end{array}$ & $\begin{array}{l}34.4 \\
35.8 \\
37.5 \\
46.8 \\
46.2 \\
47.1 \\
47.9\end{array}$ & $\begin{array}{l}1.54 \\
0.65 \\
0.96 \\
0.61 \\
1.11 \\
1.03 \\
2.71\end{array}$ & $\begin{array}{l}83.8 \\
47.8 \\
63.7 \\
66.5 \\
49.1 \\
66.8 \\
66.8\end{array}$ & $\begin{array}{l}0.07 \\
0.23 \\
0.26 \\
0.27 \\
0.36 \\
0.33 \\
9.32\end{array}$ \\
\hline
\end{tabular}

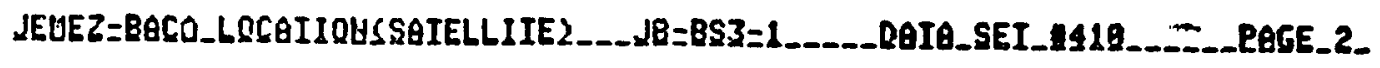

IHPEDANCE TENSOR

\begin{tabular}{|c|c|c|c|c|c|c|c|c|}
\hline 100 & $(Z K K)$ & $\operatorname{IH}(2 X X)$ & $\operatorname{RE}(Z X Y)$ & $\operatorname{IM}(Z X Y)$ & RE (ZYX) & $\operatorname{IH}(2 Y X)$ & $\operatorname{RE}(Z Y Y)$ & IH(ZYY) \\
\hline $\begin{array}{l}5000 \\
3960 \\
2000 \\
1500 \\
1000 \\
759 \\
500\end{array}$ & $\begin{array}{r}0.01971 \\
0.0190 \\
-0.0141 \\
0.0048 \\
0.1021 \\
0.0630 \\
0.0892\end{array}$ & $\begin{array}{l}0.0094 \\
0.0169 \\
0.0419 \\
0.0333 \\
0.0741 \\
0.0748 \\
0.0733\end{array}$ & $\begin{array}{l}0.1360 \\
0.1675 \\
0.1861 \\
0.1906 \\
0.2315 \\
0.2358 \\
0.2407\end{array}$ & $\begin{array}{l}0.09 \\
0.12 \\
0.14 \\
0.16 \\
0.24 \\
0.25 \\
0.25\end{array}$ & $\begin{array}{l}-0 . \\
-0 . \\
-8 . \\
-0 . \\
-0 . \\
-8 . \\
-0 .\end{array}$ & $\begin{array}{l}-0 . \\
-6 . \\
-6 . \\
-0 . \\
-6 . \\
-6 . \\
-6 .\end{array}$ & $\begin{array}{l}0.0873 \\
8.0201 \\
0.0276 \\
0.0172 \\
0.0184 \\
0.0351 \\
0.0575\end{array}$ & $\begin{array}{r}-0.08 \\
0.02 \\
0.02 \\
0.0 \\
0.01 \\
0.02 \\
0.06\end{array}$ \\
\hline
\end{tabular}
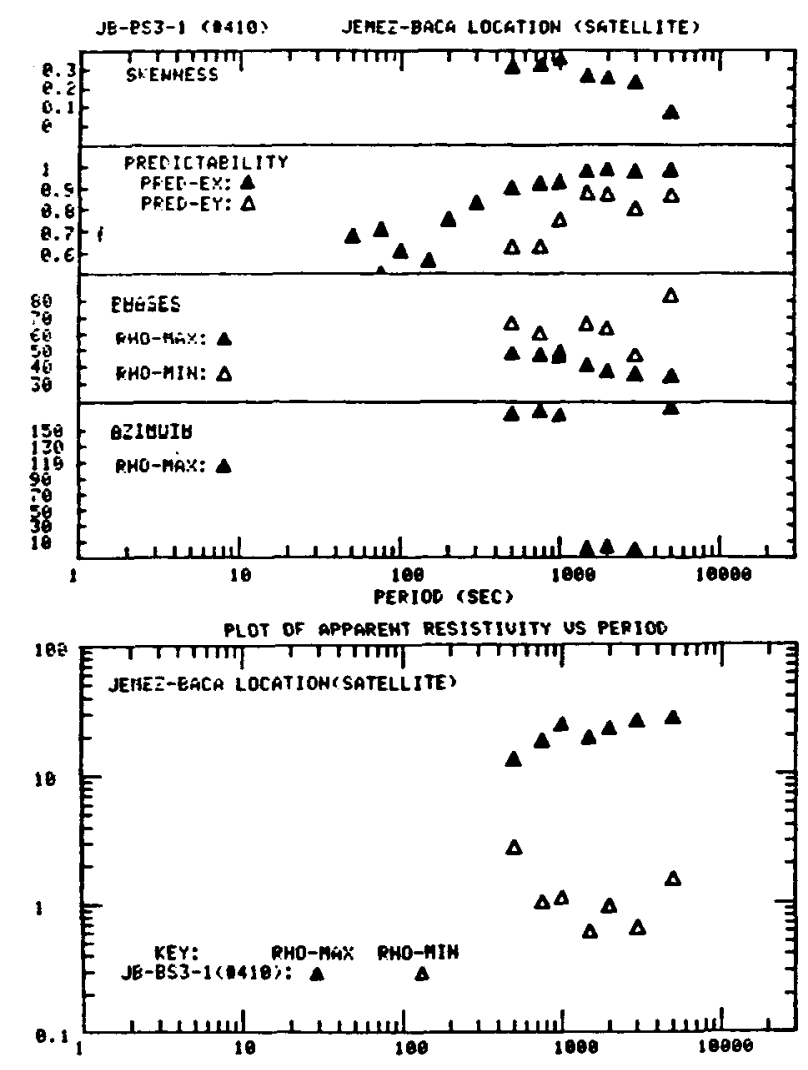


\begin{tabular}{|c|c|c|c|c|c|c|c|c|c|}
\hline PERIOD & HU & $\begin{array}{c}\text { PRED } \\
\text { EX }\end{array}$ & $\begin{array}{l}\text { PREO } \\
\text { EY }\end{array}$ & $\begin{array}{l}\text { AHGLE } \\
\text { (CW) }\end{array}$ & RES-HAX & $\begin{array}{l}\text { PHASE } \\
\text { MAX }\end{array}$ & RES-IIIIH & $\begin{array}{l}\text { PHASE } \\
\text { MIH }\end{array}$ & SKEH \\
\hline $\begin{array}{r}5000 \\
3009 \\
2090 \\
1509 \\
1090 \\
750 \\
504 \\
700 \\
200 \\
150 \\
190 \\
75 \\
50 \\
30 \\
20\end{array}$ & $\begin{array}{r}10 \\
16 \\
13 \\
19 \\
21 \\
26 \\
45 \\
70 \\
144 \\
201 \\
346 \\
431 \\
599 \\
1183 \\
1823\end{array}$ & $\begin{array}{l}0.57 \\
0.90 \\
0.87 \\
0.91 \\
0.94 \\
9.91 \\
0.94 \\
0.69 \\
0.46 \\
0.39 \\
9.49 \\
9.46 \\
9.31 \\
9.17 \\
0.07\end{array}$ & $\begin{array}{l}0.66 \\
0.36 \\
0.62 \\
0.76 \\
0.69 \\
0.57 \\
0.65 \\
0.49 \\
0.27 \\
9.41 \\
9.48 \\
0.38 \\
9.33 \\
0.14 \\
0.97\end{array}$ & $\begin{array}{r}15.9 \\
14.8 \\
7.2\end{array}$ & $\begin{array}{l}22.67 \\
17.79 \\
13.91\end{array}$ & $\begin{array}{l}34.1 \\
33.9 \\
36.9\end{array}$ & $\begin{array}{l}2.71 \\
3.97 \\
5.64\end{array}$ & $\begin{array}{l}75.8 \\
6 E .6 \\
54.4\end{array}$ & $\begin{array}{l}0.62 \\
6.35 \\
0.27\end{array}$ \\
\hline
\end{tabular}

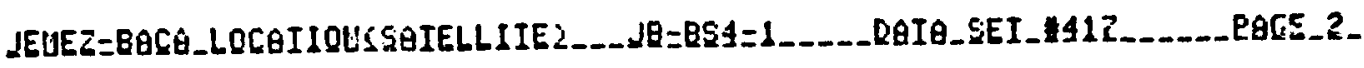

IAPEDHHCE TEHSOR

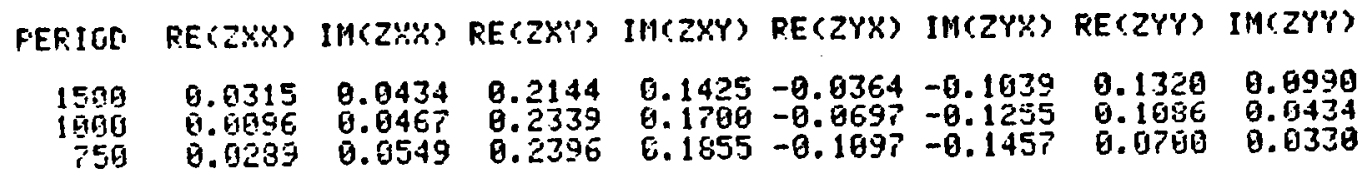
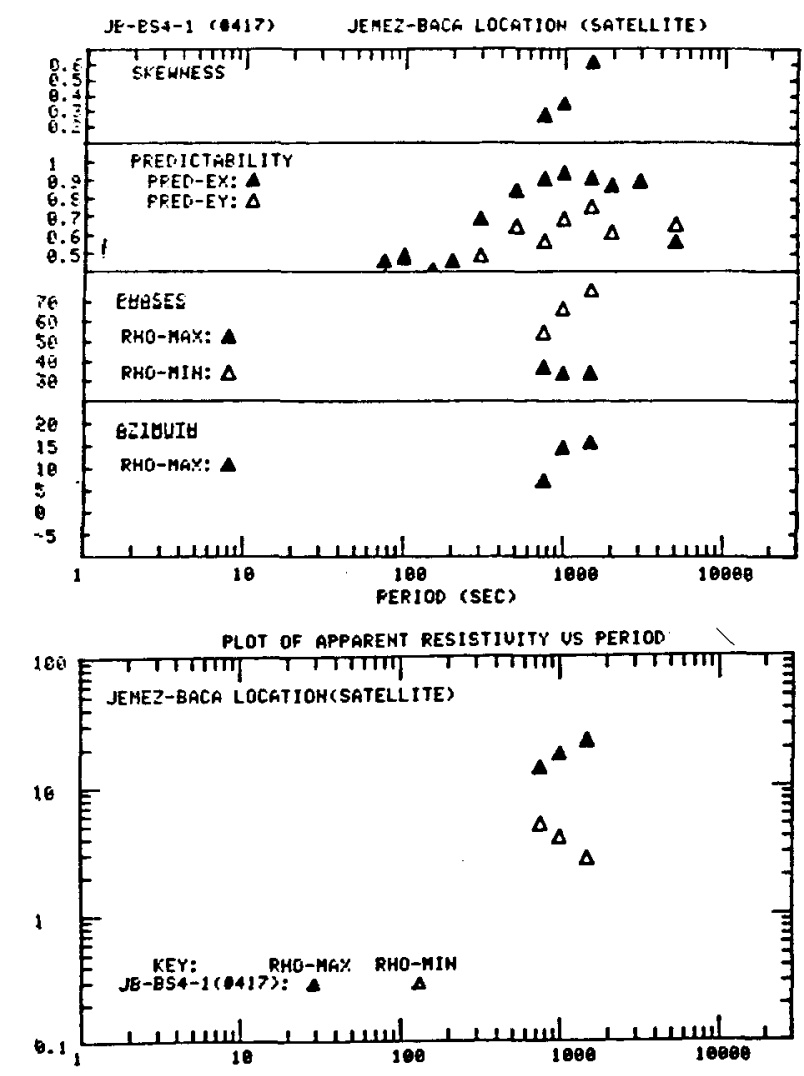